\title{
Simultaneous Wireless Information and Power Transfer (SWIPT): Recent Advances and Future Challenges
}

\author{
Tharindu D. Ponnimbaduge Perera, Student Member, IEEE, Dushantha Nalin K. Jayakody, Member, IEEE, Shree \\ K. Sharma, Member, IEEE, Symeon Chatzinotas, Senior Member, IEEE, Jun Li, Senior Member, IEEE
}

\begin{abstract}
Initial efforts on Wireless Power Transfer (WPT) have concentrated towards long-distance transmission and high power applications. Nonetheless, the less efficiency of the transmission gained and potential health concerns arising due to high power applications, have caused limitations in their further developments. Due to tremendous energy consumption growth with ever increasing connected devices, alternative wireless information and power transfer techniques have been important not only for theoretical research interests but also for the operational costs saving and the sustainable growth of wireless communications. In this regard, Radio Frequency Energy Harvesting (RF-EH) for a wireless communications system presents a new paradigm that allows wireless nodes to recharge their batteries from the RF signals instead of fixed power grids and the traditional energy sources. In this approach, the RF energy is harvested from ambient electromagnetic sources or from the sources that directionally transmit RF energy for EH purposes. Notable research activities and major advances have occurred over the last decade in this direction. In this direction, this paper provides a comprehensive survey of the state-of-art techniques, based on advances and research challenges presented by Simultaneous Wireless Information and Power Transfer (SWIPT) and WPT assisted technologies. More specifically, in contrast to the existing works, this paper identifies and provides a detailed description of various potential emerging technologies for the fifth generation (5G) communications with SWIPT/WPT. Moreover, we provide some interesting research challenges and recommendations with the objective of stimulating future research in this emerging domain.
\end{abstract}

Index Terms-RF wireless power transfer, 5G communications, simultaneous wireless information and power transfer, interference exploitation, RF energy harvesting.

This work was funded in part by the Russian Federal budget funds for research work (Fundamental research, applied research and experimenta development) through the grant No. 3942 and performed in accordance with resolutions No. 2014/226 of 2016, by the Postgraduate Scholarship of the Ministry of Science and Education, Russia, by the Shared Access Terrestrial-Satellite Backhaul Network enabled by Smart Antennas H2020 framework through the grant No. 645047, Luxembourg National Research Fund (FNR) sponsored projects on Satellite Sensor Networks for Spectrum Monitoring, Spectrum Management and Interference Mitigation in Cognitive Radio Satellite Networks, and Integrated Wireless Information and Power Networks.

Tharindu D. Ponnimbaduge Perera and Dushantha Nalin K. Jayakody are with the Department of Software Engineering, Institute of Cybernetics, National Research Tomsk Polytechnic University, Tomsk 634050, RUSSIA (email: tharindu@tpu.ru, nalin.jayakody@ieee.org). Shree K. Sharma is with the University of Western Ontario, CANADA (email:sshar323@uwo.ca). Symeon Chatzinotas is with the Interdisciplinary Centre for Security, Reliability and Trust, University of Luxembourg, LUXEMBOURG (email:Symeon.Chatzinotas@uni.lu).Jun Li is with the Nanjing University of Science and Technology, CHINA (email:jun.li@njust.edu.cn)

\section{INTRODUCTION}

The eventual fate of mobile communication is expected to be completely different from what we experience today. Ultra top-notch videos and better widescreen resolutions in mobile devices are forcing us either to look for better sustainable power sources or to perform EH from external power sources for the next-generation of wireless communication systems.

$5 \mathrm{G}$ is the proposed next telecommunication standard over the 4G/IMT-Advanced standards to meet the demands that communication networks will face by 2020 [1]. It is expected that the total number of connected devices could reach up to tens or hundreds of billions by the time that the first standard of $5 \mathrm{G}$ is expected to be matured due to many new applications in personal communication [1], [2]. As a summarization, the journey of generations of the cellular network, the first generation of communication network referred as $1 \mathrm{G}$, launched in around 1982 and it was fully analog. Second generation (2G) was launched in 1991 which used digital communication. Moreover, 2G added cellular data in the form of General Packet Radio Services (GPRS). After around 10 years, third generation (3G) networks came out introducing faster data rates than $2 \mathrm{G}$. Roughly 10 years later fourth generation communication (4G) was introduced with even faster data rates and more cutting-edge technologies. Mainly, 5G wireless communication is expected to provide an increase in data rates, bandwidth, coverage and reliability of connectivity, together with the enormous reduction in latency and energy consumption [3]. Moreover, 5G wireless networks also have integrated new communication trends such as internet of things (IoT) and machine type communications (MTC) in order to accommodate more flexible networked social information sharing one million connections per square metre [4]. The ultimate responsibility of shaping 5G has been taken by the Group Special Mobile Association (GSMA) with its partners. Development of green technology and reduction in power consumption by the devices are the two of eight major requirements of $5 \mathrm{G}$ systems identified by the industries and academia [3]. Recently, several attempts have been made to implement self-sustainable communication systems with EH techniques to support novel concepts such as IoT and 5G wireless networks, while maintaining desirable Quality of Service (QoS).

$\mathrm{EH}$ is a process of capturing and converting wasted or unimportant energy such as heat, sound and RF signals into 
electricity and putting it to work based on power requirements. The ambient environment contains high-quality energy sources compared to currently available batteries and charged supercapacitors. Lately, several studies have explored the possibility of using natural energy sources in the EH process within the communications network [5] [6]. EH using natural sources was not as effective as expected due to the irregular and unforeseeable nature of ambient sources. Also, major $\mathrm{EH}$ techniques are scenario-specific and are applicable only in specific environments [7].

WPT is one of the EH technologies that overcome the aforementioned limitations [8], where the nodes in the communications network charge their inbuilt batteries from electromagnetic radiation. Green energy can be harvested using two methods in the WPT, either from the ambient signals or from a dedicated fully controlled power source like a base station. More recent contributions based on WPT have focused on short distance (near-field) rather than long distance (farfield) energy transmission. Near-field and far-field distances depend on the application scenarios. For an example, when exchanging electromagnetic power wirelessly, the near-field distance can only be up to several meters in both indoor and outdoor environments while the far-field distance can be up to several kilometres [7]. However, near-field WPT has multiple drawbacks such as distance constraints, difficulties in maintaining field strengths within safety levels, high initial cost, difficulties in resonant inductive tuning, use of high frequency in supply and the infeasibility of air ionization techniques [9]. Within communication networks, the distance from the base station to the device is a critical factor in both information and power transfer. Therefore, far-field WPT techniques need to be further improved. The prospect of integrating WPT with communication networks creates a need for technology that can transfer both information and power simultaneously to the end-devices. For this requirement, the concept of SWIPT was first introduced in [8] from a theoretical point of view. Recently, SWIPT has attracted significant attention in wireless communication networks [10], [11]. In the era of $5 \mathrm{G}$ communication, SWIPT technology can be of fundamental importance for energy and information transmission within numerous types of modern communications networks.

With the rapid advancement of the technology, wireless transceivers and sensors are becoming more power efficient and smaller in size. We imagine as a future possibility that RF signals will not only become a power source for functional devices in the network, but their power and information transmission aspects will also be combined. Power-line communication (PLC) is a kind of communication technology that carries both information and power simultaneously through wired connections. Thus, the PLC is more appropriate for inhome applications when compared with other communication methods [12]. However, a number of technical problems exist for practical deployment of wireless PLC devices such as energy efficiency and radio interference. In order to address the aforementioned issues, SWIPT could be a promising technology towards implementing the core idea of PLC in practical scenarios. SWIPT can result in notable gains in terms of power consumption, spectral efficiency, interference management and transmission delay by enabling simultaneous transmission of both power and information [7], [8], [13]. A proper design that considers both information and power can provide noteworthy energy-efficient wireless communication and lead to possible state-of-the-art applications and services for the $5 \mathrm{G}$ and next-generation sustainable societies.

\section{A. Review of Related Survey Articles}

Only a few survey and overview articles directly related to SWIPT and EH have been published [3], [7], [11], [13], [14], [15], [16], [17], [18], [19], [20], [21], [22], [23]. A summary of these existing survey articles are listed down in TABLE I. A comprehensive survey on the next-generation of $5 \mathrm{G}$ wireless networks has been done in the work of [3] and a brief explanation about the energy requirement of $5 \mathrm{G}$ has been provided with an introduction to EH. In [7], the authors have provided a well-organized overview of SWIPT technology, including recent advances and future challenges. A rectifier antenna circuit, which is essential for the implementation of SWIPT technology, is explained in [7]. In [11], a survey is presented based on current research in radio frequency $\mathrm{EH}$ networks (RF-EHNs). It covers communication protocols, circuit designs and emerging operation designs for different types of RF-EHNs. Applications of smart antenna technologies in SWIPT enabled Multiple-Input Multiple-Output (MIMO) and relaying have been discussed in [13] along with the basics of SWIPT and receiver architectures. These works [7], [11], [13] have provided valuable insights into foundational aspects of EH and SWIPT techniques along with some recommendations. In the work of [14], the authors summarized recent advances and related results in EH and WPT enabled wireless networks from the perspective of signal processing, information and communication theory and wireless networking. Moreover, the authors in [14] have discussed a variety of topics including the effects of information theoretic physical layer performance to scheduling policies and medium access control protocols. The article [15] provided a review based on SWIPT from both the technical and theoretical perspectives. The authors have started with a discussion of different types of SWIPT scenarios including challenging issues. Then the authors of [15] surveyed various effective PHY security techniques and their impact on the performance of SWIPT. In addition, they proposed to use Massive MIMO techniques to enhance the performance of SWIPT by presenting performance gain via numerical results. Finally, a few potential future directions have been explained. A brief survey on EH and green communication in Full-Duplex Cognitive Radio networks (FDCRNs) has been done in the work of [16]. However, the main focus of the authors of [16] had been to provide an in-depth discussion of existing FD-CRN architectures and the related case studies. A comprehensive survey has been done on wireless charging compliant communication networks [17]. Moreover, the authors included existing solutions of providing WPT through static charger scheduling, mobile charger dispatch and wireless charger deployment. The latter part of [17] discussed open issues and practical challenges in wireless charging along with some future directions. The 
work of [18], provides a valuable comprehensive survey based on exploiting interference for wireless EH. The authors discussed the classification of wireless EH systems by exploiting interference based on receiver architecture, network topology, antenna dimension and interference management. Moreover, the key problems in interference management and some open research challenges have been discussed in [18]. Another survey on energy harvesting $(\mathrm{EH})$ communications and networks has been carried out in the work of [19]. Throughout [19], fundamental aspects of energy scheduling, characteristics of different energy sources, research challenges and various topics related to $\mathrm{EH}$ have been discussed. In the latter part of [19], the authors pointed out possible application systems and future directions for further development of more realistic $\mathrm{EH}$ networks. In the work of [20], the authors reviewed fundamental limits of EH communications. The authors highlighted that every transmitted symbol of an EH system is instantaneously constrained by the energy available in the battery. Moreover, partial results for the cases of infinite-sized, zero-sized and finite-sized batteries have been reviewed, while building up an awareness in the scheduling and optimization sides of the available literature. In the work of [21], the authors presented an overview and the principles of RF-EH and power transfer for wireless sensor network (WSN) applications. In [21], firstly the feasibility of $\mathrm{EH}$ and transmission has been discussed leading to the conclusion that RF EH and power transmission are more suitable for small-sized battery-powered sensors in future smart buildings. In addition, smart building integration with the RF-EH enabled sensors and the effects of RF radiation on human health were also discussed. In [22], the authors discussed the way of recent technological advancements have given rise to prominent techniques to limit energy constraints in pervasive sensor networks. The authors of [22] provided a concise review of current research on $\mathrm{EH}$ in wireless body area networks (WBANs) including potential harvestable sources and their characteristics and the usability in minimizing energy constraints. Moreover, the article [22] listed down current formidable challenges and possible future research directions in EH enabled wireless body sensor networks. While considering WSNs, a comprehensive review has been done in the work of [23] based on energy replenishment using renewable and traditional energy resources for sustainable WSNs. The work [23] also presented a survey of alternative techniques that can address the issues of limited power in wireless sensor nodes and specifically focused on EH from renewable sources as well as traditional energy resources in WSNs.

\section{B. Contributions of this Article}

Despite the existing SWIPT related articles highlighted in Section I-A, no contributions have been reported in the direction of providing a comprehensive review of the emerging technologies in SWIPT. Therefore, our objective in this paper is to focus more on SWIPT emerging technologies and its applications for next-generation communication systems. Motivated by this aspect, in this paper, we fully investigate various SWIPT emerging communication technologies with their advantages, use case scenarios, technical challenges and future directions. The scope of this survey covers RF-EH, WPT, Interference Exploitation, SWIPT, SWIPT enabled emerging technologies, key research challenges and future directions with the objective of realizing the energy-efficient 5G and beyond. The contributions of this paper are summarized as follows.

1) We provide a comprehensive summary of SWIPT and related concepts such as WPT, RF-EH and interference exploitation with comparative tables and illustrative figures. Moreover, we introduce one of the uprising topics in the domain, Interference Exploitation with a basic analysis of interference in two user link. In here our objective is to provide foundational knowledge of SWIPT, as well as some suggestions on productive areas for further research in related to SWIPT concept.

2) We survey most of the SWIPT emerging technologies from both academic and industrial perspectives based on the most recent literature of the particular emerging technologies such as such as SWIPT enabled multi-carrier systems, full-duplex SWIPT systems, bistatic scatter radio, symbol level precoding, cooperative relaying, nonorthogonal multiple access (NOMA), secure transmission, mmWave communication, WSNs, MIMO systems, multi-user Multiple-Input Single Output (MISO) and broadband wireless systems(BWS). At the end of each sub sections of emerging technologies, we have included future directions with summary and insights.

3) We anticipate the future directions of SWIPT and its applications in modern technologies such as the IoT, MTC and satellite communication. Moreover, we include recommendations on various aspects such as scheduling, hardware impairments, channel coding techniques, investigation of feedback and information theoretic frameworks to improve the efficiency of SWIPT enabled communication systems.

\section{Paper Organization}

This article is structured as follows: Section II presents an overview of RF-EH while Section III provides a comprehensive survey of WPT. Section IV explains SWIPT technology and different receiver architectures used in SWIPT. Then, we comprehensively explain interference exploitation in SWIPT enabled systems in Section V. In Section VI, we introduce emerging SWIPT scenarios. This section contains and presents well-structured explanations about SWIPT enabled emerging communication technologies. In Section VII, we focus on recommendations and future directions of SWIPT. Section VIII summarises this survey and finally, Section IX provides a table with the definitions of acronyms and notations used throughout this paper.

\section{RF ENERGY HARVESTING}

$\mathrm{EH}$ is the process of converting energy that is obtained from external energy sources such as wind, thermal, kinetic and solar energy into electricity. The environment provides a variety of energy sources, in addition to the energy stored in batteries or super capacitors. There has been considerable 
TABLE I: COMPARISON OF EXISTING SURVEY ARTICLES

\begin{tabular}{|c|c|c|c|c|c|c|}
\hline Paper & $\begin{array}{l}\text { Focused Communica- } \\
\text { tion technologies }\end{array}$ & $\begin{array}{l}\text { Focused Communi- } \\
\text { cation Generation }\end{array}$ & $\begin{array}{l}\text { RF- } \\
\text { EH }\end{array}$ & WPT & SWIPT & Key Points \\
\hline [3] & $\begin{array}{l}\text { - Massive MIMO } \\
\text { - Full-Duplex } \\
\text { - mmWave commu- } \\
\text { nication } \\
\text { - } \text { D2D communica- } \\
\text { tion } \\
\text { - M2M communica- } \\
\text { tion }\end{array}$ & $5 \mathrm{G}$ & $\checkmark$ & $x$ & $x$ & $\begin{array}{l}\text { - Existing cellular networks is- } \\
\text { sues and challenges } \\
\text { - } 5 \mathrm{G} \text { architecture } \\
\text { - Physical layer design issues } \\
\text { - Mac layer makeover } \\
\text { - Emerging applications } \\
\text { - } 5 \mathrm{G} \text { sustainable future } \\
\text { - Field tests, trials and simula- } \\
\text { tions } \\
\text { - Open issues and challenges }\end{array}$ \\
\hline [7] & $\begin{array}{l}\text { Not specific. In general } \\
\text { modern communication } \\
\text { systems. }\end{array}$ & next-generation $(5 \mathrm{G})$ & $\checkmark$ & $\checkmark$ & $\checkmark$ & $\begin{array}{l}\text { - WPT module components } \\
\text { - Wireless power receiver mod- } \\
\text { ule } \\
\text { - Conditions for efficient WPT } \\
\text { - Techniques for SWIPT } \\
\text { - Resource allocation for sys- } \\
\text { tems with SWIPT } \\
\text { - Joint information and energy } \\
\text { cooperation in CR networks } \\
\text { - Future works in SWIPT sys- } \\
\text { tems }\end{array}$ \\
\hline$[11]$ & Not specified & Not specified & $\checkmark$ & $\checkmark$ & $\checkmark$ & $\begin{array}{l}\text { - WPT Techniques } \\
\text { - Design issues in RF-EH net- } \\
\text { works } \\
\text { - Architecture of RF-EH net- } \\
\text { works } \\
\text { - RF-EH Techniques } \\
\text { - EF-EH circuit design } \\
\text { - Multi-antenna } \\
\text { networks } \\
\text { - SWIPT beamforming } \\
\text { - Multi-hop RF-EH networks } \\
\text { - RF powered CR networks } \\
\text { - Communication protocols } \\
\text { - Future directions and practical } \\
\text { challenges }\end{array}$ \\
\hline$[13]$ & $\begin{array}{l}\text { - } \text { MIMO } \\
\text { Relay assisted Co- } \\
\text { operative commu- } \\
\text { nication }\end{array}$ & Not specified & $\checkmark$ & $x$ & $\checkmark$ & $\begin{array}{l}\text { - SWIPT basic receiver struc- } \\
\text { tures } \\
\text { - MIMO SWIPT networks } \\
\text { - Relay assisted SWIPT systems } \\
\text { - The combination of MIMO } \\
\text { and Cooperative relaying in } \\
\text { SWIPT } \\
\text { - Research challenges }\end{array}$ \\
\hline
\end{tabular}




\begin{tabular}{|c|c|c|c|c|c|c|}
\hline [14] & $\begin{array}{l}\text { - WSNs } \\
\text { - Large scale WSNs }\end{array}$ & Not specified & $\checkmark$ & $\checkmark$ & $\checkmark$ & $\begin{array}{l}\text { - Information theoretic view of } \\
\text { EH } \\
\text { - Off-line energy management } \\
\text { for throughput maximization } \\
\text { - Online energy management } \\
\text { for general reward maximiza- } \\
\text { tion } \\
\text { - Medium access control for EH } \\
\text { networks } \\
\text { - SWIPT } \\
\text { - EH with large WSNs } \\
\text { - Energy consumption models }\end{array}$ \\
\hline [15] & $\begin{array}{l}\text { - Cognitive Radio } \\
\text { Networks } \\
\text { - Massive MIMO } \\
\text { - relayed communi- } \\
\text { cation systems }\end{array}$ & Not specified & $\checkmark$ & $x$ & $\checkmark$ & $\begin{array}{l}\text { - Challenges issues in SWIPT } \\
\text { - SWIPT in broadcasting chan- } \\
\text { nel } \\
\text { - SWIPT in Relaying system } \\
\text { - SWIPT in Interference net- } \\
\text { works }\end{array}$ \\
\hline [17] & $\begin{array}{l}\text { - } \mathrm{MIMO} \\
\text { - SISO }\end{array}$ & $\begin{array}{l}\text { next-generation } \\
\text { communication }\end{array}$ & $\checkmark$ & $\checkmark$ & $\checkmark$ & $\begin{array}{l}\text { - History and commercialization } \\
\text { - Fundamentals of wireless } \\
\text { charging } \\
\text { - Static wireless charging } \\
\text { scheduling strategies } \\
\text { - Mobile wireless charger dis- } \\
\text { patch strategies } \\
\text { - Wireless charger deployment } \\
\text { strategies } \\
\text { - open research issues and fu- } \\
\text { ture directions }\end{array}$ \\
\hline [18] & $\begin{array}{l}\text { - } \text { SISO } \\
\text { - } \text { MISO } \\
\text { - } \text { MIMO } \\
\text { - } \text { Massive MIMO }\end{array}$ & $5 \mathrm{G}$ & $\checkmark$ & $x$ & $\checkmark$ & $\begin{array}{l}\text { - Fundamentals of wireless EH } \\
\text { - Exploitation interference for } \\
\text { EH } \\
\text { - Interference management for } \\
\text { EH } \\
\text { - Case studies on jamming } \\
\text { based EH } \\
\text { - open research challenges }\end{array}$ \\
\hline [19] & $\begin{array}{l}\text { - } \text { Cooperative } \\
\text { networks } \\
\text { - } \text { Cognitive Radio } \\
\text { networks } \\
\text { - } \text { Multi-user } \\
\text { networks } \\
\text { - Cellular networks }\end{array}$ & $5 \mathrm{G}$ & $\checkmark$ & $x$ & $\checkmark$ & $\begin{array}{l}\text { - Energy sources and models } \\
\text { - EH and usage protocols } \\
\text { - Energy scheduling and opti- } \\
\text { mization } \\
\text { - Design issues in EH commu- } \\
\text { nication } \\
\text { - EH networking and applica- } \\
\text { tion systems } \\
\text { - Future research directions }\end{array}$ \\
\hline
\end{tabular}




\begin{tabular}{|c|c|c|c|c|c|c|}
\hline [20] & Not specified & Not specified & $\checkmark$ & $x$ & $x$ & $\begin{array}{l}\text { - Data scheduling and optimiza- } \\
\text { tion in EH communications } \\
\text { - Channel capacity with infinite } \\
\text { energy source } \\
\text { - Channel capacity with finite } \\
\text { energy source } \\
\text { - Channel capacity with zero en- } \\
\text { ergy source } \\
\text { - Noiseless binary EH channel } \\
\text { with unit energy storage } \\
\text { - Future directions }\end{array}$ \\
\hline [21] & - WSN & Not specified & $\checkmark$ & $\checkmark$ & $x$ & $\begin{array}{l}\text { - RF-EH and transport } \\
\text { - Smart building integration } \\
\text { - RF radiation and health } \\
\text { - Propagation channel } \\
\text { - Rectenna } \\
\text { - Example scenarios }\end{array}$ \\
\hline [22] & $\begin{array}{l}\text { - WSN } \\
\text { - WBAN }\end{array}$ & Not specified & $\checkmark$ & $\checkmark$ & $x$ & $\begin{array}{l}\text { - EH and WBAN } \\
\text { - Energy sources from human } \\
\text { body } \\
\text { - biochemical energy sources } \\
\text { - Ambient EH } \\
\text { - Future research directions }\end{array}$ \\
\hline [23] & - WSN & Not specified & $\checkmark$ & $\checkmark$ & $x$ & $\begin{array}{l}\text { - Energy storage techniques } \\
\text { - Renewable energy sources for } \\
\text { WSNs } \\
\text { - Energy transfer in WSNs } \\
\text { - Latest trends on the renew- } \\
\text { able and non-renewable en- } \\
\text { ergy sources in WSNs } \\
\text { - Issues, challenges and future } \\
\text { directions }\end{array}$ \\
\hline
\end{tabular}

growth of interest relating to $\mathrm{RF} \mathrm{EH}$ as the demand of WSNs, the number of radio transmitters and mobile base stations continues to increase [24]. RF energy is one form of electromagnetic energy which consists of radio waves of magnetic and electrical energy radiating through free space. RF-EH becomes a propitious solution for wireless networks with a limited lifetime. The power supply of RF-EHN has been maintained at a certain rate from RF environment built from billions of radio transmitters, mobile phones, mobile base stations and television broadcast systems around the world [25]. Therefore, the wireless devices now can harvest energy from RF signals according to the power variations over the time. In this section, we provide basic foundational knowledge on RF-EH, which helps to understand the SWIPT techniques in different emerging technologies explained in the following sections of this paper

\section{A. RF-EH Circuit Design}

The schematic of the wireless EH module in the EH devices is presented in Fig. 1 [11]. The idea of introducing this circuit design is to provide the knowledge of circuit design required to understand the features of the RF-EH enabled communication systems. Comprehensive details regarding the circuit design and electronic part of the design is beyond the scope of this paper.

1) Antenna Design: Antenna is the device in the RF-EH circuit that is responsible for capturing RF signals. Antenna efficiency is a key factor related to the operating frequency and helps to ensure the successful operation of the RF-EH system. High antenna gain while miniaturizing size are the main goals of antenna technology, which leads to a trade-off between antenna size and performance [9]. The amount of harvested energy can be dramatically increased by correctly arranging antennas with the same matching circuit and power management or operating antennas at different frequencies. RF antennas can harvest energy from a variety of sources such as mobile phones $(900-950 \mathrm{MHz})$, local area networks (2.4 GHz - 5.8 GHz), Wi-Fi signals and broadcast Ultra High Frequency (UHF) TV signals. However, this also comes with several mitigating factors such as path loss and shadowing. 
TABLE II: EXPERIMENTAL DATA OF RF-EH [26]

\begin{tabular}{|l|l|l|l|l|}
\hline Source & Source power & Frequency & Distance & $\begin{array}{l}\text { Amount of energy } \\
\text { harvested }\end{array}$ \\
\hline \hline Isotropic RF transmitter & $4 \mathrm{~W}$ & $902928 \mathrm{MHz}$ & $15 \mathrm{~m}$ & $5.5 \mu \mathrm{W}$ \\
\hline Isotropic RF transmitter & $1.78 \mathrm{~W}$ & $868 \mathrm{MHz}$ & $25 \mathrm{~m}$ & $2.3 \mu \mathrm{W}$ \\
\hline Isotropic RF transmitter & $1.78 \mathrm{~W}$ & $868 \mathrm{MHz}$ & $27 \mathrm{~m}$ & $2 \mu \mathrm{W}$ \\
\hline TX91501 Powercaster Transmitter & $3 \mathrm{~W}$ & $915 \mathrm{MHz}$ & $5 \mathrm{~m}$ & $189 \mu \mathrm{W}$ \\
\hline TX91501 Powercaster Transmitter & $3 \mathrm{~W}$ & $915 \mathrm{MHz}$ & $11 \mathrm{~m}$ & $1 \mu \mathrm{W}$ \\
\hline KING-TV tower & $960 \mathrm{~kW}$ & $674680 \mathrm{MHz}$ & $4.1 \mathrm{~km}$ & $60 \mu \mathrm{W}$ \\
\hline
\end{tabular}

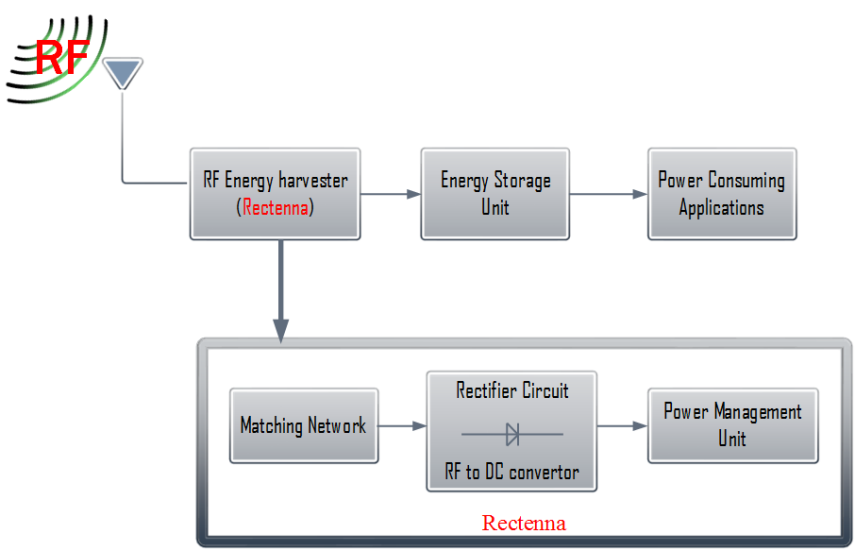

Fig. 1: The schematic diagram of the wireless energy harvesting module.

2) Matching Network: The responsibility of the matching network is to increase input voltage of the rectifier and to minimize the transmission loss from the antenna to the rectifier. When impedance of the loads and impedance at the antenna output are matching together, maximum power transfer can be achieved and is known as impedance matching.

3) Rectifier: The Main function of the rectifier is to convert captured RF signals to the DC voltage levels. One of the most important challenges of the rectifier design is to generate a considerable voltage (battery-like voltage) from the received $\mathrm{RF}$ power. RF to DC conversion efficiency is determined by the diode in the circuit of the rectifier. Therefore, the diode is recognized as the main component of the rectifier circuit. In general, a higher rectifying efficiency can be achieved by using a diode with a lower voltage [27].

\section{B. Typical Applications}

As a result of RF-EH technique, various forms of practical applications such as WSNs, wireless body networks and wireless charging systems for wireless devices have been enabled by RF-EHNs [28], [29]. In this technique, radio signals are used as channels to transfer energy in the form of electromagnetic radiation with a frequency range from $300 \mathrm{GHz}$ to a value as low as $3 \mathrm{kHz}$. Moreover, RF energy transfer can be considered as a far-field power transfer technique, as the transmitted electromagnetic signal cannot retract on the transmit antenna at a distance of above $\lambda / 2 \pi$ [30]. At the long range, RF energy can be used in different communication technologies such as D2D communication,
IoT, cooperative communication and cognitive radio networks. All these emerging communication technologies and the usecase scenarios of RF-EH are discussed in Sections V and VI. Experimental data of RF-EH from different sources are listed in TABLE II. Design issues in RF-EH networks differ for various emerging communication systems. Therefore, design issues and antenna architecture are discussed under Section IV and VI in relation to emerging technologies.

\section{Challenges}

A Few cognitive studies related to humans reviewed in [31], depicted that in the presence of RF radiation, there can be a significant delay in terms of physical response and memory reactions. Moreover, intense exposure to RF radiations can cause severe heating in biological tissues [32]. Therefore, the scientific community with the help of government agencies must gain more thorough understanding of potential health and safety impacts associated with the usage of WPT/SWIPT in public settings. Transfer distance is one of the major concerns in $\mathrm{RF} \mathrm{EH}$, but due to the inverse-square law, RF energy transfer is limited in terms of its coverage range [11]. The directivity and the gain of the receive antenna(s) critically affect RF-EH rate. Thus, developing high gain antenna which can support a wide range of frequencies also is an important research direction. Impedance matching is one of the major components in Rectennas, which determines the amount of harvested energy delivered to the rectifier. Thus, proper circuit design techniques are required, which automatically tune the parameters to reduce impedance mismatch. With the rapid advances in communication devices, RF-EH module needs to be small enough to be embedded in low-power devices. However, it is challenging to reduce the size of the RF-EH module while maintaining high EH efficiency. Further research works are required in reducing the size of the RF-EH module. The sensitivity of an information receiver is higher than the receiver used in RF-EH. Thus, the SWIPT scheme cannot be used efficiently in some of the communication scenarios. Therefore, the sensitivity of the RF-EH receiver needs to be improved in order to have an efficient SWIPT. RF powered devices have a strict power constraint and it is difficult to support algorithms that require high computational power. Therefore, all modulation techniques, channel coding, routing protocols and receiver operation policies need to be redesigned for RF powered communication networks. 


\section{Summary and Insights}

In this section, we reviewed RF-EH and the components of RF-EH module following future directions. The main idea of this section is to provide a foundational knowledge to RF-EH, which is essential to understand the content of the paper. First, we provided an introduction to RF-EH and its importance in wireless communication. Next, we reviewed the circuit design of RF-EH module and state-of-the-art circuitry implementations. Then we explained typical applications of RF-EH. Finally, we provided future directions and practical challenges in RF-EH circuit and techniques. It is shown that the EH efficiency mainly depends on the power level of received RF signal and the design of RF-EH circuit. The design of RF-EH circuit design needs to be modified according to the minimum $\mathrm{EH}$ requirement of the system and the transmission range of RF signal to improve overall efficiency of the system.

\section{WIRELESS POWER TRANSFER (WPT)}

WPT is an innovative concept that was originally devised by Nikola Tesla in the 1890s. WPT refers to the transmission of electrical energy from a power source by means of electromagnetic fields, to an electrical component or a portion of a circuit that consumes electrical power without the aid of wired interconnections. The WPT system contains a transmitter connected to the main power source, which transforms main power to a time fluctuating electromagnetic field and one or more receiver devices to receive and harvest energy from the electromagnetic field as shown in Fig. 2. Two factors, long distance transmission and high power have been studied in early attempts with WPT. Nonetheless, further development of these factors ended due to low efficiency of the power transmission process and health concerns related to high power applications [9] [10]. Therefore, most WPT research continued in two distinct regions, non-radiative (near-field) and radiative (far-field). The fields in these regions have distinct characteristics and different WPT techniques have been used to transfer the power.

\section{A. Near-Field WPT}

The near-field or non-radiative region is the area of one wavelength of the transmitting antenna. Power can be transferred by employing inductive coupling, resonant inductive coupling, capacitive coupling or air ionization (lightening) in the range of tenths of Watts [9]. In the near-field region, the WPT system requires high power transmission efficiency (PTE) and can be attained above $80 \%$ PTE [52], [9]. Power leaves the transmitter only when there is a receiver or any absorbing materials within the wavelength, which depends on the shape and size of the antenna on the transmitter. Therefore, power transmitted decreases exponentially with the distance between the transmitter and receiver, i.e., meaning if the distance between transmitter and receiver is larger than the wavelength of transmission medium, very little power can be harvested. Coupled mode theory has been used to analyse the characteristics of near-field WPT in [53] and a demonstration has been conducted by the authors using coupled antennas at the resonant frequency and $40 \%$ PTE was gained at a distance of $2 \mathrm{~m}$. It is also demonstrated in [9] that having a mediating resonant antenna in between receiving and transmitting antennas could improve PTE in WPT. Also, it is known that coupling distance and the orientation of the antennas have a big impact on optimum source and load impedance [54].

1) Inductive Coupling: In inductive coupling (inductive power transfer, IPT) used in the near-field WPT, electrical energy is transferred via coils by means of a magnetic field and high efficiency can be achieved when the coils are very close to each-other [33]. According to Ampere's law, an oscillating magnetic field has been created, once an Alternating Current (AC) passes through the transmitter coil. Once that magnetic field induces a receiving coil, it creates $\mathrm{AC}$ in the receiver. Subsequently, a rectifier in the receiver converts it to Direct Current (DC) (or drives the load directly). Few applications work at $50 / 60 \mathrm{~Hz}$, which is used in inductive coupling to recharge devices such as electric tooth-brush stands.

However, in most applications, AC current is applied only after increasing the AC frequency of an electronic oscillator, since transmission efficiency can be improved with higher frequency [34]. This technique is used in wireless charging for consumer electronic devices such as laptops, cellular phones and other portable devices, and also used to charge electric automobiles. IPT has been successfully used in Electronic Vehicle (EV) systems [35]. In early stages, charging a paddle has been used to recharge the battery of EV. However, this system is connector-less, but not wireless.

2) Resonant Circuits: Later, resonant circuits are added to enhance the inductive power transmission. Therefore, the same amount of power transferred using inductive coupling, can be now transferred at a greater distance. Nikola Tesla discovered Resonant IPT, which is one of the most well-known current WPT technologies, enabled by modern electronic components such as batteries of electric vehicles [38]. In resonant inductive power transfer (RIPT), power is transferred by magnetic fields between the transmitter and receiver resonant circuits which consist of a coil of wire attached to a capacitor or a selfresonator or a resonator with internal capacitance. There are several advantages of resonant IPT over IPT including greater range, reduced electromagnetic interference, higher frequency of operation and higher efficiency. Nonetheless, higher operating frequency in $\mathrm{kHz}$ range is one of the main advantages of resonant IPT that can be aided by the current state-ofthe art electronic technologies. Wireless power coverage is one of the possible areas for resonant IPT that can be used to power lights and recharge mobile batteries anywhere in a room without using wired connections. Another application of resonant IPT is public transit systems that supply power across the width of a roadway surface for recharging EVs batteries and to power people mover systems such as auto guided electric buses, trains etc. [39]. Wireless powering or recharging small electrical devices could drastically reduce the quantity of batteries disposed, toxic waste and ground water contamination.

3) Capacitive Coupling: Capacitive coupling is another near-field technology used to transfer power by using electric fields between electrodes like metal plates. In this technique, 


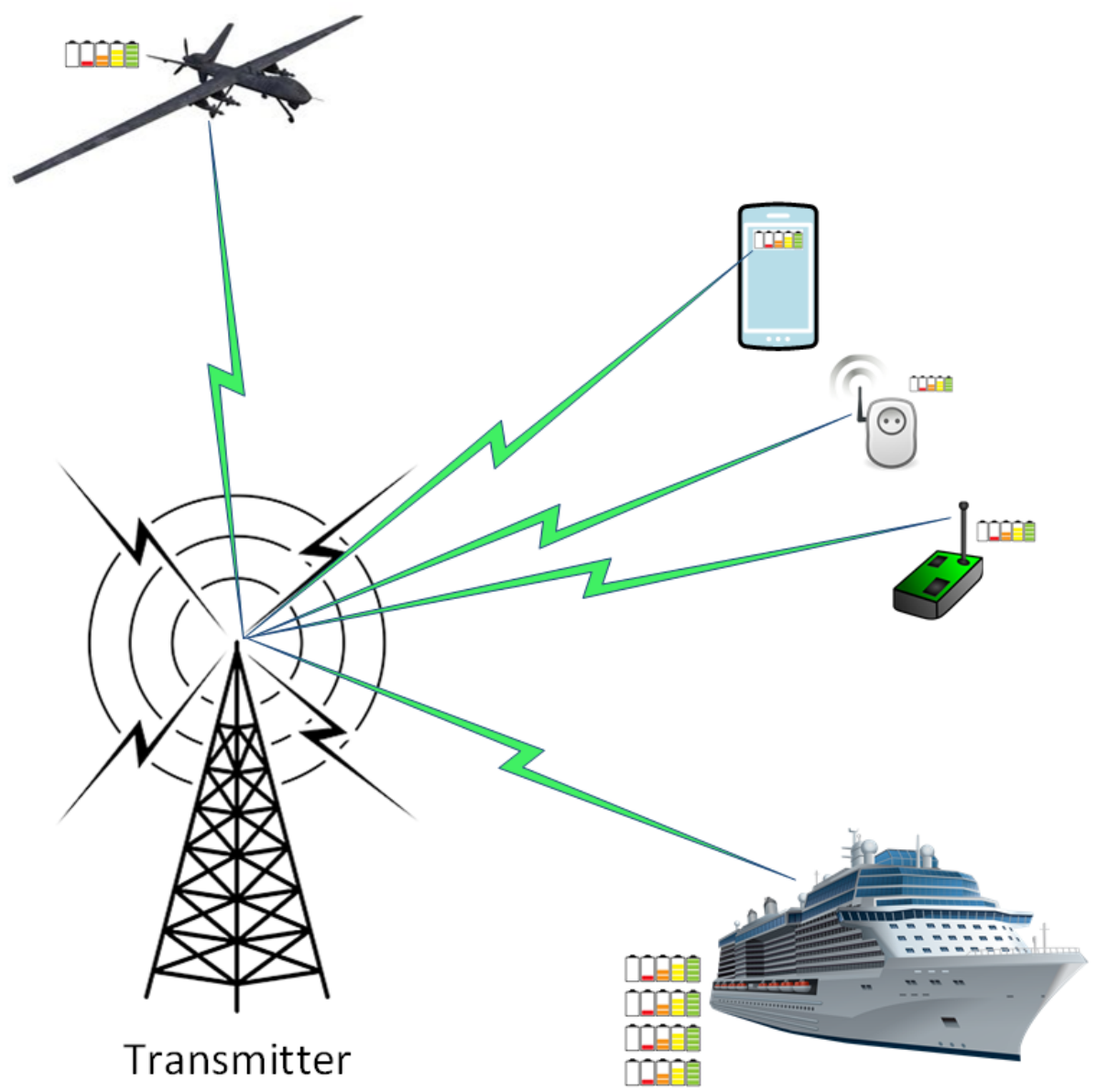

Fig. 2: RF Wireless Power Transfer - Devices such as cellular phone, sensors, drones, electronic devices in vessels receive and harvest energy from RF signals and recharge their batteries.

TABLE III: DIFFERENT TECHNOLOGIES IN WPT

\begin{tabular}{|c|c|c|c|}
\hline WPT Technology & Distance/Range & Antenna & Key References \\
\hline 6 Inductive coupling & Up to one meter (Near-Field) & Wire coils & $\begin{array}{l}{[33][34]} \\
{[36][37]}\end{array}$ \\
\hline $\begin{array}{l}\text { Resonant inductive } \\
\text { coupling }\end{array}$ & $\begin{array}{l}\text { Several meters in indoor and outdoor en- } \\
\text { vironments (Near-Field) }\end{array}$ & Tuned wire coils, resonators & $\begin{array}{l}{[38][39]} \\
{[41][42]}\end{array}$ \\
\hline Capacitive coupling & $\begin{array}{l}\text { Up to one meter - Short distance (Near- } \\
\text { Field) }\end{array}$ & Electrodes & $\begin{array}{l}{[43][44]} \\
{[46][47]}\end{array}$ \\
\hline $\begin{array}{l}\text { Microwaves, Light } \\
\text { waves }\end{array}$ & Up to several kilometers (Far-Field) & $\begin{array}{l}\text { Rectennas, Parabolic dishes, } \\
\text { Lasers, lenses, photocells }\end{array}$ & $\begin{array}{ccc}{[48]} & {[49]} & {[50]} \\
{[51]} & & \end{array}$ \\
\hline
\end{tabular}

the transmitter generated alternative voltage is applied to the transmitting metal plate and electrostatic induction causes an alternating electrostatic potential on the receiver, metal plates, which is the alternative source of current flow in the load circuit. Efficiency of the power transferred depends on the factors such as frequency, square of the voltage and the capacitance between transmitter and receiver plates [43]. Moreover, the amount of power transferred is corresponding to the area of smaller plate in the WPT system. Use of capacity coupling in high power applications can be hazardous and can produce poisonous ozone gas due to the requirement of high voltage on the electrodes to transmit significant amount of power.
Therefore, most of the time, the capacitive coupling WPT technique has only been used in applications which require comparatively low power. Nevertheless, capacitive coupling has several advantages over IPT, such as limited interference and the alignment required by both the transmitter and receiver are less critical. Recently, capacitive coupling has been applied to recharge batteries of electric portable devices and are also being used to transfer power between substrate layers of an integrated circuit. Moreover, to extend the transmitting range, resonance can be used with inductive coupling. Initial experiments with both resonant and magnetic coupling were completed by Nikola Tesla at the turn of the 20th century. 


\section{B. Far-Field WPT}

Far-field region describes the area where the distance is much larger than the diameter of the transmit antenna and was discovered immediately after the discovery of high power microwave amplifiers [48]. Far-field power transmission has enabled energy to be transferred over greater distances without using a wired electrical network and provides motivation for further research to improve the efficiency, performance and robustness of WPT applications. The most well-known forms of electromagnetic radiation used for far-field WPT are microwaves and laser beams. According to the Rayleigh criterion, over distance any kind of radio waves, such as microwaves and laser beams will become weaker [51]. Having large transmitting antenna in a WPT system compared to the radiation wavelength, can tighten the beam and spread shorter transmitting distance. When the diameter of a transmitting antenna is smaller than the wavelength, this can also cause excessive losses due to side-lobes. The microwave power beam is less vulnerable to atmospheric attenuation originated from water vapour or dust and more efficient than laser beams. Microwave power beam is proposed to apply in space applications such as energy transmission from solar power satellites to earth bases in [49]. However, practically it is difficult to use microwave power beams for most space applications due to the requirement of large aperture size. In an investigation of solar power satellites in 1978, NASA required a transmitting antenna having $1 \mathrm{~km}$ diameter [50]. However, these sizes can be reduced by having shorter wavelengths, though it can cause atmospheric absorption. Moreover, an area of $10 \mathrm{~km}$ diameter receiving array permits the use of total power levels up to 750 megawatts. These can be found in many modern electric power plants under human safe power density of $1 \mathrm{~mW} / \mathrm{cm}^{2}$. A summarized table of different WPT technologies discussed are listed in TABLE III.

\section{Challenges and Future Work}

The major challenge in WPT is the limitation on its transmission regions. Maximum energy efficiency that can be achieved in the far-field usually do not exceed 50\% [55]. There are existing systems built using microwave signals with high gain antennas, which can transfer power over far-field regions with $90 \%$ of power transmission efficiency [56], [57]. However, these systems need a point to point or line of sight connections. Nevertheless, with the advancement of communication technologies, there is a need of transferring power using omnidirectional antennas to improve the transmission region. Therefore, more future studies are needed in the far-field WPT to improve directivity and energy transmission efficiency in different communication technologies.

\section{Summary and Insights}

WPT is a concept that refers to the wireless transmission of energy from the power source to an electrical device introduced in the late 1890s by Nikola Tesla. WPT has two distinct categories, non-radiative (near-field) and radiative (far-field) based on the power transmission regions. In the near-field, power can be transmitted within an area of one wavelength of the transmitting antenna which can be up to $1 / 2$ meters. Inductive coupling, resonant inductive coupling and capacitive coupling are techniques that can be used in the near-field WPT. Far-field WPT can enable the transmission of RF power over several kilometres. Microwave and laser beams are the form of electromagnetic radiation used in the far-field power transmission. However, these techniques are only supported for point-to-point communication. Far-field techniques need considerable refinements to support omnidirectional power transmission before handshaking with modern communication technologies.

\section{SimultaneOUS INFORMATION AND POWER TRANSFER} (SWIPT)

SWIPT is a recently developed technique out of various WPT technologies and it enables the simultaneous transfer of information and power wirelessly, as shown in Fig. 3. However, fundamental design changes are required in wireless communication networks to have an efficient SWIPT. Reception reliability and information transfer rates are conventionally used to assess the performance of the wireless networks [8]. Moreover, the trade-off between information rate and harvested energy level becomes an important factor to evaluate the system performance once users in the system perform $\mathrm{EH}$ using RF signals [8]. In [8], an ideal receiver is used, which has the ability to perform Information Decoding (ID) and EH simultaneously. Two circuit receivers are used in the receiver architecture proposed in [58] to perform EH and ID separately. Furthermore, applications of smart antenna technologies in SWIPT MIMO and relaying were considered in [13]. The possibility of further improvements in WPT energy efficiency is opened up by the use of these smart antenna technologies.

In general, it is not possible to perform EH and ID operations on the same received signal in a SWIPT system, as the EH operation on the RF signal destroys the information content of the signal. Moreover, a single antenna receiver may not be able to facilitate a reliable energy supply, due to the limited collection of energy. Hence, in order to practically achieve SWIPT, either the received signal must be divided into two, or separate antennas need to be used for both EH and ID. In addition, to generate sufficient power for reliable devices, a centralized or distributed antenna array deployment (MIMO, relaying, etc.) is required [13]. In the following section, we provide an overview of several SWIPT enabled receiver architectures, including separate receiver, Time Switching (TS), Power Splitting (PS) and Antenna Switching (AS) architectures as shown in Fig. 4.

\section{A. Separate Receiver}

In this separate receiver antenna architecture, both ID and EH circuits are included as two separate receivers with separate antennas, which are served by a transmitter with multiple antennas [58]. Moreover, these two separate antennas observe different channels. This receiver architecture easily can be implemented using off-the-rack components for the $\mathrm{EH}$ and ID receivers. This architecture allows performing both $\mathrm{EH}$ and 


\section{Information Flow $\quad[-\ldots-\ldots$ Power Flow}

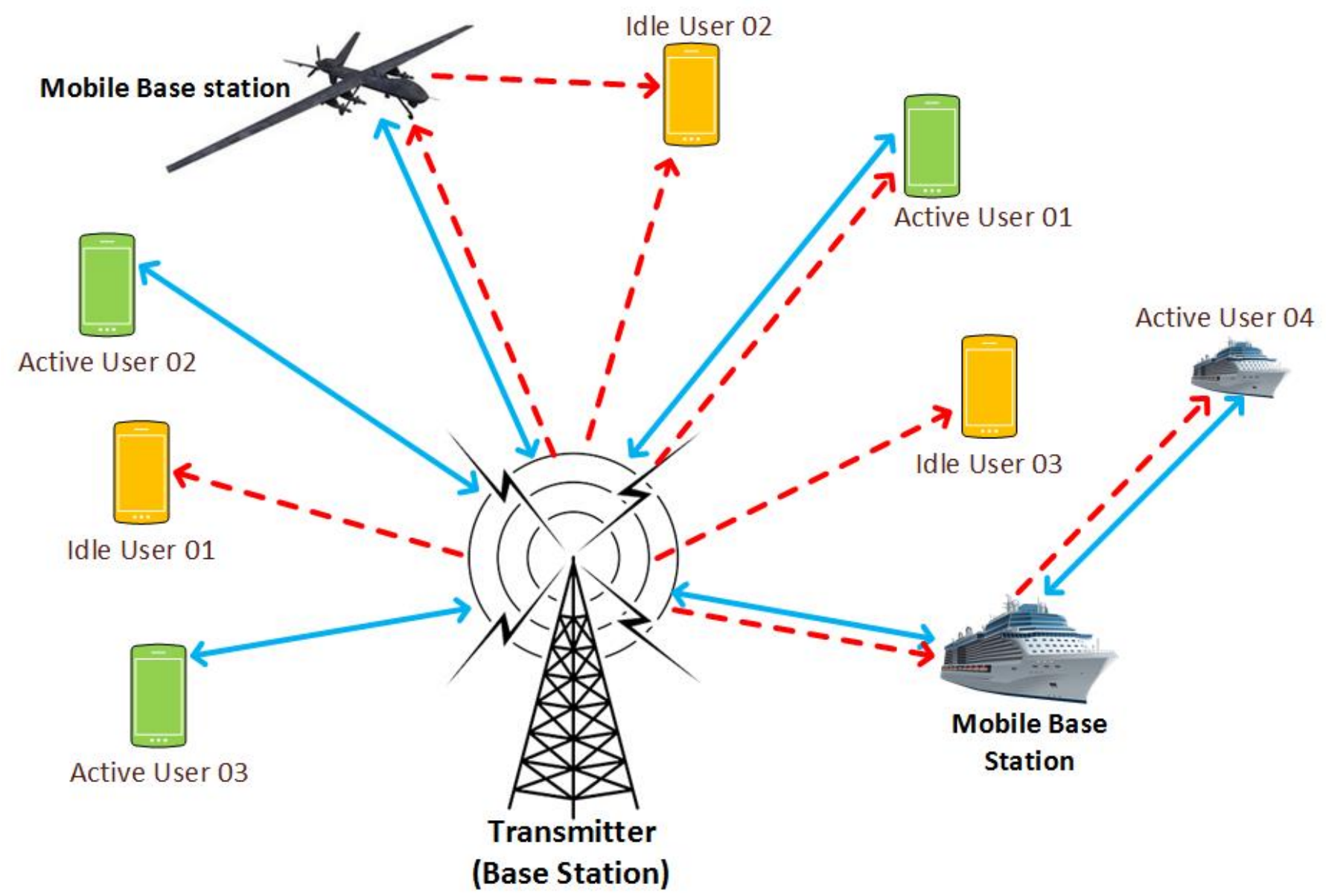

Fig. 3: SWIPT via static and mobile base stations (drones and ships). Arrow heads defines the direction of information and power flow. Idle users harvest energy from RF signals from static and mobile base stations. The active users transmit and receive information from static and mobile base station. Active users 01, 02 and the mobile base station(drone) engage in SWIPT with static and mobile base stations.

ID concurrently and independently. Channel state information (CSI) and receiver feedback can be used to optimize the tradeoff between achievable EH and information rate.

\section{B. Time Switching Receiver}

TS architecture, also known as co-located receiver architecture, share the same antenna for $\mathrm{EH}$ and for information reception. The receiver used in this architecture contains an RF energy harvester, information decoder and a switch to change the type of receiving antenna in the system. Based on a TS sequence, the receiver antenna or antennas change between ID and EH circuit periodically. Furthermore, the TS receiver also requires accurate information/energy scheduling and time synchronization. At the time receiver operates in $\mathrm{EH}$ mode, amount of harvested energy by the receiver $j$ from the source $i$ can be calculated as follows:

$$
P_{i, j}=\eta P_{i}\left[h_{i j}\right]^{2},
$$

where $\eta$ equals to the efficiency factor of EH process, $P_{i}$ denotes the transmit power at source $i$, and $h_{i j}$ represents the channel gain between source $i$ and receiver $j$. When the same receiver is changed to ID mode, ID rate can be calculated using the following equation [11], where $W$ and $\sigma^{2}$ represent the transmission bandwidth and noise power, respectively.

$$
R_{i, j}=W \log \left(1+\frac{P_{i} h_{i j}^{2}}{\sigma^{2}}\right) .
$$

Additionally, the transmit signal and TS sequence can be optimized together for different objectives of system design based on QoS requirement of energy transfer and the channel statistics.

\section{Power Splitting Receiver}

The PS receiver divides the received signal into two power streams of different power levels with a certain PS ratio before signal processing is performed at the receiver. Afterwards, both power streams are sent to an information decoder and energy harvester to make possible simultaneous ID and EH [59], [60]. Apart from the receiver circuit, no additional change 


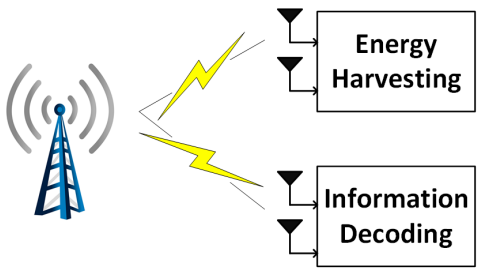

a) Separate Receiver Architecture

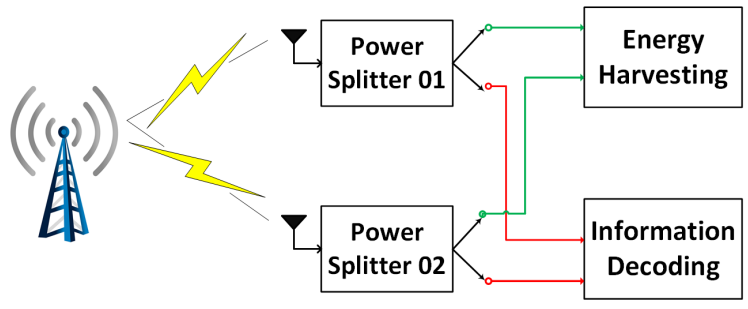

c) Power Splitting Architecture

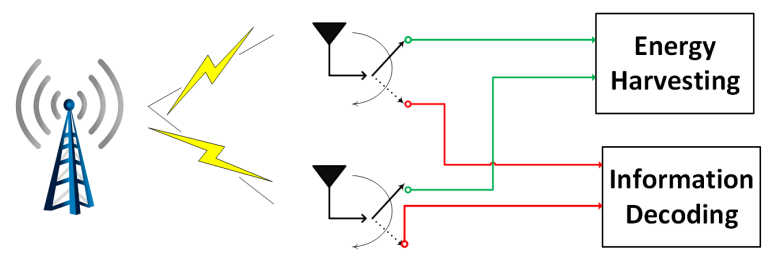

b) Time Switching Architecture
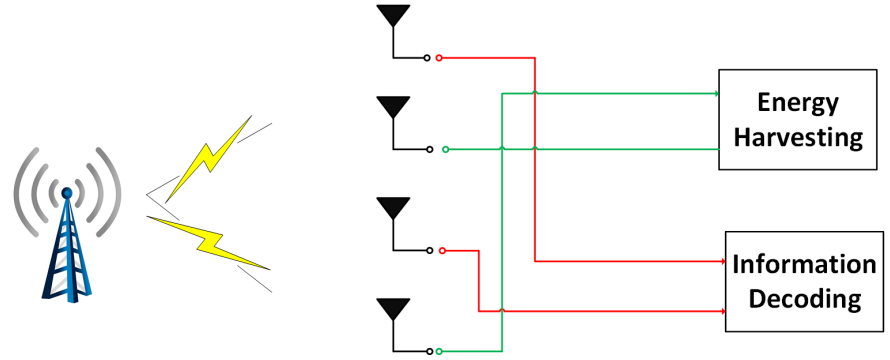

Fig. 4: Integrated receiver architecture designs for SWIPT. (a) separated receiver architecture where energy and information decoding have separated receivers. (b) Time switching architecture: based on a pre-defined time factor, connected antennas will be allocated a time to harvest energy and information decoding. (c) Power splitting (PS) architecture: the PS receiver divides the received signal into two power streams based on a certain PS ratio. (d) Antenna switching receiver: antenna switching between harvest energy and information decoding based on a optimization algorithm.

is required in the power-splitting design of conventional communication systems [61]. In each receiver antenna, the PS ratio can be optimized. Furthermore, by varying PS ratios, the information rate and the harvested energy can be balanced according to the system requirements. Overall performance also can be improved by optimizing the combination of the signal and the PS ratios. Let $\theta$ denote the power-splitting coefficient value for receivers. The amount of power harvested at the PS receiver $j$ from the source $i$ can be calculated as follows:

$$
P_{j, i}=\eta P_{i}\left|h_{i j}\right|^{2} \theta_{j}
$$

If the power of signal processing noise is denoted by $\sigma_{s p}^{2}$, the maximum ID rate of receiver $j$ decoded from the source [11] $i$ can be written as

$$
R_{j, i}=W \log \left(1+\frac{\left(1-\theta_{i}\right) P_{i} h_{i j}^{2}}{\sigma^{2}+\sigma_{s p}^{2}}\right) .
$$

Theoretically, it is recognized that power-splitting achieves the best trade-off between information rate and amount of RF energy transferred [62].

\section{Antenna Switching Receiver}

Low complexity antenna switching between EH and ID can be used to enable the SWIPT [63]. For example, while a subset of antennas at the receiver work on ID, the rest of the antennas can work on EH. Compared to time-switching and power-splitting, the antenna switching is comparatively easier and appealing for practical SWIPT architecture designs. The dual antenna receiver architecture proposed in [64] can also be adopted. In addition, with a proper antenna switching protocol, it is possible to extend this architecture easily to include a larger number of antennas. Sometimes antenna switching is also considered as a distinct case of the PS architecture [64]. Moreover, antenna switching architecture can also be used to optimize a separate receiver architecture [58].

\section{E. Summary and Insights}

In this section, we provide a brief overview of SWIPT technology along with four different receiver architectures. SWIPT is a recently introduced technology that facilitates both information and power transfer concurrently in communication networks. However, due to different sensitivity levels in both ID and EH operations, different receiver architectures are needed to facilitate the SWIPT. The received signal has to be split into two distinct parts, one for ID and the other for EH. In this section, we provided the techniques that have been proposed to achieve this signal splitting into different domains such as time, power and antenna. Summary of the existing SWIPT architectures are listed in TABLE IV. SWIPT under different emerging technologies is explained in Section VI with recent literature.

\section{INTERFERENCE EXPLOITATION IN SWIPT}

\section{A. Interference in Communication Networks}

Interference in wireless communication is anything that disrupts or modifies a transmitted signal as it transmits along 
TABLE IV: SWIPT Receiver Architectures

\begin{tabular}{|c|c|c|}
\hline SWIPT Technique & Key points & References \\
\hline Separate Receiver & $\begin{array}{l}\text { - Separate receivers for EH and ID } \\
\text { - Perform ID and EH independently and concurrently } \\
\text { - Serve by common multi-antenna transmitter } \\
\text { - Can be easily implemented } \\
\text { - Can be optimized by using Antenna Switching architecture }\end{array}$ & [58], [64], [65] \\
\hline Power Splitting & $\begin{array}{l}\text { - Required passive PS unit } \\
\text { - EH and ID perform at the same time } \\
\text { - PS ration can be optimized for each receiver } \\
\text { - Eh and ID can be balance according to requirements } \\
\text { - Achieve best trade-off between information rate and } \\
\text { amount of energy harvested compare to other techniques }\end{array}$ & [59], [60], [61], [62] \\
\hline Time Switching & $\begin{array}{l}\text { - Receiver periodically switches between EH and ID } \\
\text { - Transmitter can optimize the waveforms for EH and ID in } \\
\text { the corresponding timeslot } \\
\text { - Both ID and EH not performed at the same time } \\
\text { - Switching sequence and the transmit signal can be jointly } \\
\text { optimized for different systems }\end{array}$ & {$[66],[67],[68],[69],[70]$} \\
\hline Antenna switching & $\begin{array}{l}\text { - Low complexity antenna switching between ID and EH } \\
\text { - Performance can be degraded in case of hardware impair- } \\
\text { ments } \\
\text { - Antenna switching protocol is easy to implement } \\
\text { - Can be used to optimized separate receiver architecture }\end{array}$ & [58], [63], [64] \\
\hline
\end{tabular}

the channel between a transmitter and a receiver. In traditional wireless communication, interference is treated as a limiting factor which prevents achieving substantial quality of experience (QoE) and limits the overall system performance. Interference adds variance to the transmitted signal which deteriorates the final result [71] [72]. One of the most important design objectives of traditional communication systems is to provide low or no interference. Therefore, much great effort is needed to avoid, mitigate or cancel interference. Even though interference avoidance/mitigation techniques help to maintain effective communication between multiple users, these techniques also can cause inefficient use of available wireless resources [73]. For an instance, an effective way of utilizing wireless resources can be to allow the nodes in the network to decide on whether to mitigate or cancel interference.

\section{B. Basic analysis of Interference in a two user link}

This section provides a simple analysis of instantaneous interference in a two-user transmission scheme. A general twouser transmission link is shown in Fig. 5. We use symbol $u_{1}$ and $u_{2}$ to represent the symbols of user 01 and user 02 respectively. In this model, we use Binary Phase Shift Keys (BPSK) as the modulation technique for the simplicity, which leads to $u_{1}=1, u_{2}=-1$. Moreover, the noise at the receiver has been ignored and $\rho$ represents the interference channel and the lossless channel from the intended transmitter to the receiver. The received signal $y$ can be expressed with these basic parameters as follows [74]:

$$
y=u_{1}+u_{2} . \rho,
$$

where $u_{2} . \rho$ represents the interference. In Fig. 6, two distinct cases of interference are shown, which have the same transmit power which is equal to one. The first case gives the destructive interference where $\rho=0.5$ and it gives $y=0.5$ according to 5. Moreover, destructive interference from user 2 has shifted the received symbol of user one towards decision threshold in BPSK constellation denoted by the dotted line in Fig. 6. Thus, the received power of user 01 has been reduced and this phenomenon affects the system performance. However, in the second case, interference from the user 02 is constructive [74], with $\rho=-0.5$. From the 5, we obtain $y=1.5$. Now with the constructive interference, received power of user 01 has increased with the help of the interference from user 02 . Thus symbol detection is tolerant with constructive interference rather than in the situation without interference. 


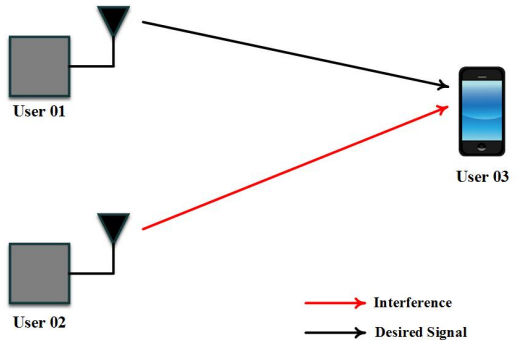

Fig. 5: A two-user link example with Interference.

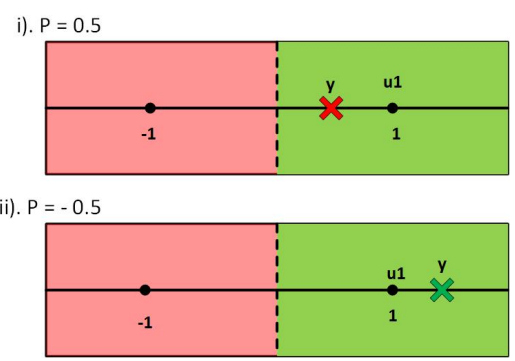

Fig. 6: Transmission scenario in two-user link with destructive and constructive interference.

\section{Interference Exploitation in SWIPT}

Recently, due to identification of new aspects of interference, instead of mitigating or avoiding, focus has been on the potential use of interference in wireless systems. Innovative ways of using interference are now emerging, since traditional mitigation techniques are no longer optimal. Thus, interference exploitation can improve the reliability, security and the achievable rate of wireless communication systems. In modern communication, interference plays a notable role in both information and power transfer in wireless communication systems [75]. SWIPT has been studied with interference broadly in multi-user systems [72], [76], [77], [78]. Although interference links are usually harmful for the ID process, they can be useful for EH. However, a proper trade-off between ID and $\mathrm{EH}$ is required to facilitate efficient SWIPT in multi-user systems. The idea of beamforming optimization has achieved an interesting level of gain by collecting useful signal power from the scenarios with constructive interference. In [79], [80], the authors proposed a multi-user symbol-level precoding approach to address the multi-user interference through jointly utilizing the CSI and Data Information (DI). The interference among the data streams is converted under certain conditions to a useful signal that potentially improves the SINR of the downlink link, resulting in improving the systems energy efficiency. Using similar set up, constructive interference has been investigated in the work [81] with the knowledge of both DI and CSI at the transmitter. Recently, some interesting beamforming strategies have been proposed to introduce a hybrid analog-digital precoding, and to exploit the concept of mutual coupling between the transmitting antennas by using tunable antenna loads [80].

In the work of [82], it has been shown that the interference does not limit the capacity of the broadcast channel. However, many works proposed Successive Interference Cancellation (SIC) instead. The employment of SIC in SWIPT enabled bipolar adhoc networks has been studied in [75]. They demonstrated how each receiver in the network can make the use of SIC to raise WPT without affecting the ID process. Moreover, results demonstrated that SIC is notably beneficial for SWIPT enabled communication systems. An Opportunistic Communication (OC) based SWIPT scheme in Interference Alignment (IA) networks has been proposed in [83]. In this work, OC based antenna and user selection are utilized to achieve SWIPT. Furthermore, this paper summarized the methods for reducing the complexity of the OC based AI algorithms. In [84], the authors investigated the constructive interference in MISO downlink to improve the performance of both the EH and ID. In addition, they introduced a new precoder design for SWIPT, which significantly reduces the transmit power and applies to the scenarios having lower number of transmit antennas than the number of users. Additionally, residual Self-Interference (SI) in multi-user MIMO channels has been studied in [85].

\section{Future Directions}

To cope up with the complexity involved in wireless communication systems, low-complexity resource allocation and optimized resource sharing are required in the future SWIPTenabled wireless networks. Therefore, interference management will continue to be a growing challenge in this domain. New perspectives on the use of interference are required to maintain the expected QoS and to meet quality and technical expectations of 5G communication. For instance, by making the use of simple linear operations to mitigate interference in future massive MIMO employed networks, interference will be available in the network to use for other activities such as EH, secure communication, etc. without degrading the overall performance of the system. This could motivate new applications and services in the next-generation wireless communications.

\section{E. Summary and Insights}

In this section, the idea of interference exploitation in SWIPT has been presented along with the recent literature. The perspective of interference has changed with the evolution of communication networks. In the present, instead of mitigating or cancelling interference, the potential use of interference in communication networks has received recent attention from the scientific community. Importance of constructive interference has been explained by using a twouser interference network. Next we provided an overview of research activities conducted in the area of interference exploitation and the context of harvesting interfering power to improve the energy efficiency of the communication system. Although the scientific community has already focused on exploiting interference in a number of different scenarios, the area of interference exploitation is quite broad having the potential in revisiting existing interference cancellation techniques in interference limited communication scenarios. In future upcoming generations of wireless communication networks, power efficiency will be a major concern and will play a major role in system design. Interference aided EH 


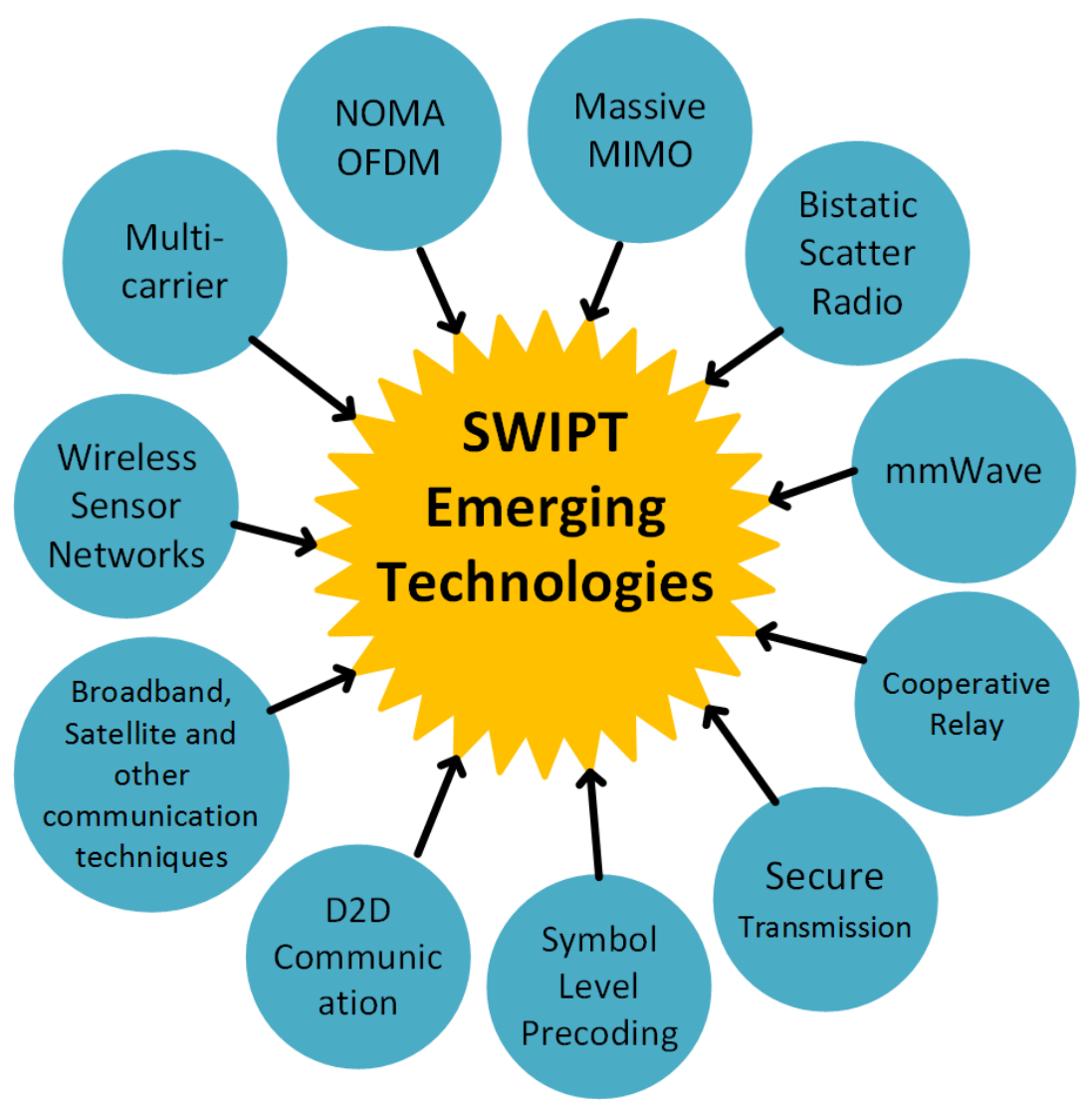

Fig. 7: Enabling SWIPT techniques for emerging wireless communication areas.

as RF power and as useful signal power can be a promising enabling solution in $5 \mathrm{G}$. In addition, the concept of constructive interference will change the perspective of the research community on the interference in communication networks.

\section{EMERGING SWIPT SCENARIOS}

In this section, we provide SWIPT enabled emerging technologies in different application areas. Fig. 7 and TABLE $\mathrm{V}$ provide various enabling SWIPT techniques for emerging wireless communication areas such as massive MIMO, bistatic scatter radio, cooperative NOMA, multi-user MIMO, BWSs, etc. All these emerging technologies are covered in the following paragraphs in conjunction with the latest literature in the field. It should be noted that, all these SWIPT emerging technologies are used in Far-Field WPT to transfer power within the communication systems.

\section{A. Multi-carrier SWIPT Systems}

Orthogonal Frequency Division Multiplexing (OFDM) is a widely used multi-carrier technique in various wireless standards. Several existing papers have studied the performance of OFDM-based SWIPT systems [86], [64], [60], [66]. The authors in [86] have investigated the performance of SWIPT receivers considering a single user OFDM channel and have provided an upper bound for the rate-energy trade-off for the considered system structure. It has been shown that there exists a trade-off between the achievable rate and the harvested energy, leading to the well known rate-energy trade-off in the considered system set-up. Furthermore, the contribution in [64] studied SWIPT for BWSs, which employ OFDM and beamforming transition in order to create a set of parallel sub-channels with the objective of simplifying the resource allocation mechanism. Subsequently, the authors proposed power control mechanisms for SWIPT in the context of a multi-user multi-antenna OFDM setting, taking circuit-power constraints into account. However, in [64], only the carriers which have been assigned to a particular user are utilized for $\mathrm{EH}$ purposes for that particular user, assuming a fixed subcarrier allocation.

Furthermore, the contribution in [60] studied the performance of SWIPT in an OFDM-based multi-user singleantenna system by employing a PS technique at the receiver. Subsequently, authors analysed the following two sets of receiver architectures:

i) the first set considered arbitrary PS ratios in order to split the received power into continuous sets of power streams.

ii) the second set considered fixed PS ratios in order to divide the received power level into a discrete set of power streams.

It has been demonstrated that system energy efficiency can be enhanced by employing RF EH in the interference limited regime. Multi-antenna receivers are beneficial to enhance the system capacity rather than enhancing the system energy efficiency [60].

Moreover, the authors in [66] have studied the performance of SWIPT systems in OFDM-based multi-user wireless 
TABLE V: EMERGING COMMUNICATION TECHNOLOGIES IN SWIPT

\begin{tabular}{|c|c|c|}
\hline Emerging SWIPT Systems & Key References & Key points \\
\hline Multi-carrier SWIPT Systems & {$[60][64][86][66]$} & $\begin{array}{l}\text { - OFDM Technique } \\
\text { - OFDM based SWIPT systems } \\
\text { - OFDM and beamforming } \\
\text { - SWIPT in multi-user, multi- } \\
\text { antenna OFDM } \\
\text { - SWIPT in multi-user, single an- } \\
\text { tenna OFDM } \\
\text { - PS and TS architectures in } \\
\text { SWIPT OFDM } \\
\text { - Multiple access schemes TDMA } \\
\text { and OFDMA }\end{array}$ \\
\hline $\begin{array}{l}\text { SWIPT enabled Cognitive Radio Net- } \\
\text { works }\end{array}$ & [11], [87], [26], [54], [88], [89], [90] & $\begin{array}{l}\text { - Architectural Design for CRN } \\
\text { - Integration of RF-EH to CRNs } \\
\text { - SWIPT enabled CRNs } \\
\text { - Main functions to support dy- } \\
\text { namic spectrum access }\end{array}$ \\
\hline Full-Duplex SWIPT Systems & $\begin{array}{l}{[91][92][93][94][95][96][67][68]} \\
{[97][98][99][100][101][102][103]} \\
{[104][105]}\end{array}$ & $\begin{array}{l}\text { - full-duplex with SWIPT } \\
\text { - Self-Interference in SWIPT en- } \\
\text { abled full-duplex } \\
\text { - full-duplex SWIPT bi-directional } \\
\text { communication } \\
\text { - TS and PS architectures in full- } \\
\text { duplex SWIPT } \\
\text { - Weight sum transmit power in } \\
\text { full-duplex } \\
\text { - weighted sum-rate maximization } \\
\text { of full-duplex } \\
\text { - full-duplex SWIPT in multi an- } \\
\text { tenna relay system and MIMO } \\
\text { - Resource allocation in full- } \\
\text { duplex SWIPT }\end{array}$ \\
\hline Bistatic Scatter Radio for EH & [106] [107] [108] & $\begin{array}{l}\text { - Scatter Radio } \\
\text { - RFID systems } \\
\text { - Path loss and limited communi- } \\
\text { cation range } \\
\text { - Integration of SWIPT }\end{array}$ \\
\hline SWIPT with Symbol Level Precoding & [109] [110] [111] [112] & $\begin{array}{l}\text { - Constructive and Destructive In- } \\
\text { terference } \\
\text { - Available precoding } \\
\text { - Channel State Information } \\
\text { - Constructive Interference as } \\
\text { source of energy } \\
\text { - Conventional SWIPT beamform- } \\
\text { ing }\end{array}$ \\
\hline Cooperative Relaying & $\begin{array}{l}{[113][114][115][116][117][118]} \\
{[119][120][121][122]}\end{array}$ & $\begin{array}{l}\text { - } \text { Cooperative relaying } \\
\text { - SWIPT to power up relays } \\
\text { - Relaying protocols } \\
\text { - Cooperative relay algorithm de- } \\
\text { sign for resource allocation in } \\
\text { SWIPT } \\
\text { - Cooperative realy algorithm de- } \\
\text { sign protocols }\end{array}$ \\
\hline
\end{tabular}




\begin{tabular}{|c|c|c|}
\hline $\begin{array}{l}\text { Non-orthogonal Multiple Access } \\
\text { (NOMA) }\end{array}$ & $\begin{array}{l}{[123][124][125][126][127][128]} \\
{[129]}\end{array}$ & $\begin{array}{l}\text { - NOMA concept } \\
\text { - Power-domain and Code-domain } \\
\text { NOMA } \\
\text { - NOMA path loss } \\
\text { - Cooperative NOMA with SWIPT } \\
\text { - Use of Self-Interference } \\
\text { - Network parameters for efficient } \\
\text { SWIPT }\end{array}$ \\
\hline Secure SWIPT Transmission & $\begin{array}{l}{[130][131][132][133][134][135]} \\
{[136][137][138][139][140]}\end{array}$ & $\begin{array}{l}\text { - QoS in SWIPT enabled commu- } \\
\text { nication systems } \\
\text { - SWIPT assisted physical layer } \\
\text { security } \\
\text { - Receiver modes of SWIPT to in- } \\
\text { crease security } \\
\text { - Secrecy performance analysis in } \\
\text { SWIPT }\end{array}$ \\
\hline $\begin{array}{l}\text { Millimeter Wave Communication } \\
(\mathrm{mmWC})\end{array}$ & $\begin{array}{l}{[78][141][142][143][144][145]} \\
{[146]}\end{array}$ & $\begin{array}{l}\text { - Use on High frequencies in com- } \\
\text { munication } \\
\text { - SWIPT enabled mmWave com- } \\
\text { munication } \\
\text { - EH schemes in SWIPT enable } \\
\text { mmWave } \\
\text { - Disadvantages of using high fre- } \\
\text { quency }\end{array}$ \\
\hline Wireless Sensor Networks (WSN) & $\begin{array}{l}{[24][28][92][147][69][148][149]} \\
{[150][151][152][153][154][155]} \\
{[156]}\end{array}$ & $\begin{array}{l}\text { - IoT } \\
\text { - SWIPT enable WSNs } \\
\text { - Classification of power usage in } \\
\text { WSNs } \\
\text { - Applying SWIPT to cooperative } \\
\text { clustered WSNs } \\
\text { - Real time wireless recharging } \\
\text { protocol }\end{array}$ \\
\hline $\begin{array}{l}\text { Multiple-Input Multiple-Output } \\
\text { (MIMO) }\end{array}$ & {$[58][70][157]$} & $\begin{array}{l}\text { - } \text { MIMO receivers/user-terminals } \\
\text { - SWIPT in MIMO } \\
\text { - Different scenarios in SWIPT en- } \\
\text { abled MIMO } \\
\text { - SWIPT with massive MIMO en- } \\
\text { abled multi-way relay networks } \\
\text { - TS and PS techniques used in } \\
\text { SWIPT enabled MIMO } \\
\text { - Secure beamforming design for } \\
\text { SWIPT enabled MIMO } \\
\text { - energy efficiency of SWIPT en- } \\
\text { abled MIMO }\end{array}$ \\
\hline A two user MISO Interference Channel & {$[58][65]$} & $\begin{array}{l}\text { - SWIPT with MISO } \\
\text { - practical schemes proposed to } \\
\text { improve EH } \\
\text { - Achievable Sum-rate with EH }\end{array}$ \\
\hline
\end{tabular}




\begin{tabular}{|c|c|c|}
\hline $\begin{array}{l}\text { Device to Device Communication } \\
\text { (D2D) }\end{array}$ & [158] [159] [160] [161] & $\begin{array}{l}\text { - } \text { Energy constraint in D2D } \\
\text { - SWIPT enabled D2D } \\
\text { - SWIPT enabled D2D in large } \\
\text { cognitive networks with new } \\
\text { WPT model }\end{array}$ \\
\hline Broadband Wireless Systems (BWS) & [64] [92] & $\begin{array}{l}\text { - SWIPT with MPT } \\
\text { - SWIPT enabled BWS } \\
\text { - Dual antenna architecture in } \\
\text { BWS }\end{array}$ \\
\hline
\end{tabular}

systems, with broadcast transmission from a fixed access point to a set of distributed user terminals. In their analysis, authors considered two multiple access schemes, namely, Time Division Multiple Access (TDMA) and Orthogonal Frequency Division Multiple Access (OFDMA). In the first scheme, the TS approach is used in such a way that the information receiver of a particular user operates in the scheduled time slot for that user, whereas the energy receiver operates in all other time periods. However, in the second approach, the PS approach is applied at each receiver, considering all subcarriers share the same PS ratio at each receiver. In the above mentioned two settings, the authors in [66] subsequently addressed the problem of weighted sum-rate maximization over all the users by adapting the time/frequency power allocation and either the PS or TS ratio considering the constraints of minimum harvested energy constraint on each user and also total and/or peak transmit power constraint.

Future Directions: Most of the existing SWIPT literature has focused on narrowband receivers for EH and ID purposes. In this context, it is interesting to investigate suitable RF EH techniques in order to harvest energy based on a wideband transmitted signal and also to explore wideband SWIPT receiver architectures. One of the promising research directions in this area is to investigate suitable techniques to enable the utilization of all the unused carriers for EH and the information bearing carrier for ID. In this case, the SWIPT receiver should be capable of working in multiple frequency bands. Moreover, it is an interesting research direction to explore RF EH from multiple sources, which are operating in different sets of frequencies simultaneously.

\section{B. WPT/SWIPT Enabled Cognitive Radio (CR)}

The basic requirement of $\mathrm{CR}$ is to be aware of surrounding radio environment and take decisions intelligently to use available resources. For example CR networks contain transmitters and receivers that can intelligently recognize idle communication channels. Moreover, CR autonomously adapts according the environment conditions by changing operational parameters such as transmit power, operating frequency, modulation and etc. [90]. This intelligent selection optimizes the use of available RF spectrum while reducing interference to other users in the network. There are two different types of $\mathrm{CR}$, spectrum-sensing based $\mathrm{CR}$ and full coordination based CR [87]. Full coordination based CR considers all attributes that a wireless network or node can be aware of during the communication process. Spectral sensing based CR is used to identify channels in the radio frequency spectrum. There are three main architectural designs for CRN; infrastructure based, ad-hoc and hybrid network [87]. Infrastructure architecture contains base station or access points through which all device communication and routing operates. There is no central entity for ad-hoc architecture and device communication using the existing communication protocols. Hybrid architecture of CRN is a combination of both infrastructure and ad-hoc architectures.

Integration of RF-EH to CRNs can provide a spectrum and energy-efficient wireless communication system [26]. In the work of [26], the authors highlighted main differences between traditional CRNs and RF-EH enabled CRNs. Then the authors conducted a study regarding trade-off among spectrum sensing, information transmission and RF-EH. The authors of [162] proposed a new CR network architecture in which secondary users harvest RF energy from the active primary transmissions. Finally, the authors have proved that this new architecture can be applied to WSN powered distributed wireless power communications.

A SWIPT enabled CRN architecture is shown in Fig. 8. The only difference in the figure compared with the general architecture of CRNs is that the secondary user participates in both information and power receiving simultaneously. As in the Fig. 8, secondary user can harvest energy while communicating with the primary base station and could possibly work as a relay in communication between the primary base station and the primary user located in the interference zone. Optimal resource allocation for harvested energy maximization in wideband CRN with SWIPT has been focused on the work of [88]. The authors of [88], initially formulated the sum of harvested energy maximization problem and then proposed a new algorithm to solve the problem and obtain the optimal power and sub-channel allocation. Cooperative access scheme for efficient SWIPT transmissions in CRNs has been proposed in [89]. The authors in [89], proposed a low-complexity multiple relay selection with gain control schemes based on compressive sensing principles.

Future Directions: Spectrum sensing, access, management and hand-off are the four main functions to support efficient and intelligent dynamic spectrum access in CR. Detecting activities of the primary user is the main function of spectrum sensing. Spectrum sensing is a challenging problem in SWIPT 

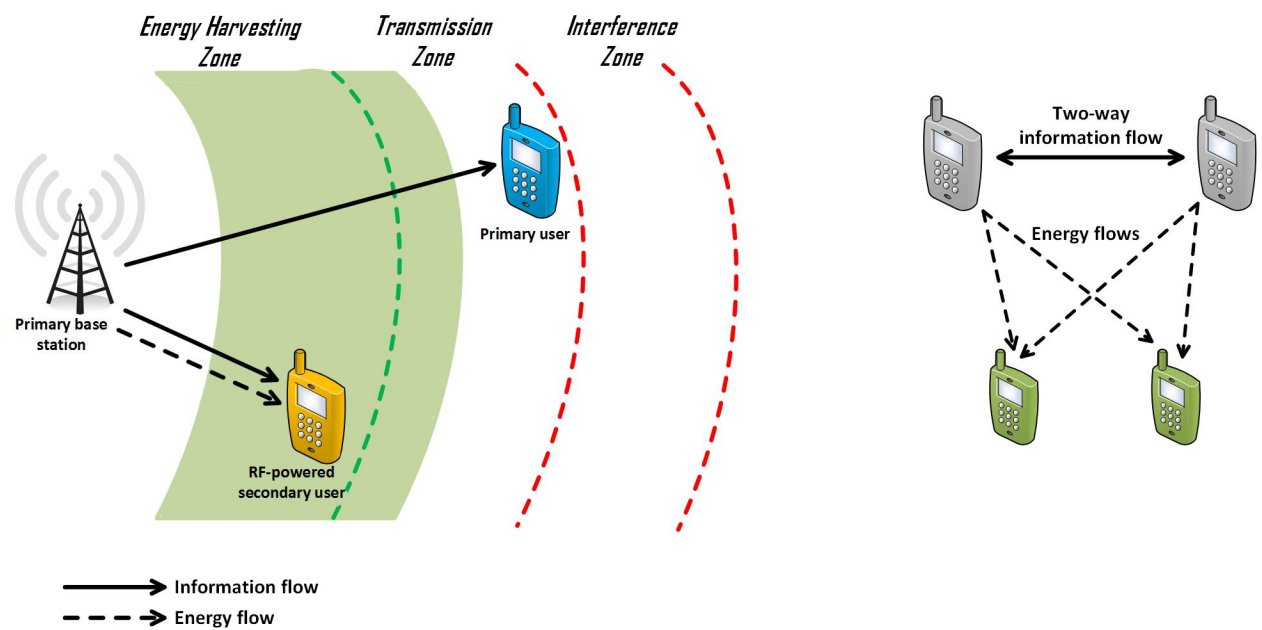

Fig. 8: A network architecture of SWIPT enabled cognitive radio network. There are three zones in around the primary base station: energy harvesting, information transmission and interference zones. Secondary user which located in EH zone can harvest energy and receive information simultaneously. Primary user in the transmission zone receive information from primary base station. Users can not transmit data if those are in the interference zone.

enabled CRNs with the complexity of received spectrum signatures from multiple devices with noise and channel impairments in the network. Furthermore, required an optimized algorithm, which could find a spectrum opportunity for both information transmission and $\mathrm{EH}$. The main issue in spectrum access in CRNs is how to provide access to available spectrum while providing fair and efficient sharing of the available spectrum while protecting the primary user from collision [11]. It is interesting to investigate the suitability of radio resource allocation during both information and power receiving. High spectrum utilization for both information transmission and RF-EH can be achieved by executing channel selection algorithms. The most valuable metrics in channel selection, channel occupancy probability and achievable EH rate need to be further investigated in SWIPT enabled CRNs. Spectrum hand-off is one of the important functionalities in SWIPT enabled CRNs. During the time the primary user re-uses or releases its channel, the secondary user has to decide whether to switch to another channel for information transmission or EH based on the availability of channels. In this direction, future research works should focus on this decision-making in order to maximize the performance of the network.

\section{SWIPT Enabled Full-Duplex communication}

In general, full-duplex communication means that both ends of the transmission link can transmit and receive signals simultaneously. Around 1940, the principle of full-duplex communication was used in radar systems [96]. Use of fullduplex communication was not encouraged until recently, due to the bottleneck of SI, which occurs due to the significant power difference between the transmitted and received signals. Due to the tremendous implications of fullduplex communication for the network design, researchers are encouraged to find solutions for SI. Enormous efforts and progress, in both academia and industry, have been made in SI Cancellation (SIC) [91], [93], [94]. SIC techniques in full-duplex transceivers are classified into two categories called active cancellation and passive suppression [95]. In passive suppression, before the SI signal is processed by the circuit of the receiver, it is suppressed in the propagation domain. Subtracting a processed copy of the transmitted signal from the received signal, the SI signal is mitigated in active cancellation. Furthermore, active cancellation can be divided into two categories based on the signal domain, digital and analogue cancellation techniques. Full-duplex communication is already recommended to be used in wireless small cells and 5G systems [96] [163]. Moreover, the application of fullduplex communication technology to physical layer security and cognitive radio systems has also been investigated [164] [165] [166]. In addition, motivation for applying CRs with the full-duplex compared with half-duplex is comprehensively explained in [16].

Combining full-duplex and SWIPT techniques will help to improve the network communication performance. In [98] [99], SI is converted into an extra source of energy in full-duplex wireless powered communication. Full-duplex SWIPT bi-directional communications, where TS or PS architecture applied to one or more nodes in the system is studied in [67], [68], [97] to perform ID and EH. In [67] with an assumption of perfect SI cancellation, the authors formulated an optimization problem to minimize the weighted sum transmit power of the full-duplex system. An antenna pair selection scheme has been produced in [68] to improve the performance of a full-duplex bi-directional system. In [97], transmit power and PS ratio were analysed with dual antenna nodes. Full-duplex relay topology was studied in [98], [163], [167], [168] with a three-node fullduplex relay channel and WPT. In [98], [99], WPT has been proposed where full-duplex nodes harvest energy through the SI channel. SWIPT with full-duplex communication has been studied in [168] with a multi antenna relying system. The source and the relay transceiver were designed according to mean-square-error criterion. In full-duplex hybrid access point topology, information transmit to users using 
uplink and received energy to users from downlink channel simultaneously was studied in [101], [102], [45], [103]. In the work of [105], authors studied throughput of full-duplex relaying in RF-EH systems with having the relays equipped with two antennas, one for information transmission and one for information reception. Moreover, it was demonstrated that using both relay antennas for $\mathrm{EH}$ during $\mathrm{EH}$ time is always beneficial and compared to half-duplex relaying architecture, the results of the [105] indicate that full-duplex relaying can improve the system throughput with optimal time split. [101], [102] investigated a hybrid full-duplex access point, where it broadcasts energy to a set of users and receives information from another set of users using the technique time division multiple access. A resource allocation algorithm was introduced in [103] for a SWIPT system which consisted for a full-duplex base station, single antenna users and multiple EH antennas. In [104], full-duplex MIMO communication network has been investigated. In that scenario, up-link users first harvest energy from the base station. After harvesting energy, up-link users transmit information to the base station, while the downlink users receive information from the base station. In certain communication networks, full-duplex shows a better throughput than the half-duplex mode. Thus, the integration of full-duplex with SWIPT can be a promising approach to improve the energy efficiency and spectral efficiency of future wireless communication networks.

Issues and Future Directions: Full-duplex communication is an enabling technology for $5 \mathrm{G}$ as it improves the throughput. Inherently, it suffers from SI issues. In order to use full-duplex incorporation with SWIPT, a robust SI cancellation algorithm enabled low-power transceiver design is needed. This creates a new direction for researchers interested in this avenue. A test bed design by incineration of non-linearity in the circuit design and suitable mathematical modelling will optimize the EH capacity of the rectifier. Interference exploitation in full-duplex needs to be studied further. Even though SI can be exploited as an extra source of energy, its capacity to improve the overall performance of SWIPT enabled fullduplex systems is unknown. Further research on transmission schemes and interference exploitation is required to balance the overall performance of the system in $\mathrm{EH}$ and information transmission. Effective combination of full-duplex-CRNs with SWIPT also can be a promising research direction. In fullduplex-CRNs, the dedicated sensing and transmission antennas require more energy and therefore, there are plenty of future research opportunities in SWIPT enabled full-duplex-CRNs to reduce energy consumption [16]. Comparatively, there is less research work done on the MAC layer aspects of fullduplex than on the physical layer. Therefore, the knowledge of MAC layer operations from an energy consumption viewpoint is not well established. Significant research efforts must be dedicated to model the behaviour of the full-duplex MAC layer. Moreover, the design of cross-layer algorithms that can utilize the information available at three layers, i.e., physical, MAC and network layers, is required.

\section{Bistatic Scatter Radio for Energy Harvesting}

A scatter radio is defined as a radio which works based on the principle of communications by means of reflected power [106]. This communication principle is commonly used in Radio Frequency Identification (RFID) systems, which are extensively being used in object tracking and supply chain monitoring. In RFID systems, a RFID tag communicates its identification number to a reader and additional information can be stored in its memory in some cases [107]. For short distances (less than 2-5 m), the tags (also called passive tags) can derive their received power from the signal received from the reader, and the communication distance is determined by the ability of the tag to extract the energy level from the received signal. However, for moderate/long distance applications, the tag (also called semi-passive tag) consists of a battery in order to operate the backscatter modulator and tag state machine and use the same backscatter mechanism for the communication purpose [107].

In the commonly used RFID systems, a carrier emitter and a reader are placed in a single reader box, which is called a monostatic RFID architecture. In other words, the transmit antenna, which generates the carrier signal and the receive antenna, which is used to demodulate the reflected signal coming from the RFID tag, are components of the same equipment [108]. However, there are several disadvantages of this monostatic architecture such as high round-trip path loss and limited communication ranges with the passive tags.

In order to address the aforementioned disadvantages of monostatic RFID structure, there is an emerging concept of bistatic architecture in which the carrier signal emitter is dislocated from the reader where backscattered signals are received. This architecture is characterized by easier setup with multiple carrier emitters and one centralized reader, and also provides long-range scatter radio communication for sensor networks [108].

Future Directions: One of the main challenges in realizing SWIPT systems is to find a reliable source for RF EH purposes. In this regard, the carrier emitters of bistatic radar systems, which are essential parts of bistatic scatter radio, can act as potential sources for RF EH. By exploiting the scatter radio emitter's transmissions, much more ambient energy can be captured. Moreover, future research work should be focused towards the development of a frequency modulation (FM) band rectifier. All FM band frequencies and scatter radio emitters frequency should be exploited to increase the acquisition and harvesting of more unused energy. Future research efforts should aim to combine the existing developed communication systems with the scatter radio sensors to design a battery-less scatter radio sensors having the FM band and emitters as a power source. Finally, an ultra large scale sensor networks should be exploited with ultra low-cost sensor nodes having capacitors as the power sources.

\section{E. SWIPT with Symbol Level Precoding}

In contrast to the traditional concept that interference is always considered to be harmful, there is an emerging concept of treating constructive interference among the users as a 
source of useful signal energy [109], [110], [111]. With the knowledge of both the instantaneous CSI and the data symbols at the Base Station (BS), the received interference can be classified as either constructive or destructive. The destructive interference deteriorates the detection performance whereas the constructive one moves the received symbols away from the decision thresholds of the constellation, thus improving the signal detection performance [110].

The available precoding for wireless downlink transmission can be broadly grouped into: (i) user-level precoding, (ii) group level precoding, and (iii) symbol level precoding [111]. The first technique is dependent on the CSI of individual users and the second technique is dependent on the CSI of each user group. On the other hand, the symbol level precoding technique, also called data-aided precoding, requires CSI knowledge as well as the symbols of the users.

Besides the possibility of using constructive interference as a source of a useful information signal, it can also be used as a source of electrical wireless energy for EH purpose [110]. For this purpose, symbol level precoding can be employed in order to exploit the constructive interference for ID and $\mathrm{EH}$ purposes. In this context, authors in [110] have recently extended the conventional SWIPT beamforming techniques in the context of symbol level precoding by exploiting the interference signal as (i) a source of electrical signal for $\mathrm{EH}$, and (ii) a source of useful information signals [112].

Future Directions: The existing literature on symbol level precoding has mostly focused on a single cell scenario. The investigation of this approach in practical multi-cell scenarios is challenging due to the need for a high level of collaboration among base stations in order to acquire the knowledge of symbols of the users. In this context, future works should focus on the feasibility of SWIPT systems with symbol level precoding in practical multi-cell systems and multiuser MIMO channels. In addition, research works also should aim towards the synchronization requirements of the system, practical impairments and the reduction of communication resource overheads.

\section{F. WPT/SWIPT on Cooperative Relaying}

In wireless networks, the nodes may not be very close to each other or may hinder each other due to several urban, climatic or geographical reasons. This results in the line of sight communication being infeasible, therefore idle nodes between them can be used to create intermediate hops as proposed in [113], [114]. However, it will be still useful to use an intermediate idle node even when a direct link in between the source and the destination is present to improve the system level performance. Hence, the relaying node can overhear the source's transmission and re-transmit its modified version of it to the destination to assist the overall decoding process [115], [116]. In the case where the relay operates using a battery assisted mode, it will not be very beneficial for the relay to participate in this cooperative transmission as it has to spend its own energy. SWIPT or WPT can stand as a potential candidate for this issue, where the source or an external access point can transfer energy to the relay
[169]. Then, the relay has an extra energy budget to help with cooperative transmission, without consuming its own energy. There are two main relaying protocols in practice, 1) decode and forward or regenerative relaying and 2) amplify and forward or transparent relaying [115], [116].

Over the past decade, interest in cooperative wireless diversity techniques has rekindled within the research community. Usage of WPT/SWIPT has also become an interesting avenue for self-powered relaying. A generic example of cooperative relay schemes is given in Fig. 9. In the first timeslot, an access point (or base station), transmits wireless RF energy and information simultaneously. The relay nodes (Relay 1 and Relay 2) are equipped with two antennas, i.e., one antenna for $\mathrm{EH}$ and other for ID. The relay harvests energy from the access point and uses it for ID and re-transmission signalling to the destination using physical layer network coding in the second time slot. Fig. 9 shows the down-link (DL) and the up-link (UL) of cooperative relay networks. Relays can also harvest energy from the RF signals of the UL transmission [170]. In this way, the relay can be a self-powered device. Such a wireless system can be powered using the presence of WiFi or using ambient signals.

Cooperative relay algorithm design for resource allocation in SWIPT systems includes the following aspects/system design protocols:-(note that some of these approaches have some overlaps though they can be categorized as below).

1) Harvest-then-Cooperate Scheme: In this scheme, authors in [117] and [118] propose a protocol related to first harvest RF energy in the relay node and then to use harvested energy for cooperation. The source and relay harvest energy from an access point or power beacon in the downlink and work cooperatively in the uplink. The resource allocation and performance measuring parameters such as throughput and energy consumption are analysed for a three node scheme in [117] and for multiple access relay scheme in [118], and in [119], it is extended to multi-antenna scenario.

2) Harvest-use scheme: In this scheme, the harvested energy cannot be stored in the battery, but should be utilised for the operational energy needs [120]. This scheme offers a trade-off between the EH time and communication time from the relay node. The fullduplex would appear to be an attractive and promising technology for harvest-use assisted cooperative relay communications.

3) Self Energy Recycling: In this technique, a part of the energy (loop energy) that is used for information transmission by the relay is used for harvesting, then reused in addition to the dedicated energy sent by the source. In this scheme, the relay can function in fullduplex mode with simultaneous $\mathrm{EH}$ and information transmission.

4) Interference Aided Harvesting: An interference aided $\mathrm{RF}$ EH operation is introduced in [121] for cooperative wireless relaying systems. In particular, energyconstrained relays harvest energy from the received information-bearing signal and co-channel interference signals. Then it uses that harvested energy to cover the 
energy expenses required to transmit the decoded signal to the destination.

Future Directions: Most of the existing literature have focused on including RF-EH and WPT to power up relays in the network. New EH scheme together with information transmission needs to be implemented by optimizing the power utilization in all relay nodes in the communication network for improving the performance of cooperative communication. Moreover, SWIPT techniques need to be integrated with aforementioned cooperative relay design protocols. Also, researchers need to focus on improving coding gain by implementing available scheme with powerful channel codes. Furthermore, the power consumption of available channel codes such as polar codes, LDPC codes and etc.. need to be investigated further in cooperative communication models. The mobility of relay nodes and its effect on $\mathrm{EH}$ also need to be studied further.

\section{G. SWIPT Enabled Cooperative Non-orthogonal Multiple Access (NOMA)}

NOMA is one of the major candidates in the design of radio access techniques for 5G [124]. In NOMA concept, there exist different solutions that can be classified into two approaches power-domain and code-domain [123].

- Power-domain NOMA - In power-domain NOMA concept, the key factor is to maintain significant differences in power levels of the signals. Therefore, it is possible to separate high level signals and cancel them out to retain a low level signal in the receiver. Hence, NOMA exploits the path loss differences in the groups of users, though it requires extra processing power in the receiver.

- Code-domain NOMA - Code-domain NOMA achieves multiplexing in the code domain. Moreover, code domain shares the available resources such as time and frequency and utilize user specific spreading sequences. There are different sub-classes of code-domain NOMA, such as low-density spreading CDMA, low-density spreading OFDM and sparse code multiple access [123].

Cooperative NOMA: Considering a downlink scheme with two user groups, the first group (near-user) enjoying good channel conditions, and the second group (far-users) experiencing poor channel conditions, applying cooperative transmission to NOMA can possibly improve the reliability of remote users. According to the proposed method in [125], neighbouring-users are used as relays in the network to improve remote-user channel conditions. The advantage of this approach is that the consecutive interference cancellation is used by the neighbouring-users and, consequently, the neighbouring-users know the information of the remote users. In [126], the use of SWIPT assisted NOMA is considered, where SWIPT was applied to the neighbouring-users, to improve the reliability of the remote users, without draining the battery life of neighbouring-users. Thus, cooperative NOMA and SWIPT communication concepts are integrated together and a new protocol called cooperative SWIPT enabled NOMA protocol is proposed [126]. It was reported that $\ln (S N R) / S N R^{2}$ is the the decay rate of the outage probability of far users while $1 / S N R^{2}$ is for conventional cooperative networks (here, $S N R$ indicates the signal-to-noise ratio). As a result of this integration (SWIPT and NOMA), careful selection of network parameters, such as transmission rate or PS coefficient can lead to an acceptable system performance using harvested energy to power the relay transmission, even without the users exploiting their own device batteries.

MISO-NOMA: Applications of MISO-NOMA systems have been investigated in [127], [128]. The work [127], studied the effect of the quasi-degradation concept on MISONOMA downlink transmission. A QoS optimization problem, considering a two user MISO-NOMA system given a pair of target interference levels was investigated in [128]. The authors of [129], investigated the performance of transmit antenna selection schemes in a two-user MISO-NOMA cooperative transmission with SWIPT enabled at near users, used hybrid TS and PS SWIPT architectures to power near user decode and forward relaying operation.

Future Directions: Most of the research works focused on power-domain NOMA with SWIPT. Therefore, more research activities are needed regarding SWIPT with code-Domain NOMA. Further research need to be done in SWIPT enabled NOMA with other communication technologies such as mmWave, MIMO and etc. In SWIPT enabled NOMA schemes, theoretical analysis is required to provide some introduction to design the system parameters according to the specific application requirements. Moreover, overall performance of the NOMA system is critically based on interference cancellation capability and complexity at the receiver. Nonetheless, these factors need to be re-analysed with SWIPT to improve overall performance of the system. Complexity of $5 \mathrm{G}$ and requirements of SWIPT need to be considered in receiver design, without increasing the complexity of the receiver. In addition, challenges in NOMA such as resource allocation, channel estimation, receiver design etc. need to be addressed before NOMA can begin handshaking with SWIPT technology.

\section{H. Secure WPT/SWIPT Transmission}

As already indicated, in SWIPT, the RF signal is used to harvest the energy at the receiver end. In order to expedite the EH process, the transmitter is able to emit a highly boosted signal. This may likewise lead to an expanded defencelessness against eavesdropping because of a higher potential for data spillage when the receiver is malevolent. In line with this, a new paradigm of QoS matter is raised in communications systems with SWIPT frameworks, which is imperative to incorporate.

In particular, privacy and authentication have progressively become major areas for wireless communications. Also, physical layer security has emerged as a new layer of defence to ensure perfect communications secrecy besides the cryptography. Cooperative relay secure transmission with PHY layer security improvements have received significant attention [131]- [132]. In [130], the secrecy rate is maximized with the help of SWIPT-enabled AF relays, limited by the EH power constraints of individual relays by jointly optimizing the cooperative beamforming relays. 


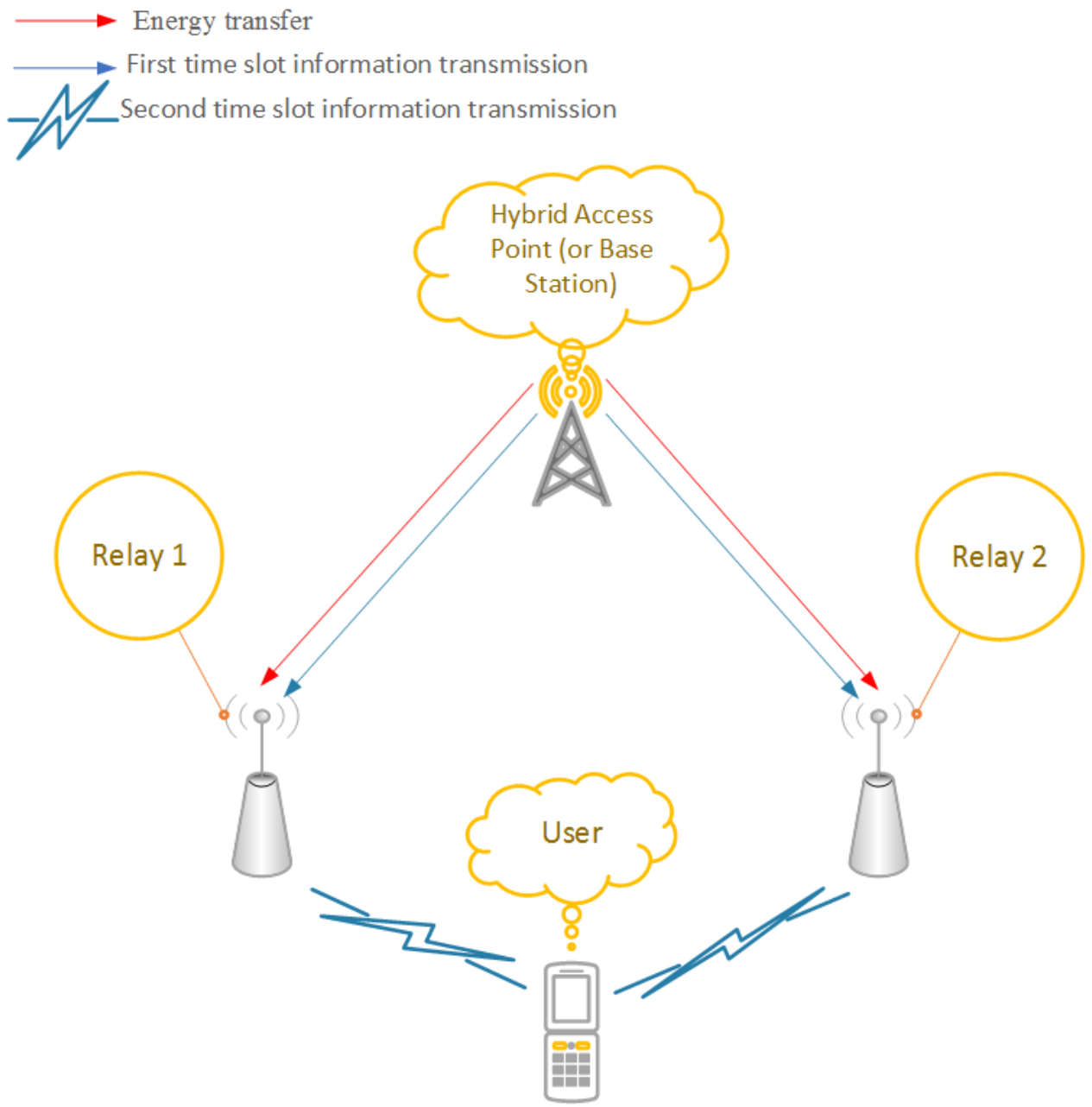

Fig. 9: An energy harvesting and information transmitting relay architecture.

By employing a separated receiver mode, i.e., one particular receiver assigned for confidential ID and the rest of the receivers assigned for $\mathrm{EH}$, some works [171] [172] [173] focus on SWIPT assisted PHY layer security. In [174]- [133], artificial noise is used to interfere with the eavesdropper for secrecy information nodes as well as to operate as the primary source of EH for non-ID receivers. This method is not suitable for co-located antennas where the user simultaneously receives secrecy ID and performs EH. In [134], [135], the authors optimize the aggregate harvested power of all users while satisfying secrecy rate requirements of individual users over an OFDMA. In [136], the secrecy performance analysis using secrecy outage and secrecy capacity metrics has been studied for single-input multiple-output SWIPT systems. In [137], SWIPT assisted secrecy issues are addressed in non-perfect CSI for the same system set-up.

Future Directions: Both power transfer efficiency and information security are equally important in SWIPT systems. These two separate objectives in SWIPT could bring new challenges for the designing phase of physical layer security in SWIPT enabled communication networks. To be more specific, both power and information signals may compete with each other for limited resources in networks and devices. Therefore, security issues and possible solutions can vary in different types of SWIPT enabled communication networks, with specific characteristics within the networks. Security issues in each and every emerging SWIPT technologies need to be further investigated with possible solutions. A proper security framework for SWIPT has not yet been developed. Since SWIPT has recently been introduced, a proper security framework or measures can uplift the possibility of integrating SWIPT with existing communication technologies.

\section{SWIPT Assisted mmWave Communications}

Low-frequency bearing signals offer less power transfer efficiency and ultimately affect the QoE of the user. As the availability of large spectrum resources at higher frequencies (above $6 \mathrm{GHz}$ ) is vital, Millimeter Wave (mmWave) communication presents as a key candidate for future $5 \mathrm{G}$ communications. We depict smart city application of mmWave enabled SWIPT based architecture in Fig. 12. In general, mmWave has been identified as a promising avenue for WPT due to the following reasons: very high frequencies, narrow beam, large array gains, and a dense network of mmWave base stations. As with the new IoT trend, many low powered connected devices are expected to deploy, and the mmWave RF signal can be used to harvest energy substantially.

Pathloss: The mmWave spectrum range is usually referred 


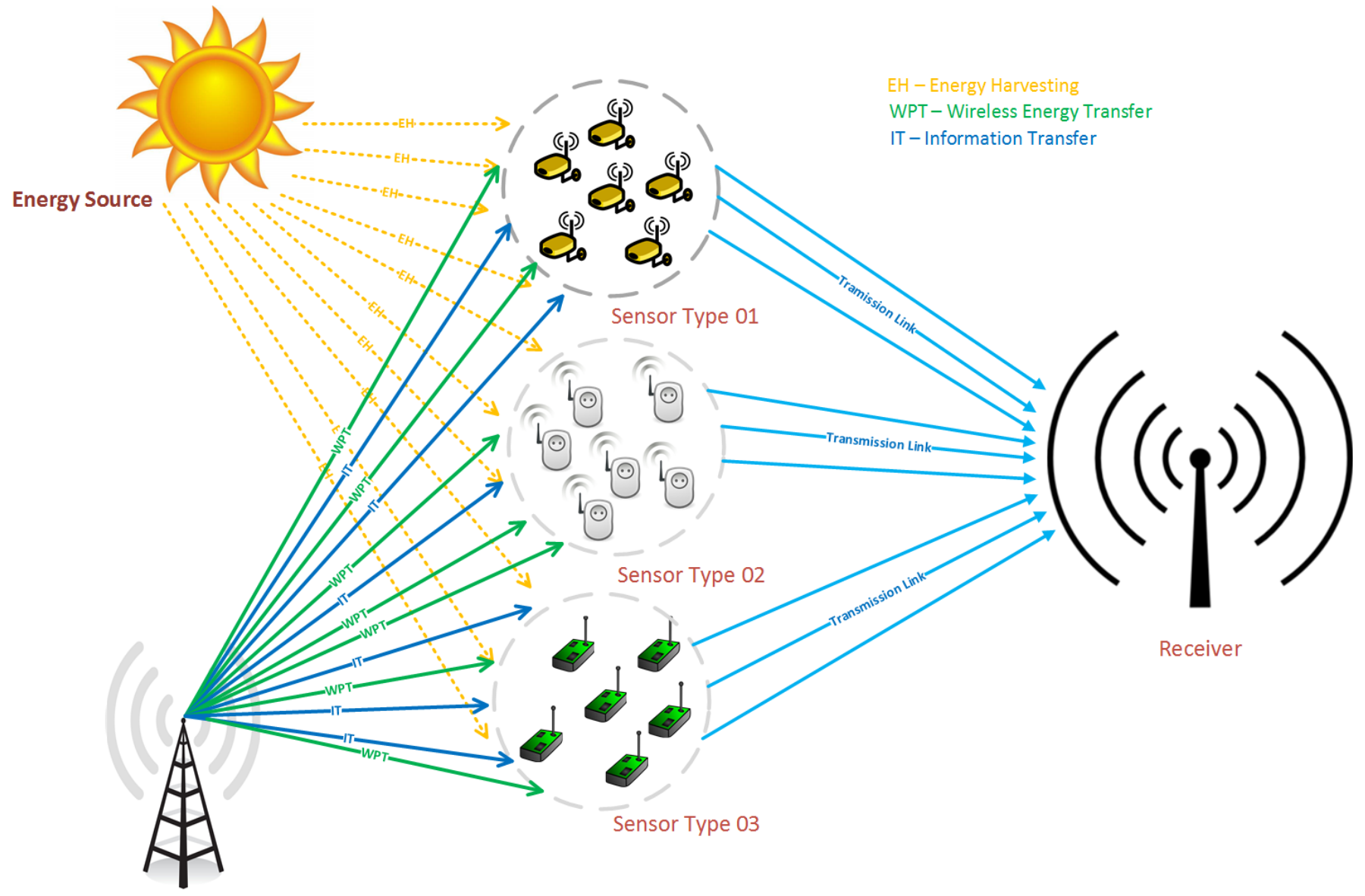

Base Station

Fig. 10: SWIPT enabled wireless sensor network system. Here we depict the dual energy harvesting policies based on the solar and RF power transfer for WSN.

between $30 \mathrm{GHz}$ and $300 \mathrm{GHz}$ since the wavelength for these frequencies is in the order of less than $10 \mathrm{~mm}$ [141]. The utilization of a higher spectrum than $6 \mathrm{GHz}$ has been reduced due to the fact that only longer wavelengths can diffract around obstacles and terrestrial terrain more smoothly [142]. However, higher frequencies including mmWaves are usually impaired with strong reflections, refractions and scattering [142]. RF propagation in the earth's atmosphere suffers from minor energy loss due to the atmospheric absorption [143]. In general, mmWave spectrum signals undergo higher atmospheric absorption than the signals using lower frequencies [142]. Nevertheless, for short distance communication, atmospheric and rain absorption are not limiting factors in mmWave spectrum since around 250 meters these absorptions are as less as $4 \mathrm{~dB}$ in $61 \mathrm{GHZ}$ links and $0.5 \mathrm{~dB}$ in $24 \mathrm{GHz}$ [142].

An EH scheme demonstrating harvest and use strategy is applied in [144] where the user harvests energy from the corresponding base station through mmWave signals, then uses the harvested energy to transmit information messages. The overall (energy-and-information) coverage probability is improved by use of an optimizing technique over the PS ratio in order to receive a signal (optimally portion) between the $\mathrm{EH}$ and the ID modules using mmWave assisted SWIPT in [78]. Here, the authors maximize the network-wide energy coverage for a given user population by optimizing the antenna geometry. In [145], a prototype is developed for 24 $\mathrm{GHz}$ rectifying antenna. This demonstrates the feasibility of wireless $\mathrm{EH}$ and information transmission by the use of the Millimeter-wave. Moreover, an additional advantage of the mmWave spectrum is that high antenna directivity can be achieved using small form factor printed antennas [146]. This will allow the mitigation of interference, which can be used in SWIPT as an extra source of energy. In addition, transmitter will focus the entire power towards the intended receiver, which also can improve EH efficiency in SWIPT assisted mmWave communication.

Future Directions: Unfortunately, as high-frequency bands inherently suffer from poor penetration and diffraction, they present as research challenges in using mmWave for WPCN and SWIPT enabled wireless networks. Also, a limited amount of work has considered mmWave for SWIPT/WPCN. There is a huge gap in the research on mmWave assisted SWIPT in information processing and hardware development. Many of current setups cannot be used directly for higher frequencies, i.e., above $6 \mathrm{GHz}$ due to the path loss and health concerns. Further research activities needed in modelling path loss problem in SWIPT assisted mmWave communication. 


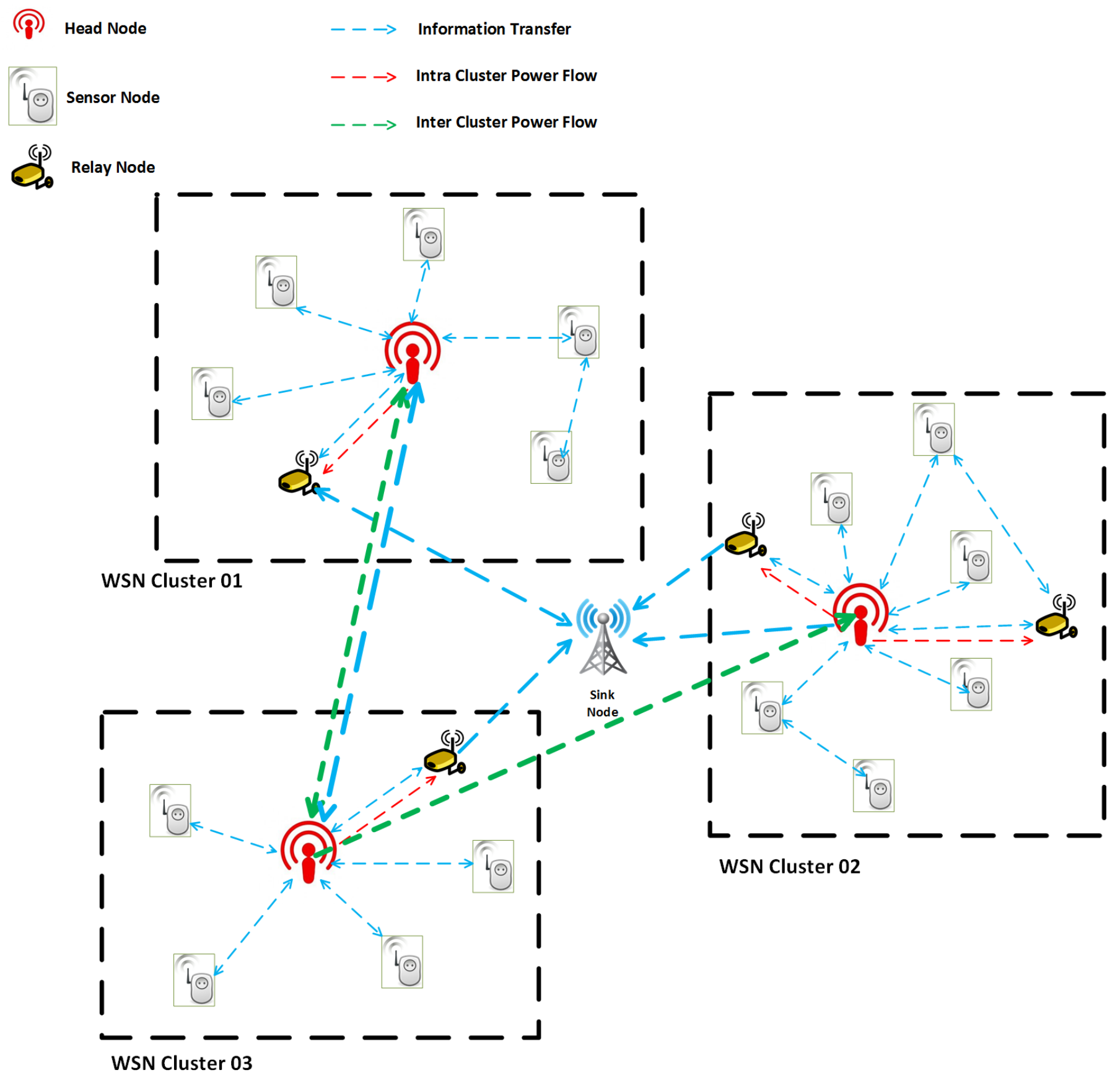

Fig. 11: A clustered SWIPT enabled wireless sensor network consisting multiple clusters of sensors and a sink node which is responsible for collecting data from each sensor. The head node works as both an information and power transferor while relay node receiver harvest the RF energy from the head node of the cluster and recharge the batteries.

\section{J. SWIPT/WPT Enabled Wireless Sensor Networks}

The WSN has come to significant attention with the new embarkation of the IoT. Some of the devices are small in nature but, generally, placed in hazardous or remote areas where human access is limited. Therefore, replacing the batteries or supplying a stable power source is an issue for WSNs. SWIPT enabled WSN is shown in Fig. 10. In [147], the authors have proposed a hybrid TS and PS spectrum sharing protocol for EH wireless sensor nodes. In WSNs, power usage is usually divided into three parts as in [69]: sensing, data processing, and communication. As compared to sensing and data processing, communication is a costly functionality in a typical sensor node [148]. Hence, local data processing is important in order to minimize power utilization of WSNs. As the failure of any node can significantly cause the structure to be re-routed, causing inefficiency in terms of reliability improving the lifespan of WSNs are important stems for future IoT.

\section{Clustered WSNs with SWIPT:}

Applying SWIPT to re-charge low energy relay nodes in cooperative clustered WSNs has been considered in [175]. 


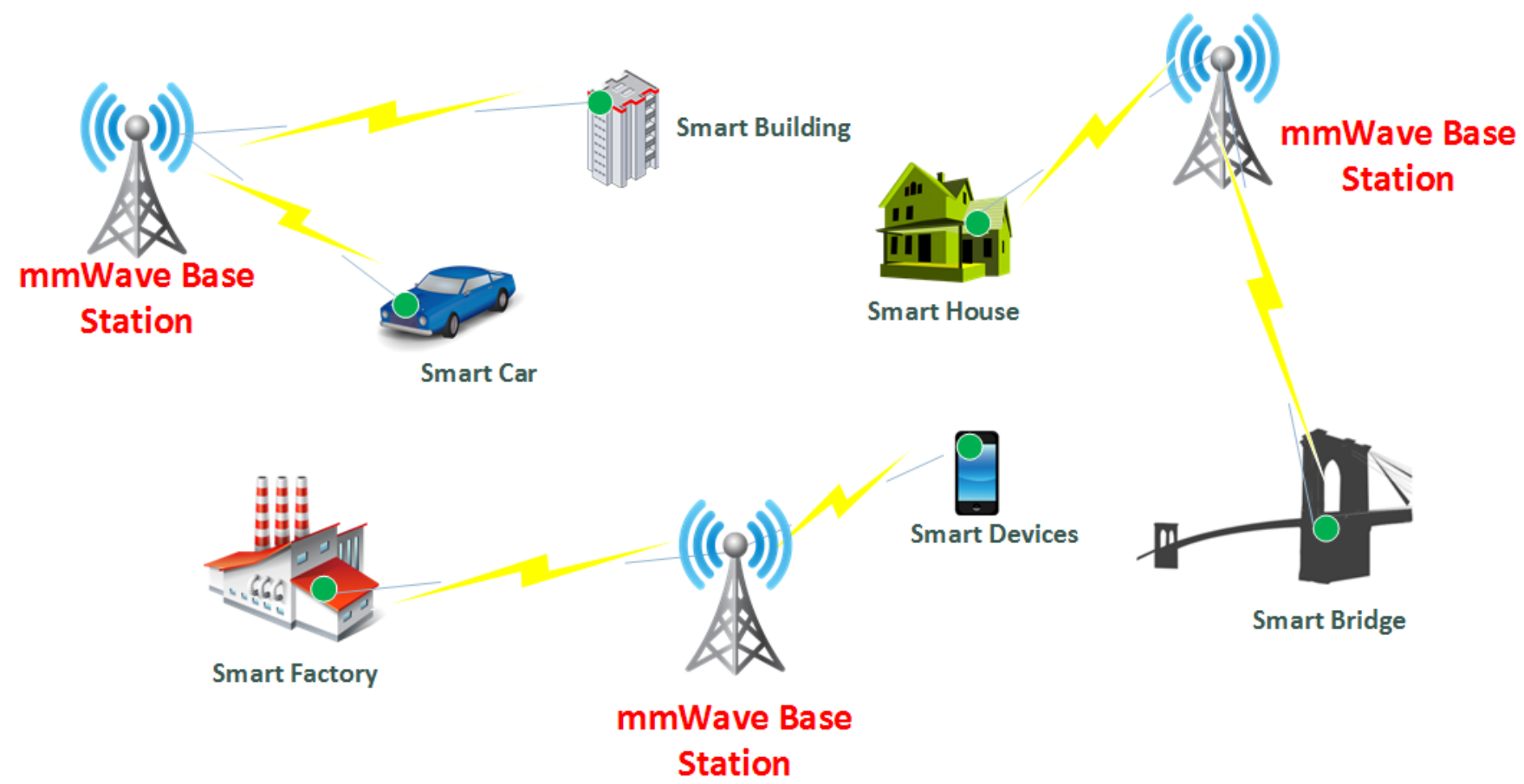

Fig. 12: An mmWave enabled wireless power transfer scheme for smart city.

As shown in Fig. 11, they consider a WSN consisting of multiple clusters of sensors and a sink node responsible for collecting data from sensors in clusters. In this scenario to enable SWIPT, the head node of each cluster works as both an information and power transferrer. Relay node receiver harvests the RF energy from the head node of the cluster and recharges the batteries. Furthermore, authors of [175] proposed a distributed iteration algorithm with closedform transmission power, PS ratio and relay selection by utilizing decomposition. In [149], the authors focused on the development of network deployment and its routing strategy. The idea is to reduce the total recharging cost to enhance the lifespan of WSNs. An optimization problem is formulated using joint network deployment and routing in [24] by having an assumption that the sensors can be continuously recharged before their power source is exhausted and have complete knowledge of CSI. A few prototype implementations of sensor nodes using RF EH are investigated in [24], [150]. By reaping the benefits offered through SWIPT, low power WSNs deployed in the health care sector can recharge power sources while they are in operation and this certainly improves the QoE [28].

Some works have suggested the use of a wireless energy charger application for WSNs, as it supports mobility [151], [152]. A practical real time wireless recharging protocol for dynamic wireless recharging in sensor networks is proposed in [153]. The real-time recharging framework supports single or multiple mobile vehicles. Similarly, a collaborative wireless charging scheme has been presented in [154]. Recharging selected nodes in remote area with Unmanned Aerial Vehicles (UAVs) delivers a solution that presently can recharge a single node. In [155], the authors simulated a limited recharging system and presented the evidence of the effectiveness of recharging a single node. However, this does not describe a robust system model and work is limited to simulations. Prototype based implementation for such EH architecture is provided in [156].

Future Directions: A substantial amount of work concentrates on the aspect of wireless EH and relatively less work concentrates on SWIPT enabled WSNs. Interference exploitation based EH techniques have not yet been largely investigated. Physical layer redesign of SWIPT enabled WSNs is a timely requirement to advance research within a solid theoretical framework. More research activities need to be carried out in selecting proper energy storage mechanism since it is directly related to efficiency and long operational life time of sensors. Robust communication protocols are also vital to efficiently exploit energy sources and must be proposed to offer an ultimate self-sustainable solution.

\section{K. SWIPT Enabled MIMO systems}

MIMO is an antenna technology for wireless communication which has been comprehensively researched during the last two decades. It is used in numerous wireless networks, since it can notably improve the reliability and capacity of wireless networks. Initial research works focused on point to point MIMO links which use two devices with multiple antennas to communicate with each other. Now focus has shifted to multi-user MIMO systems, where the base station having multiple antennas concurrently serves a group of single-antenna users [70].

MIMO SWIPT: In the MIMO network, all receivers/user terminals are battery limited devices, and batteries need to be recharged to extend the network lifetime. EH is performed by 
the individual receivers through dedicated power transmission from the transmitters in the network. Most of the work that tried to integrate SWIPT in MIMO wireless networks assumes that there are two defined groups of users to be served, one for receiving information and another for receiving power to recharge their power sources. To have a better understanding, a three-device (one transmitter and two receivers) MIMO wireless broadcast system is considered as shown in Fig. 13. Using the signal sent by a transmitter, the energy receiver harvests energy while the other receiver performs ID. Two different scenarios in the MIMO broadcast system have been investigated in [70], namely separate and co-located ID and $\mathrm{EH}$ receivers. In the first scenario (separate receivers), the best transmission strategy was designed to attain non-identical trade-offs in SWIPT. Those trade-offs are characterized by the boundary of the rate-energy region. For the second scenario (co-located receivers), the previous solution can also be applied to the unique MIMO channel from the transmitter to the EH and ID receivers. Furthermore, two SWIPT techniques, TS and power switching, were investigated for the case of co-located receivers [70].

Massive MIMO SWIPT: Massive MIMO is a scaled version of MIMO technology containing thousands of antennas and terminals. Massive MIMO provides the new extent of efficiency and throughput by the antennas attached to the base station, focusing the reception and transmission of signal energy into a small region of space. Moreover, partial focussing also can be improved with the increase of antennas. In the seminal work of [58], SWIPT in massive MIMO enabled multi-way relay networks (MWRNs) and energy constraining amplify-and-forward relays have been investigated by utilizing TS and PS techniques. Once the quantity of relay antennas grows in the network, sum rate expressions and harvested energy have been derived from a considerable amount. The effect of co-channel interference (CCI) on the performance metric was also investigated in [157], and the result proved that the CCI can be utilized in the relay for improving EH. Integration of SWIPT and MWRNs with massive MIMO can be a significantly improved trade-off between the performance of energy constraint AF relaying and the harvested energy. The use of SWIPT in Massive MIMO enabled wireless networks has a potential to attain a substantial improvement in energy efficiency. Furthermore, it also works well with many applications associated with millimetre wave communications and heterogeneous networks. In [176], the authors have investigated secure beamforming design for two user MIMO broadcast system for both information and energy transmission. For the design, authors have proposed semidefinite relaxation based best suitable solutions, for both the single and full stream cases with channels satisfying positive semidefiniteness. Meanwhile, with an arbitrary number of streams, the inexact block coordinate descent (IBCD) algorithm has been proposed for the general case. According to [176], extension of this work can be the optimization of the IBDC algorithm to more scenarios in secure beamforming with multiple information and energy receivers.

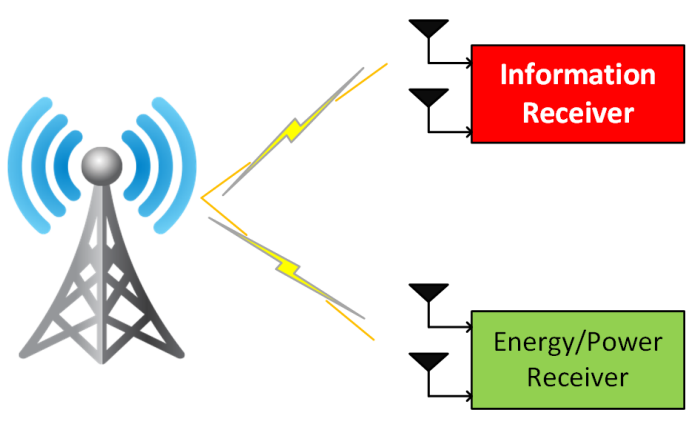

Fig. 13: A basic MIMO broadcast system. Here a set of antennas are allocated for the information decoding and another set is assigned for the RF-EH.

Future Directions: Due to the path loss, signal strength is reduced by a significant amount. Therefore, the energy efficiency of MIMO SWIPT systems might not be as high as expected for long distance power transfer. To overcome this bottleneck, more research works are needed with integration of advanced green EH technologies with MIMO resource allocation. In addition, more research activities need to be focused on communication security in MIMO SWIPT due to the vulnerability of information leakage.

\section{SWIPT: A two user MISO Interference Channel}

A single antenna is used at both source and destination in typical wireless communication networks, which causes issues in the communication link with multipath effects. Issues caused by multipath wave propagation can be reduced by MISO technology, having two or more antennas with the transmission of multiple signals at the source. In MISO, the finest optimal transmission strategy for the best scenario has been studied where the two receivers in the communication system simultaneously harvest energy and decode information [65] to implement self-sustained wireless communication networks. It is also worthwhile to note that cross-link signals are useful in improving $\mathrm{EH}$ of the receivers in spite of the fact that it limits the achievable sum rate [65]. Thereafter, considering the current limitation of circuit technology, two practical schemes are proposed based on TDMA, where the receiver performs ID or $\mathrm{EH}$ at each time slot.

1) The first scheme named $A$, splits each transmission interval into two time slots, in which one slot is used to perform EH by both receivers and afterwards to perform ID in the next time slot.

2) The transmission time of the second scheme, named as $\mathrm{B}$, divides into two time slots, as in scheme $\mathrm{A}$, with the difference that, in each time slot, one receiver performs ID whereas the other receiver performs $\mathrm{EH}$ operation simultaneously.

The achievable sum rate of these proposed TDMA schemes $\mathrm{A}$ and $\mathrm{B}$ in ideal conditions was studied via simulations [65]. It was proved that the ideal scheme, which uses ideal receivers, is sometimes not the best proposition with reference to sum rate maximization. In an interference limited system, TDMA of scheme A offers a better sum rate as compared to the ideal scheme. When one of the receivers requires 
comparatively higher energy than the other, TDMA of scheme B provides a higher sum rate than TDMA of Scheme A. Overall simulation results and the conclusion are very different from the previously reported results [58] in the absence of the ideal receiver, which always performs better.

\section{SWIPT Assisted Device-to-Device Communication}

Device-to-Device (D2D) communication provides direct communication between the mobile users without or partial network-infrastructure utilization. D2D communication improves network throughput, spectrum efficiency and energyefficiency, while supporting various location-based and peerto-peer applications and services. It plays an important role in improving the network capacity and resource utilization [158]. An additional feature of this technology is that the coverage can be significantly improved by the use of a relay node. The relay node enhances the D2D network performance, while a mobile user equipment (UE) communicates with the other user. Relay user equipment relay (UER) node works as an intermediate node [158], [159].

Due to energy constraint in transmitters, integration of SWIPT with D2D communication is identified as a progressive research area. Availability of sufficient energy in devices is important to ensure the power demands of D2D applications. This approach became ineffective due to the use of UERs own power for data transmissions of other UEs . As a solution for this bottleneck in [160], a D2D communication enabled EH cellular network where the UER harvests energy from a base station and uses it for D2D communication is considered. Moreover, SWIPT enabled D2D communication in large scale cognitive networks has been studied in [161]. They proposed a new WPT model with battery free design at the energy constraint D2D transmitter. In addition, when processing the WPT, the impact of small scale fading is also considered in this proposed model. SWIPT assisted D2D communication network is shown in Fig. 14.

Future Directions: A limited amount of research has been conducted on SWIPT enabled D2D communication. Integration of D2D communication with the WPCN would be a major advance towards achieving SWIPT in D2D communication. Several issues such as power control, resource allocation, relay node association, relay node selection and management, mode switching, etc. will need to be addressed in order to improve the power transfer efficiency of SWIPT enabled D2D communication networks. We predict that this will evolve into one of the key technologies in the $5 \mathrm{G}$ communication for the years to come.

\section{N. SWIPT Enabled Broadband Wireless Systems}

A wireless broadband is a wireless network technology that addresses the last mile major problem in telecommunications. It provides high-speed internet and data services via a wireless local area or a wide area network (WLAN or WWAN). The local multipoint distribution system and the multichannel multipoint distribution service are fixed wireless broadband technologies for broadband microwave wireless transmission heading from a local antenna to the destinations. Integration of SWIPT with Microwave Power Transfer (MPT) within a broadband wireless system is a promising technique for the need of convenient energy supply in wireless networks [64]. The broadband wireless system with limited interference contains multi-antenna base stations for information transfer and power transfer to free the wireless devices from the limitation of restricted battery capacities. Far-field MTP has been used to support SWIPT with the understanding of the QoS. In this approach, using orthogonal OFDM, the broadband channel is split into multiple orthogonal sub-channels, where the base station in the communication network transmits/receives one data stream per sub-channel created. Depending on the transmission policy, each stream is encoded at a fixed rate or with one of the variable rates, according to the reported received SNRs. It is noted that without beamforming, OFDM alone can decouple only an information transfer link, not an MPT link. Based on OFDMA, this work considers both single-user and multi-user systems, where the mobile device is assigned either to all sub-channels or to a single subchannel. Depending on the direction of information transfer, downlink or the uplink, two practical courses for SWIPT are considered. In the first scenario, SWIPT with downlink information transfer uses an OFDM signal which is transmitted by the base station in the broadband wireless network, for both information and power transfer. The second approach deals with uplink information transfer, where information and power transfer proceeds in opposite directions. Downlink MPT depends on the transmission of power tones categorized as unmodulated, while uplink data signals are OFDM modulated.

SWIPT enabled BWS contains dual antenna to support the architecture in Fig. 16, which can be reconfigured depending on the direction of information transfer [64]. This architecture contains a transceiver based on downlink or uplink information transfer, which either demodulates and decodes or encodes and modulates received data, and a component of harvested energy converts an input signal to DC power. Once the architecture is configured for downlink, the outputs of the antenna are logically combined to improve the power of received signals. Thereafter, the combiner output is splitted into two by the power splitter and is sent to both the transceiver and the energy harvester. Following this, the architecture configured for uplink by having attached two antennas separately to support the fullduplex operation for both information and power transfer in the transceiver and the energy harvester.

\section{O. Summary and Insights}

In this section, we reviewed existing literature related to the use of SWIPT in emerging communication technologies and provided future directions. The increasing attention to the energy efficiency of $5 \mathrm{G}$ has led research community to an extensive range of research opportunities in related to integrating SWIPT with modern communication technologies. First, we reviewed multi-carrier SWIPT systems with the latest literature. Most of the existing works have studied OFDM based SWIPT systems. Moreover, different SWIPT techniques and different multiple access schemes used in multi-carrier SWIPT systems are explained. Then we provided 


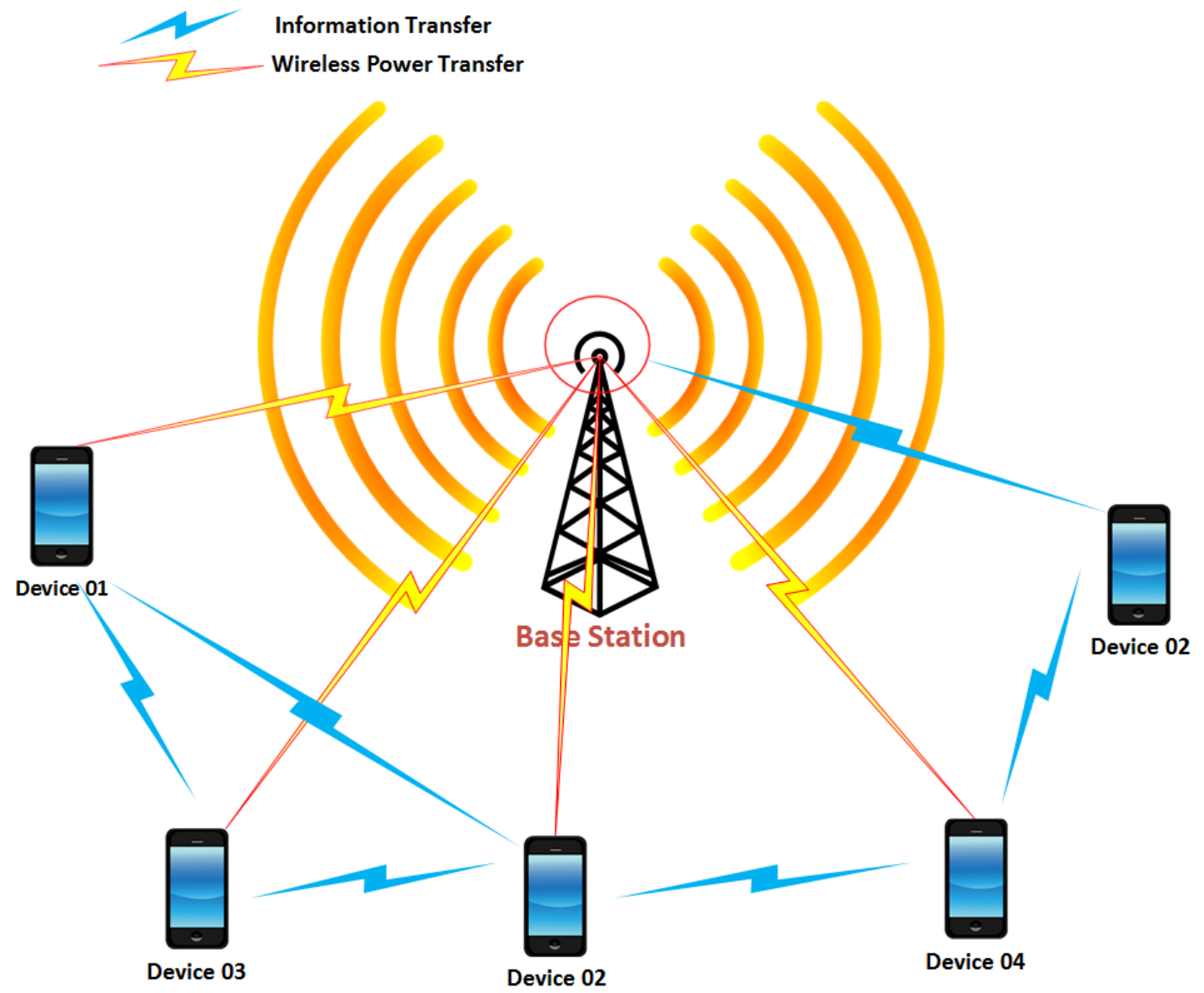

Fig. 14: SWIPT assisted D2D communication network where the users harvest energy from a base station and use harvested energy for D2D communication.

possible future directions in this context including the use of wideband receivers, investigated suitable techniques to utilize unused carriers for $\mathrm{EH}$ and explored the use of different sources for RF-EH in multi-carrier SWIPT systems. Next, we explained WPT/SWIPT enabled CRNs. Enabling SWIPT in CRNs can improve the spectral and energy efficiency of communication systems. A number of different CRN architectures with RF-EH have been proposed in the literature. Moreover, cooperative access schemes have been used to improve the efficiency of SWIPT transmissions in CRNs. Future research directions of SWIPT enabled CRNs should focus on the main four functions of CRNs, spectrum sensing, access, management and hand-off to support efficient and dynamic spectrum access in SWIPT enabled CRNs.

Next, we reviewed existing works of SWIPT enabled fullduplex communication. In the past, full-duplex communication was not encouraged to use in communication systems due to the effect of SI. However, with the finding of different uses of SI in communication systems, research community motivates to use full-duplex in modern communication systems. Principally, in SWIPT enabled full-duplex, SI can be converted into an extra source of energy. In recent research works related to SWIPT with FD communication have been used both TS and PS SWIPT receiver architectures. More importantly, some of the works applied TS and PS randomly to nodes in the communication systems to improve system efficiency. However, further research activities are required before SWIPT enabling full-duplex handshakes with modern communication systems. Furthermore, use of SI with related to interference exploitation need to be analysed and transceiver design needs to be changed accordingly in SWIPT enabled FD communication systems. An effective combination of full-duplex-CRNs with SWIPT was pointed out as a future direction. Then we reviewed Bistatic Scatter Radio for EH with proper explanation of literature available following by the future directions. The main research direction will be to find a reliable energy source for RF-EH in Bistatic Scatter Radio.

In the next subsection, we reviewed SWIPT with symbol level precoding. In this context, constructive interference can be used for ID and EH purposes in symbol level precoding. Few works have used SWIPT beamforming technique as a source for EH and a source of useful information signals. Investigating the feasibility of SWIPT systems with symbol level precoding in multi-cell systems can be one of the promising research directions. Next, we provided a comprehensive review on SWIPT enabled cooperative communication. SWIPT stands as a potential solution for the issue of using relay nodes its own energy to participate in relay operations. Moreover, we 


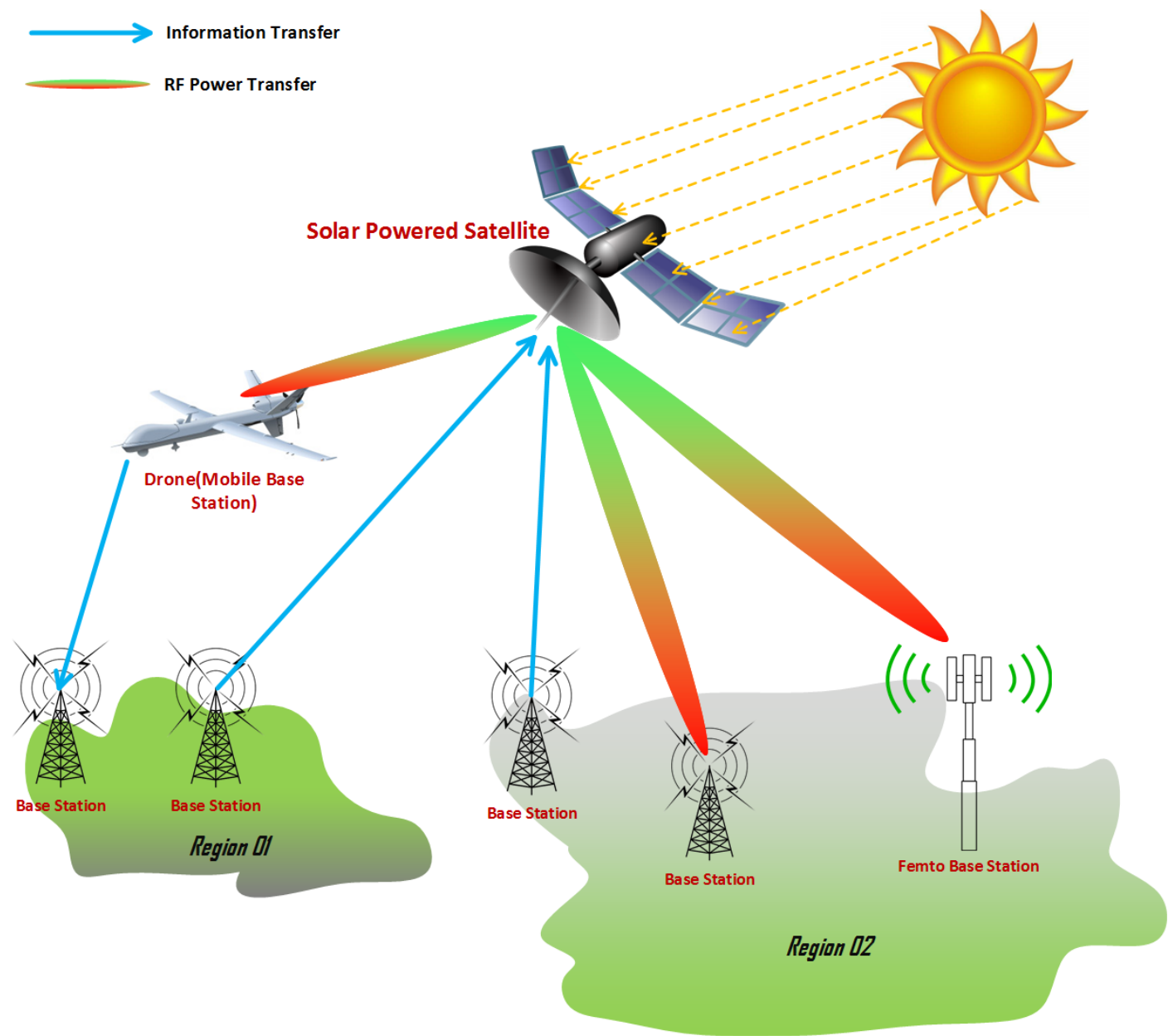

Fig. 15: SWIPT assisted solar powered satellite to initiate wireless power transmission and information transmission to ground and a mobile base station (drones or unmanned aerial vehicle) via microwave beams.

included aspects of cooperative relay algorithm design for resource allocation in SWIPT systems including, Harvest-thenCooperate, Harvest-use, self-energy recycling and interference aided harvesting. Most of the existing literature focused on using RF-EH and WPT to power up the relays. However, new SWIPT schemes need to be integrated with cooperative design protocols. Investigating cooperative communication models and the effect of channel codes can be a promising research direction. Next subsection included, SWIPT enabled cooperative NOMA. More attention came to NOMA with identifying as one of the major candidates in 5G. Although there are two different domains of NOMA, all existing literature on SWIPT assisted NOMA considered power-domain NOMA instead of the code-domain NOMA. Moreover, cooperative schemes have been used in SWIPT assisted NOMA to improve the reliability of remote users. Future research work in SWIPT assisted NOMA should further investigate SWIPT enabled NOMA using code-domain NOMA and with other communication technologies such as mmWave and MIMO. In addition, channel estimations and receiver design need to be addressed before integrating NOMA with SWIPT technology.

Next, we reviewed secure WPT/SWIPT transmission. In SWIPT systems, both information security and power transfer efficiency are equally important. Highly boosted signal emitted by the transmitter can lead to an expanded defencelessness against eavesdroppers. In the literature, separated receiver modes have been used as a solution, where one receiver allocated for confidential ID while the other one is assigned for EH. However, security issues in each and every emerging SWIPT enabled technologies need to be further investigated. SWIPT assisted mmWave communication was reviewed in the next subsection. mmWave communication has gained more attention after being selected as a key candidate for 5G. Very high frequency, narrow beam, large array gain and a dense network with mmWave base station make mmWave as a prominent candidate for WPT. Only a limited amount of works have considered mmWave for SWIPT and there is a huge gap in information processing and hardware development in SWIPT assisted mmWave communication. Next, we reviewed SWIPT enabled WSNs. SWIPT enabled WSNs TS and PS SWIPT architecture in EH wireless sensor nodes. As compared to sensing, data processing, communication is a costly functionality in SWIPT enabled WSNs. Thus, harvested power in nodes need to be used effectively to avoid catastrophic failure in the system. Furthermore, it is very important to identify potential energy sources for the EH process in WSNs. SWIPT enabled WSNs will lead the concept of IoT into a different level in the research community. Next, we reviewed 
SWIPT enabled MIMO systems that can notably improve the reliability and capacity of wireless networks. Initial research efforts focused on point to point MIMO links and now it has shifted to multi-user MIMO scenarios. SWIPT integration can be more beneficial since all receivers in MIMO are battery limited devices. SWIPT in massive MIMO enabled multiway relay networks used both TS and PS techniques and with the growth of the number of antennas in the systems improve the sum rate expression and harvested energy improve in a considerable amount. However, more research activities needed in long-distance power transfer to identify enabling solutions for path loss. Security aspects of SWIPT enabled MIMO need to be further investigated before applying into real-world communication. Finally, we have reviewed existing literature in SWIPT enabled D2D communication and BWSs with possible future directions.

\section{RECOMMENDATION FOR FUTURE DIRECTIONS}

In the previous sections, we have highlighted some of the technical issues in SWIPT enabled communication network systems. SWIPT presents a number of common technical challenges in different SWIPT enabled emerging communication technologies. In the following, we suggest some of the future research directions for common issues in SWIPT.

\section{A. Resource Scheduling in SWIPT}

As previously mentioned, scheduling is a critical factor in improving the system performance of SWIPT networks and to meet some QoS criteria under SWIPT. Due to practical limitations of the EH circuit, it is not feasible to have both $\mathrm{EH}$ and ID from the received signal. Therefore, optimized resource allocation algorithms are needed to increase the performance of SWIPT/EH system. In [177], the authors designed a resource allocation algorithm for SWIPT for a realistic nonlinear EH model and showed significant improvement in the system performance. Therefore, existing scheduling algorithms need to be optimized or new scheduling algorithms need to be created to achieve better performance in SWIPT enabled communication networks.

\section{B. Hardware Impairments}

Most research works related to SWIPT have not yet focused on hardware impairments in the SWIPT enabled communication networks. In wireless communication, there occur several hardware impairments such as in-phase/ quadraturephase (I/Q) imbalances, high-power amplifier non-linearity and oscillator phase noise due to the involved low quality hardware transceiver components, and they may significantly degrade the performance of various wireless systems. The effect of realistic relay transceiver structures on the outage probability and throughput of WPT has been analysed and confirmed in [178] based on two-way decode-and-forward (DF) cognitive network in the presence of transceiver impairment. Moreover, DF in EH systems with multiple antennas are also studied in [179] under the presence of transmitter hardware impairments. However, hardware impairments can have different effects on different SWIPT enabled communication systems. In this regard, it is an interesting research direction to exploit the effect of hardware impairments in different SWIPT enabled wireless communication systems. In addition, we strongly recommend that future research on SWIPT and SWIPT emerging technologies need to pay extra attention to hardware impairments since this can be one of the direct reasons for the system performance degradation of SWIPT enabled communication systems. Hopefully, the outcome of such research can be used to select appropriate quality hardware for future SWIPT enabled communication systems.

\section{Investigation of CSI Feedback}

Previous improvements in modulation, coding and scheduling have led to the wireless communication networks deployed in the present. Different channel adaptive techniques tend to be used in next-generation wireless networks. Thus, the transmitter requires some information regarding wireless channel conditions referred to as CSI at the transmitter. In SWIPT enabled next-generation communication networks, CSI could help to improve efficiency of both EH and ID operations. Since the value of the feedback varies with the communication scenario, investigating various types of feedback to improve the efficiency of SWIPT enabled communication systems could be a promising research direction. The selections of the best suitable feedback mechanism for SWIPT systems also can be a difficult choice to make since different communication systems have different characteristics and requirements. For instance, some capable terminals at the transmitter could afford CSI feedback, but not the simple IoT terminals. Therefore, how feedback mechanism affects the selection and effectiveness of the SWIPT strategy needs to be studied further in the nextgeneration communication systems.

\section{Channel Coding Techniques}

A proper channel coding technique is needed for SWIPT enabled communication systems since the receiving signal may contain corrupted information such as noise, interference, fading etc., and the available energy budget is extremely limited for self-powered nodes. Polar codes and LDPC codes can be potential channel coding techniques in SWIPT enabled 5G communications. A method for efficiently constructing polar codes has been presented and analysed in [180], and it has been applied in cooperative communications. Polar codes can be decoded by using successive cancellation (SC) or the belief propagation (BP) algorithms. However, unlike SC decoders, performance optimizations for BP decoders have not been very fully explored. An early stopping criteria for polar BP decoding to reduce energy dissipation and decoding latency has been proposed in [181] [182]. In [183], it has been shown that polar codes are suitable for DF and compress-and-forward relaying in relay channels with the orthogonal receivers.

A low-power decoder design approach for generic quasicyclic low-density parity-check (QC-LDPC) codes based on the layered min-sum decoding algorithm has been given in [184] and a low-power high-throughput LDPC decoder using 


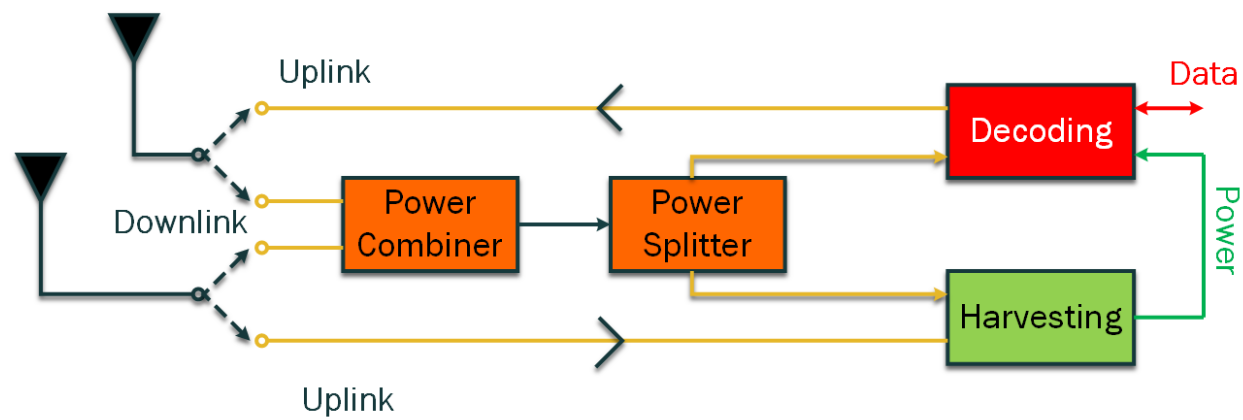

Fig. 16: Dual mode SWIPT supported mobile architecture: This architecture contains a transceiver based on downlink or uplink information transfer, which either demodulates and decodes or encodes and modulates received data, and a component of harvested energy converts an input signal to DC power.

non-refresh embedded DRAM has been designed in [185]. Turbo codes have been used in $3 \mathrm{G}$ cellular standard. These codes achieve lower bit error rates at the expense of highcomputational complexity as compared to LDPC and Polar codes. Therefore, for mobile communication devices, designing energy-efficient Turbo decoders is of great importance. In [186], the authors presented a MAP-based Turbo decoding algorithms with energy-quality trade-offs for various channel models.

However, an energy-efficient Polar and LDPC coding scheme for SWIPT enabled communication systems is required. The investigation of low power consumption and adaptive coding techniques suitable for SWIPT/WPT dependent wireless communication system is an encouraging research direction. The Turbo codes have the benefits of low interconnect complexity and inherent flexibility. In [187], the authors demonstrated that they facilitate high-throughput flexible channel coding gain at lower implementation complexities as compared to LDPC and polar codes. They further explained that the turbo codes offer an additional benefit of backward compatibility to $3 \mathrm{G}$ and $4 \mathrm{G}$ standards, which is favourable for the cellular communications industry in terms of cost savings. This again invites the research community on channel coding to investigate the best suitable coding technique for energyefficient deployment in $5 \mathrm{G}$ standards.

\section{E. Information Theoretic Framework}

Information theoretic framework assists to quantitatively analyse and model parameters in communication networks. A proper theoretical framework has not yet been developed for SWIPT technology. Since SWIPT has recently been introduced, a theoretical framework for trustworthiness evaluation could raise the possibility of merging SWIPT with existing communication technologies. In this regard, implementing theoretic frameworks for SWIPT systems can be an interesting and valuable research area.

\section{F. Internet of Things}

IoT is a novel concept which is based on connecting all types of electronic devices to the Internet. IoT basically connects people, processes, data and every possible things together in order to fulfill people's day-to-day needs. In the present, IoT is defined in the broader context comprising of different scenarios using different communication strategies such as WSNs, D2D and machine-to-machine communication. One of the main deployment challenges in IoT is to maintain reliable communication with the low cost and power limited IoT devices. The rapid growth of IoT applications has increased the energy consumption of small devices dramatically. Therefore, it is important to address the energy efficiency of small devices. In general, most of the IoT devices are battery operated and sometimes can be located in remote areas such as devices in a vessel in the middle of the deep ocean, military devices etc. [188]. Therefore, charging those batteries can sometimes be economically infeasible. Wireless relaying has been widely used in IoT wireless systems [189]. However, relaying operations require extra power from nodes in the relay, which sometimes can prevent battery powered devices from taking part in the relay. Therefore, RF powered relaying is a favourable solution, in which nodes in the relay can harvest energy either from source or externally from external RF transmissions. SWIPT integration to IoT devices can be a promising solution to improve efficiency of IoT. Nevertheless, the integration of SWIPT with IoT opens up new challenges. Only the energy used for transmission merely is take into account when designing scheduling. However, energy used for receiving cannot be ignored as compared to that of the transmission [190]. Thus, accurate energy modelling is required. Moreover, more works need to be carried out regarding relay selection in IoT, due to the fact that relay selection criteria for information transmission may be different from energy transfer. The distance between nodes in IoT networks will notably affect overall SWIPT system performance. Most of the existing energy harvesters work efficiently over short distances. Therefore, decisions have to be taken regarding the use of multi-hop with shorter distance in IoT networks.

\section{G. Machine type communications (MTC)}

MTC is a promising technology that enables communication between machine type devices such as devices/sensors, machines and vehicles, to network entities such as base stations, eNode base stations and access points with minimal human intervention.

A wide range of devices and applications can be involved in MTC. MTC applications may include health monitoring 
devices, transportation terminals, smart parking meters, etc. which must be effectively connected via communication links [191]. Nevertheless, some of these devices may have limited power capacity within the devices. Sometimes failure of a device in the linked network can have huge impact on day-today human activities. Thus, while improving communication aspects of the MTC, extra attention needs to be allocated for power requirement of the devices. SWIPT integration to MTC can be a revolutionary concept which helps to stabilise the power requirements of some devices in the network. Energy can be harvested using the same communication link while devices are in idle mode. Nevertheless, integration of SWIPT also can create some additional challenges. Some of the challenges are:

1) privacy concerns of the devices and the users

2) heterogeneity and mobility management of the devices

3) QoS requirement

MTC traffic is different from other communication networks. It has unique traffic patterns due to its special functionalities and requirements. These details need to be taken into consideration when designing ID and EH scheduling for the network. This can present another interesting research question in SWIPT enabled M2M communication. One of the main characteristics of the MTC, the capability of integrating many different types of devices, can also present a challenge in the integration of SWIPT. Available energy, computational power, power storage capacity and power requirement of the devices may be very different and these heterogeneity properties make the design of EH protocols a very challenging task. Security will be another important issue for the successful integration of SWIPT with MTC. Information and power transfer efficiency and information security are equally important in SWIPT enabled MTC. However, due to the different characteristics of devices in the network, security issues and possible solutions can vary. Therefore, considerations should be given to provide a satisfactory level of security and effective resource management schemes in SWIPT enabled MTC.

\section{H. Satellite Communication}

Satellite assisted SWIPT is an interesting application via a radio frequency beam. As many satellites are solar-powered, we could use solar EH to power up the satellite and then to use it to initiate wireless power transmission to ground stations via microwave beams. It could also participate in information transmission [192]. This is designed in a geostationary orbit, $36,000 \mathrm{~km}$ above the Earth's surface as shown in Fig. 15 This can be used for a SWIPT application, as the satellite can transmit information and power for low power consumed base stations and mobile base stations such as drones etc. This can also be used to energize UAVs while supporting the idea of ubiquitous energy. Future research should explore the development and implementation of such satellite assisted SWIPT based drones, base stations or similar applications and contribute to the idea of ubiquitous energy becoming a reality.

\section{CONCLUSION}

Exponential increase in wireless connectivity, increasing demand in wireless data usage due to ultra top notch mul- timedia contents and the rapid increase in the development of feature-rich smart devices are forcing us to look for the next major cellular evolution towards $5 \mathrm{G}$. The next-generation $5 \mathrm{G}$ systems are already providing hopes for the community due to a manifold increase in connectivity, data rate, QoS and QoE. The emerging applications like IoT, MTC and smart cities are expected to handshake with the next-generation $5 \mathrm{G}$ communication systems. The complexity of next-generation communication systems forced research community to look for better sustainable energy sources or to perform $\mathrm{EH}$ from external energy sources. Recently, the research community focused more on implementing self-sustainable communication systems while integrating novel concepts like RF-EH, WPT and SWIPT. In this paper, we have presented a comprehensive survey of RF-EH, WPT and SWIPT emerging technologies with recommendations and future directions.

First we pointed out the need for sustainable communication in 5G communications systems and reviewed existing relay articles related to SWIPT/WPT with the objective of highlighting the novelty of our survey paper. Subsequently, we provided a comprehensive description of RF-EH and the circuit design of EH module. Afterwards, we discussed WPT, including different technologies in WPT. Then we provided a detailed description of the SWIPT technology and its antenna architecture to provide foundational knowledge, which helps to understand the remaining contents of the paper. Next, we provided a detailed description of one of the uprising concepts, interference exploitation in SWIPT. This included the identification of new aspects of interference and the potential use of interference in wireless systems.

This paper then supplies comprehensive description of SWIPT emerging wireless communication technologies including multi-carrier SWIPT systems, WPT/SWIPT enabled CR, SWIPT enabled full-duplex, bistatic scatter radio, SWIPT with symbol level precoding, WPT/SWIPT on cooperative relaying, SWIPT enabled cooperative NOMA, secure SWIPT transmission, SWIPT assisted mmWave communications, SWIPT enabled WSNs, SWIPT assisted MIMO, SWIPT with two user MISO interference channel, SWIPT assisted D2D and SWIPT enabled BWSs. Moreover, we provided a comprehensive survey on up to date research works and future research directions of each specific emerging technology.

Finally, we pointed out major common technical challenges in different SWIPT enabled emerging communication technologies and future recommendations. The biggest challenge in the next-generation wireless communication is to integrate the spectrum of several enabling technologies to provide resource efficient, cost-effective and reliable communication. We believe that our survey article can be used as a guideline for major future research works in the next-generation SWIPT emerging technologies.

\section{APPENDIX}

TABLE VI provides the definitions of acronyms and notations used throughout this paper. 
TABLE VI: DEFINITIONS OF ACRONYMS AND NOTATIONS

\begin{tabular}{|c|c|c|c|}
\hline $\begin{array}{l}\text { Acronym } \\
\text { Notations }\end{array}$ & Definition & $\begin{array}{l}\text { Acronym } \\
\text { Notations }\end{array}$ & Definition \\
\hline $5 \mathrm{G}$ & Fifth generation & OFDMA & $\begin{array}{l}\text { Orthogonal Frequency Division Multiplexing } \\
\text { Access }\end{array}$ \\
\hline $\mathrm{AC}$ & Alternating Current & PS & Power Splitting \\
\hline BP & Belief Propagation & PTE & Power Transfer Efficiency \\
\hline BS & Base Station & QC & quasi-cyclic \\
\hline BWS & Broadband Wireless Systems & QoE & Quality of Experience \\
\hline $\mathrm{CCI}$ & Co-Channel Interference & QoS & Quality of Service \\
\hline CSI & Channel State Information & $\mathrm{RF}$ & Radio Frequency \\
\hline D2D & Device to Device Communication & RF-ENHs & Radio Frequency EH Networks \\
\hline DC & Direct Current & RFID & Radio Frequency identification \\
\hline $\mathrm{DF}$ & Decode and Forwarding & RIPT & Resonant Inductive Power Transfer \\
\hline DI & Data Information & $\mathrm{SC}$ & Successive Cancellation \\
\hline DL & Downlink & SI & Self Interference \\
\hline DRAM & Dynamic Random Access Memory & SIC & Successive Interference Cancellation \\
\hline $\mathrm{EH}$ & $\mathrm{EH}$ & SNIR & Signal to Interference plus Noise Ratio \\
\hline $\mathrm{EV}$ & Electronic Vehicle & SNR & Signal to Noise Ratio \\
\hline FD & Full-Duplex & SWIPT & Simultaneous Information and Power Transfer \\
\hline $\mathrm{I} / \mathrm{Q}$ & In-phase/Quadrature-phase & TDMA & Time Division Multiple Access \\
\hline IA & Interference Alignment & TS & Time Switching \\
\hline ID & Information Decoding & UAVs & Unmanned Aerial Vehicles \\
\hline IoT & Internet of Things & UE & User Equipment \\
\hline IPT & Inductive Power Transfer & UER & User Equipment Relay \\
\hline LDPC & Low-density Parity-check & UHF & Ultra High Frequency \\
\hline M2M & Machine to Machine Communication & UL & Uplink \\
\hline MAP & Maximum A Posteriori Decoding & WLAN & Wireless Local Area Network \\
\hline MIMO & Multiple Inputs Multiple Outputs & WPCN & Wireless Power Cellular Networks \\
\hline MISO & Multiple Input Single Output & WPT & Wireless Power Transfer \\
\hline mmWave & Millimeter Wave & WSNs & Wireless Sensor Networks \\
\hline $\mathrm{mmWC}$ & Millimeter Wave Communication & WWAN & Wireless Wide Area Network \\
\hline MPT & Microwave Power Transfer & $\eta$ & Energy Harvesting Efficiency Factor \\
\hline MTC & Machine-type communication devices & $P$ & Power \\
\hline MWRNs & Multiway Relay networks & $h$ & Channel Gain \\
\hline NOMA & Non Orthogonal Multiple Access & $W$ & Transmission Bandwidth \\
\hline $\mathrm{OC}$ & Opportunistic Communication & $\sigma^{2}$ & Noise Power \\
\hline OFDM & Orthogonal Frequency Division Multiplexing & $\theta$ & Power-Splitting Coefficient \\
\hline
\end{tabular}

\section{REFERENCES}

[1] J. G. Andrews, S. Buzzi, W. Choi, S. V. Hanly, A. Lozano, A. C. Soong, and J. C. Zhang, "What will 5g be?" IEEE Journal on selected areas in communications, vol. 32, no. 6, pp. 1065-1082, 2014.

[2] A. Osseiran, F. Boccardi, V. Braun, K. Kusume, P. Marsch, M. Maternia, O. Queseth, M. Schellmann, H. Schotten, H. Taoka et al., "Scenarios for $5 \mathrm{~g}$ mobile and wireless communications: the vision of the metis project," IEEE Communications Magazine, vol. 52, no. 5, pp. 26-35, 2014.

[3] M. Agiwal, A. Roy, and N. Saxena, "Next generation 5g wireless networks: A comprehensive survey," IEEE Communications Surveys \& Tutorials, vol. 18, no. 3, pp. 1617-1655, 2016.

[4] Q. Wu, G. Y. Li, W. Chen, D. W. K. Ng, and R. Schober, "An overview of sustainable green $5 \mathrm{~g}$ networks," IEEE Wireless Communications, vol. 24, no. 4, pp. 72-80, 2017.

[5] D. Niyato, E. Hossain, M. M. Rashid, and V. K. Bhargava, "Wireless sensor networks with energy harvesting technologies: A game-theoretic approach to optimal energy management," IEEE Wireless Communications, vol. 14, no. 4, 2007.

[6] L. Hou and S. Tan, "A preliminary study of thermal energy harvesting for industrial wireless sensor networks," in Sensing Technology (ICST), 2016 10th International Conference on. IEEE, 2016, pp. 1-5.
[7] I. Krikidis, S. Timotheou, S. Nikolaou, G. Zheng, D. W. K. Ng, and R. Schober, "Simultaneous wireless information and power transfer in modern communication systems," IEEE Communications Magazine, vol. 52, no. 11, pp. 104-110, 2014.

[8] L. R. Varshney, "Transporting information and energy simultaneously," in Information Theory, 2008. ISIT 2008. IEEE International Symposium on. IEEE, 2008, pp. 1612-1616.

[9] I.-J. Yoon, "Wireless power transfer in the radiating near-field region," in Radio Science Meeting (Joint with AP-S Symposium), 2015 USNCURSI. IEEE, 2015, pp. 344-344.

[10] K. Huang and X. Zhou, "Cutting the last wires for mobile communications by microwave power transfer," IEEE Communications Magazine, vol. 53, no. 6, pp. 86-93, 2015.

[11] X. Lu, P. Wang, D. Niyato, D. I. Kim, and Z. Han, "Wireless networks with rf energy harvesting: A contemporary survey," IEEE Communications Surveys \& Tutorials, vol. 17, no. 2, pp. 757-789, 2015.

[12] H. Yu, L. Shi, Y. Qian, F. Shu, J. Li, Y. Zhao, and D. N. K. Jayakody, "A cooperative modulation recognition: New paradigm for power line networks in smart grid," Physical Communication, 2017.

[13] Z. Ding, C. Zhong, D. W. K. Ng, M. Peng, H. A. Suraweera, R. Schober, and H. V. Poor, "Application of smart antenna technologies in simultaneous wireless information and power transfer," IEEE 
Communications Magazine, vol. 53, no. 4, pp. 86-93, 2015.

[14] S. Ulukus, A. Yener, E. Erkip, O. Simeone, M. Zorzi, P. Grover, and K. Huang, "Energy harvesting wireless communications: A review of recent advances," IEEE Journal on Selected Areas in Communications, vol. 33, no. 3, pp. 360-381, 2015.

[15] X. Chen, D. W. K. Ng, and H.-H. Chen, "Secrecy wireless information and power transfer: Challenges and opportunities," IEEE Wireless Communications, vol. 23, no. 2, pp. 54-61, 2016.

[16] M. Amjad, F. Akhtar, M. H. Rehmani, M. Reisslein, and T. Umer, "Full-duplex communication in cognitive radio networks: A survey," IEEE Communications Surveys \& Tutorials, 2017.

[17] X. Lu, P. Wang, D. Niyato, D. I. Kim, and Z. Han, "Wireless charging technologies: Fundamentals, standards, and network applications," IEEE Communications Surveys \& Tutorials, vol. 18, no. 2, pp. 14131452, 2016.

[18] N. Zhao, S. Zhang, R. Yu, Y. Chen, A. Nallanathan, and V. Leung, "Exploiting interference for energy harvesting: a survey, research issues and challenges," IEEE Access, 2017.

[19] M.-L. Ku, W. Li, Y. Chen, and K. R. Liu, "Advances in energy harvesting communications: Past, present, and future challenges," IEEE Communications Surveys \& Tutorials, vol. 18, no. 2, pp. 1384-1412, 2016.

[20] O. Ozel, K. Tutuncuoglu, S. Ulukus, and A. Yener, "Fundamental limits of energy harvesting communications," IEEE Communications Magazine, vol. 53, no. 4, pp. 126-132, 2015.

[21] H. J. Visser and R. J. Vullers, "Rf energy harvesting and transport for wireless sensor network applications: Principles and requirements," Proceedings of the IEEE, vol. 101, no. 6, pp. 1410-1423, 2013.

[22] F. Akhtar and M. H. Rehmani, "Energy harvesting for self-sustainable wireless body area networks," IT Professional, vol. 19, no. 2, pp. 32 40, 2017.

[23] F. Akhtar and M. Rehmani, "Energy replenishment using renewable and traditional energy resources for sustainable wireless sensor networks: A review," Renewable and Sustainable Energy Reviews, vol. 45, pp. 769-784, 2015.

[24] H. Nishimoto, Y. Kawahara, and T. Asami, "Prototype implementation of ambient rf energy harvesting wireless sensor networks," in Sensors, 2010 IEEE. IEEE, 2010, pp. 1282-1287.

[25] N. Alliance, "5g white paper," Next generation mobile networks, white paper, 2015.

[26] X. Lu, P. Wang, D. Niyato, and E. Hossain, "Dynamic spectrum access in cognitive radio networks with rf energy harvesting," IEEE Wireless Communications, vol. 21, no. 3, pp. 102-110, 2014.

[27] J. Akkermans, M. Van Beurden, G. Doodeman, and H. Visser, "Analytical models for low-power rectenna design," IEEE Antennas and Wireless Propagation Letters, vol. 4, no. 1, pp. 187-190, 2005.

[28] X. Zhang, H. Jiang, L. Zhang, C. Zhang, Z. Wang, and X. Chen, "An energy-efficient asic for wireless body sensor networks in medical applications," IEEE transactions on biomedical circuits and systems, vol. 4, no. 1, pp. 11-18, 2010.

[29] R. C. Johnson, H. A. Ecker, and J. S. Hollis, "Determination of farfield antenna patterns from near-field measurements," Proceedings of the IEEE, vol. 61, no. 12, pp. 1668-1694, 1973.

[30] S. Shrestha, S.-K. Noh, and D.-Y. Choi, "Comparative study of antenna designs for rf energy harvesting," International Journal of Antennas and Propagation, vol. 2013, 2013.

[31] R. W. Habash, J. M. Elwood, D. Krewski, W. G. Lotz, J. P. McNamee, and F. S. Prato, "Recent advances in research on radio frequency fields and health: 2004-2007," Journal of Toxicology and Environmental Health, Part B, vol. 12, no. 4, pp. 250-288, 2009.

[32] C. Branch, "Limits of human exposure to radio frequency electromagnetic energy in the frequency range from $3 \mathrm{khz}$ to $300 \mathrm{ghz}$," safe code, vol. 6, 2009.

[33] R. E. Hamam, A. Karalis, J. Joannopoulos, and M. Soljačić, "Efficient weakly-radiative wireless energy transfer: An eit-like approach," Annals of Physics, vol. 324, no. 8, pp. 1783-1795, 2009.

[34] S. Ho, J. Wang, W. Fu, and M. Sun, "A comparative study between novel witricity and traditional inductive magnetic coupling in wireless charging," IEEE Transactions on Magnetics, vol. 47, no. 5, pp. 1522 1525, 2011.

[35] J. G. Hayes, M. G. Egan, J. M. Murphy, S. E. Schulz, and J. T. Hall, "Wide-load-range resonant converter supplying the sae j-1773 electric vehicle inductive charging interface," IEEE Transactions on Industry Applications, vol. 35, no. 4, pp. 884-895, 1999.

[36] T. Nagashima, X. Wei, E. Bou, E. Alarcón, M. K. Kazimierczuk, and H. Sekiya, "Analysis and design of loosely inductive coupled wireless power transfer system based on class- $e^{2} \mathrm{dc}$-dc converter for efficiency enhancement," IEEE Transactions on Circuits and Systems I: Regular Papers, vol. 62, no. 11, pp. 2781-2791, 2015.

[37] B. Lee, M. Kiani, and M. Ghovanloo, "A triple-loop inductive power transmission system for biomedical applications," IEEE transactions on biomedical circuits and systems, vol. 10, no. 1, pp. 138-148, 2016.

[38] A. Kurs, A. Karalis, R. Moffatt, J. D. Joannopoulos, P. Fisher, and M. Soljačić, "Wireless power transfer via strongly coupled magnetic resonances," science, vol. 317, no. 5834, pp. 83-86, 2007.

[39] G. A. Covic, J. T. Boys, M. L. Kissin, and H. G. Lu, "A three-phase inductive power transfer system for roadway-powered vehicles," IEEE Transactions on Industrial Electronics, vol. 54, no. 6, pp. 3370-3378, 2007.

[40] E. Bou-Balust, A. P. Hu, and E. Alarcon, "Scalability analysis of simo non-radiative resonant wireless power transfer systems based on circuit models," IEEE Transactions on Circuits and Systems I: Regular Papers, vol. 62, no. 10, pp. 2574-2583, 2015.

[41] M. Chabalko, J. Besnoff, M. Laifenfeld, and D. S. Ricketts, "Resonantly coupled wireless power transfer for non-stationary loads with application in automotive environments," IEEE Transactions on Industrial Electronics, vol. 64, no. 1, pp. 91-103, 2017.

[42] J. Zhang and C. Cheng, "Comparative studies between kvl and bpft in magnetically-coupled resonant wireless power transfer," IET Power Electronics, vol. 9, no. 10, pp. 2121-2129, 2016.

[43] A. P. Sample, D. T. Meyer, and J. R. Smith, "Analysis, experimental results, and range adaptation of magnetically coupled resonators for wireless power transfer," IEEE Transactions on Industrial Electronics, vol. 58, no. 2, pp. 544-554, 2011.

[44] J. Dai and D. C. Ludois, "A survey of wireless power transfer and a critical comparison of inductive and capacitive coupling for small gap applications," IEEE Transactions on Power Electronics, vol. 30, no. 11, pp. 6017-6029, 2015

[45] J. Kim, D.-H. Kim, and Y.-J. Park, "Analysis of capacitive impedance matching networks for simultaneous wireless power transfer to multiple devices," IEEE Transactions on Industrial Electronics, vol. 62, no. 5, pp. 2807-2813, 2015.

[46] F. Lu, H. Zhang, H. Hofmann, and C. C. Mi, "An inductive and capacitive combined wireless power transfer system with lc-compensated topology," IEEE Transactions on Power Electronics, vol. 31, no. 12, pp. 8471-8482, 2016.

[47] J. Dai and D. C. Ludois, "Capacitive power transfer through a conformal bumper for electric vehicle charging," IEEE Journal of Emerging and Selected Topics in Power Electronics, vol. 4, no. 3, pp. 1015-1025, 2016.

[48] A. Massa, G. Oliveri, F. Viani, and P. Rocca, "Array designs for longdistance wireless power transmission: State-of-the-art and innovative solutions," Proceedings of the IEEE, vol. 101, no. 6, pp. 1464-1481, 2013.

[49] G. A. Landis, "Applications for space power by laser transmission," in PROCEEDINGS-SPIE THE INTERNATIONAL SOCIETY FOR OPTICAL ENGINEERING. SPIE INTERNATIONAL SOCIETY FOR OPTICAL, 1994, pp. 252-252.

[50] G. Landis, "Re-evaluating satellite solar power systems for earth," in Photovoltaic Energy Conversion, Conference Record of the 2006 IEEE 4th World Conference on, vol. 2. IEEE, 2006, pp. 1939-1942.

[51] M. Xia and S. Aissa, "On the efficiency of far-field wireless power transfer," IEEE transactions on signal processing, vol. 63, no. 11, pp. 2835-2847, 2015

[52] J. Park, Y. Tak, Y. Kim, Y. Kim, and S. Nam, "Investigation of adaptive matching methods for near-field wireless power transfer," IEEE Transactions on Antennas and Propagation, vol. 59, no. 5, pp. 1769-1773, 2011.

[53] M. Bhutada, V. Singh, and C. Warty, "Transmission ofwireless power in two-coil and four-coil systems using coupled mode theory," in Aerospace Conference, 2015 IEEE. IEEE, 2015, pp. 1-8.

[54] J. Lee and S. Nam, "Fundamental aspects of near-field coupling small antennas for wireless power transfer," IEEE Transactions on Antennas and Propagation, vol. 58, no. 11, pp. 3442-3449, 2010.

[55] S. Y. R. Hui, W. Zhong, and C. K. Lee, "A critical review of recent progress in mid-range wireless power transfer," IEEE Transactions on Power Electronics, vol. 29, no. 9, pp. 4500-4511, 2014.

[56] W. C. Brown, "The history of power transmission by radio waves," IEEE Transactions on microwave theory and techniques, vol. 32, no. 9 , pp. 1230-1242, 1984.

[57] J. O. McSpadden and J. C. Mankins, "Space solar power programs and microwave wireless power transmission technology," IEEE microwave magazine, vol. 3, no. 4, pp. 46-57, 2002. 
[58] R. Zhang and C. K. Ho, "Mimo broadcasting for simultaneous wireless information and power transfer," IEEE Transactions on Wireless Communications, vol. 12, no. 5, pp. 1989-2001, 2013.

[59] D. W. K. Ng, E. S. Lo, and R. Schober, "Robust beamforming for secure communication in systems with wireless information and power transfer," IEEE Transactions on Wireless Communications, vol. 13, no. 8, pp. 4599-4615, 2014.

[60] D. Ng, E. S. Lo, and R. Schober, "Wireless information and power transfer: Energy efficiency optimization in ofdma systems," IEEE Transactions on Wireless Communications, vol. 12, no. 12, pp. 63526370, 2013.

[61] X. Zhou, "Training-based swipt: Optimal power splitting at the receiver," IEEE Transactions on Vehicular Technology, vol. 64, no. 9, pp. 4377-4382, 2015.

[62] X. Zhou, R. Zhang, and C. K. Ho, "Wireless information and power transfer: Architecture design and rate-energy tradeoff," IEEE Transactions on Communications, vol. 61, no. 11, pp. 4754-4767, 2013.

[63] I. Krikidis, S. Sasaki, S. Timotheou, and Z. Ding, "A low complexity antenna switching for joint wireless information and energy transfer in mimo relay channels," IEEE Transactions on Communications, vol. 62 , no. 5, pp. $1577-1587,2014$

[64] K. Huang and E. Larsson, "Simultaneous information and power transfer for broadband wireless systems," IEEE Transactions on Signal Processing, vol. 61, no. 23, pp. 5972-5986, 2013.

[65] C. Shen, W.-C. Li, and T.-H. Chang, "Simultaneous information and energy transfer: A two-user miso interference channel case," in Global Communications Conference (GLOBECOM), 2012 IEEE. IEEE, 2012, pp. 3862-3867.

[66] X. Zhou, R. Zhang, and C. K. Ho, "Wireless information and power transfer in multiuser ofdm systems," IEEE Transactions on Wireless Communications, vol. 13, no. 4, pp. 2282-2294, 2014.

[67] Z. Hu, C. Yuan, F. Zhu, and F. Gao, "Weighted sum transmit power minimization for full-duplex system with swipt and self-energy recycling," IEEE Access, vol. 4, pp. 4874-4881, 2016.

[68] M. Gao, H. H. Chen, Y. Li, M. Shirvanimoghaddam, and J. Shi, "Fullduplex wireless-powered communication with antenna pair selection," in Wireless Communications and Networking Conference (WCNC), 2015 IEEE. IEEE, 2015, pp. 693-698.

[69] V. C. Gungor and G. P. Hancke, "Industrial wireless sensor networks: Challenges, design principles, and technical approaches," IEEE Transactions on industrial electronics, vol. 56, no. 10, pp. 4258-4265, 2009.

[70] L. Lu, G. Y. Li, A. L. Swindlehurst, A. Ashikhmin, and R. Zhang, "An overview of massive mimo: Benefits and challenges," IEEE journal of selected topics in signal processing, vol. 8, no. 5, pp. 742-758, 2014.

[71] C. Masouros, T. Ratnarajah, M. Sellathurai, C. B. Papadias, and A. K. Shukla, "Known interference in the cellular downlink: A performance limiting factor or a source of green signal power?" IEEE Communications Magazine, vol. 51, no. 10, pp. 162-171, 2013.

[72] G. Zheng, I. Krikidis, C. Masouros, S. Timotheou, D.-A. Toumpakaris, and Z. Ding, "Rethinking the role of interference in wireless networks," IEEE Communications Magazine, vol. 52, no. 11, pp. 152-158, 2014.

[73] Y. Gu and S. Aissa, "Interference aided energy harvesting in decodeand-forward relaying systems," in Communications (ICC), 2014 IEEE International Conference on. IEEE, 2014, pp. 5378-5382.

[74] C. Masouros, "Harvesting signal power from constructive interference in multiuser downlinks," in Wireless Information and Power Transfer: A New Paradigm for Green Communications. Springer, 2018, pp. 87-122.

[75] C. Psomas and I. Krikidis, "Successive interference cancellation in bipolar ad hoc networks with swipt," IEEE Wireless Communications Letters, vol. 5, no. 4, pp. 364-367, 2016.

[76] J. Park and B. Clerckx, "Joint wireless information and energy transfer in a $k$-user mimo interference channel," IEEE Transactions on Wireless Communications, vol. 13, no. 10, pp. 5781-5796, 2014.

[77] I. Krikidis, "Simultaneous information and energy transfer in largescale networks with/without relaying," IEEE Transactions on Communications, vol. 62, no. 3, pp. 900-912, 2014.

[78] T. A. Khan, A. Alkhateeb, and R. W. Heath, "Millimeter wave energy harvesting," IEEE Transactions on Wireless Communications, vol. 15, no. 9, pp. 6048-6062, 2016.

[79] M. Alodeh, S. Chatzinotas, and B. Ottersten, "Constructive multiuser interference in symbol level precoding for the miso downlink channel," IEEE Transactions on Signal processing, vol. 63, no. 9, pp. 2239-2252, 2015.

[80] M. A, S. Chatzinotas, and B. Ottersten, "Energy-efficient symbol-level precoding in multiuser miso based on relaxed detection region," IEEE transactions on Wireless Communications, vol. 15, no. 5, pp. 37553767, 2016.

[81] C. Masouros and G. Zheng, "Exploiting known interference as green signal power for downlink beamforming optimization," IEEE Transactions on Signal processing, vol. 63, no. 14, pp. 3628-3640, 2015.

[82] D. B. da Costa, H. Ding, and J. Ge, "Interference-limited relaying transmissions in dual-hop cooperative networks over nakagami-m fading," IEEE Communications Letters, vol. 15, no. 5, pp. 503-505, 2011.

[83] N. Zhao, F. R. Yu, and V. C. Leung, "Opportunistic communications in interference alignment networks with wireless power transfer," IEEE Wireless Communications, vol. 22, no. 1, pp. 88-95, 2015.

[84] S. Timotheou, G. Zheng, C. Masouros, and I. Krikidis, "Exploiting constructive interference for simultaneous wireless information and power transfer in multiuser downlink systems," IEEE Journal on Selected Areas in Communications, vol. 34, no. 5, pp. 1772-1784, 2016.

[85] A. Shojaeifard, K.-K. Wong, M. Di Renzo, G. Zheng, K. A. Hamdi, and J. Tang, "Self-interference in full-duplex multi-user mimo channels," IEEE Communications Letters, vol. 21, no. 4, pp. 841-844, 2017.

[86] P. Grover and A. Sahai, "Shannon meets tesla: Wireless information and power transfer," in Information Theory Proceedings (ISIT), 2010 IEEE International Symposium on. IEEE, 2010, pp. 2363-2367.

[87] A. Naeem, M. H. Rehmani, Y. Saleem, I. Rashid, and N. Crespi, "Network coding in cognitive radio networks: A comprehensive survey," IEEE Communications Surveys \& Tutorials, 2017.

[88] Z. Hu, N. Wei, and Z. Zhang, "Optimal resource allocation for harvested energy maximization in wideband cognitive radio network with swipt," IEEE Access, 2017.

[89] A. El Shafie, N. Al-Dhahir, and R. Hamila, "Cooperative access schemes for efficient swipt transmissions in cognitive radio networks," in Globecom Workshops (GC Wkshps), 2015 IEEE. IEEE, 2015, pp. $1-6$.

[90] S. K. Sharma, T. E. Bogale, S. Chatzinotas, B. Ottersten, L. B. Le, and $\mathrm{X}$. Wang, "Cognitive radio techniques under practical imperfections: A survey," IEEE Communications Surveys Tutorials, vol. 17, no. 4, pp. 1858-1884, Fourthquarter 2015.

[91] J. I. Choi, M. Jain, K. Srinivasan, P. Levis, and S. Katti, "Achieving single channel, full duplex wireless communication," in Proceedings of the sixteenth annual international conference on Mobile computing and networking. ACM, 2010, pp. 1-12.

[92] D. Jayakody, J. Thompson, S. Chatzinotas, and S.Durani, Wireless Informationa and Power Transfer: A New Green Communications Paradigm. Springer-Verlag, 2017.

[93] M. Duarte, C. Dick, and A. Sabharwal, "Experiment-driven characterization of full-duplex wireless systems," IEEE Transactions on Wireless Communications, vol. 11, no. 12, pp. 4296-4307, 2012.

[94] E. Aryafar, M. A. Khojastepour, K. Sundaresan, S. Rangarajan, and M. Chiang, "Midu: Enabling mimo full duplex," in Proceedings of the 18th annual international conference on Mobile computing and networking. ACM, 2012, pp. 257-268.

[95] E. Ahmed and A. M. Eltawil, "All-digital self-interference cancellation technique for full-duplex systems," IEEE Transactions on Wireless Communications, vol. 14, no. 7, pp. 3519-3532, 2015.

[96] A. Sabharwal, P. Schniter, D. Guo, D. W. Bliss, S. Rangarajan, and R. Wichman, "In-band full-duplex wireless: Challenges and opportunities," IEEE Journal on Selected Areas in Communications, vol. 32 , no. 9, pp. 1637-1652, 2014.

[97] A. A. Okandeji, M. R. Khandaker, and K.-K. Wong, "Wireless information and power transfer in full-duplex communication systems," in Communications (ICC), 2016 IEEE International Conference on. IEEE, 2016, pp. 1-6.

[98] Y. Zeng and R. Zhang, "Full-duplex wireless-powered relay with selfenergy recycling," IEEE Wireless Communications Letters, vol. 4, no. 2, pp. 201-204, 2015.

[99] M. Maso, C.-F. Liu, C.-H. Lee, T. Q. Quek, and L. S. Cardoso, "Energy-recycling full-duplex radios for next-generation networks," IEEE Journal on Selected Areas in Communications, vol. 33, no. 12, pp. 2948-2962, 2015.

[100] C. Zhong, H. A. Suraweera, G. Zheng, I. Krikidis, and Z. Zhang, "Wireless information and power transfer with full duplex relaying," IEEE Transactions on Communications, vol. 62, no. 10, pp. 3447-3461, 2014

[101] H. Ju and R. Zhang, "Optimal resource allocation in full-duplex wireless-powered communication network," IEEE Transactions on Communications, vol. 62, no. 10, pp. 3528-3540, 2014. 
[102] X. Kang, C. K. Ho, and S. Sun, "Full-duplex wireless-powered communication network with energy causality," IEEE Transactions on Wireless Communications, vol. 14, no. 10, pp. 5539-5551, 2015.

[103] S. Leng, D. W. K. Ng, N. Zlatanov, and R. Schober, "Multi-objective resource allocation in full-duplex swipt systems," in Communications (ICC), 2016 IEEE International Conference on. IEEE, 2016, pp. 1-7.

[104] V.-D. Nguyen, H. V. Nguyen, G.-M. Kang, H. M. Kim, and O.-S Shin, "Sum rate maximization for full duplex wireless-powered communication networks," in Signal Processing Conference (EUSIPCO), 2016 24th European. IEEE, 2016, pp. 798-802.

[105] C. Zhong, H. A. Suraweera, G. Zheng, I. Krikidis, and Z. Zhang, "Wireless information and power transfer with full duplex relaying," IEEE Transactions on Communications, vol. 62, no. 10, pp. 3447-3461, 2014

[106] H. Stockman, "Communication by means of reflected power," Proceedings of the IRE, vol. 36, no. 10, pp. 1196-1204, 1948.

[107] G. Vannucci, A. Bletsas, and D. Leigh, "A software-defined radio system for backscatter sensor networks," IEEE Transactions on Wireless Communications, vol. 7, no. 6, 2008.

[108] N. Fasarakis-Hilliard, P. N. Alevizos, and A. Bletsas, "Coherent detection and channel coding for bistatic scatter radio sensor networking," IEEE Transactions on Communications, vol. 63, no. 5, pp. 1798-1810, 2015

[109] D. Kwon, H. S. Kang, and D. K. Kim, "Robust interference exploitation-based precoding scheme with quantized csit," IEEE Communications Letters, vol. 20, no. 4, pp. 780-783, 2016.

[110] S. Timotheou, G. Zheng, C. Masouros, and I. Krikidis, "Symbollevel precoding in miso broadcast channels for swipt systems," in Telecommunications (ICT), 2016 23rd International Conference on. IEEE, 2016, pp. 1-5.

[111] M. Alodeh, S. Chatzinotas, and B. Ottersten, "Constructive interference through symbol level precoding for multi-level modulation," in Global Communications Conference (GLOBECOM), 2015 IEEE. IEEE, 2015, pp. 1-6.

[112] Q. Shi, L. Liu, W. Xu, and R. Zhang, "Joint transmit beamforming and receive power splitting for miso swipt systems," IEEE Transactions on Wireless Communications, vol. 13, no. 6, pp. 3269-3280, 2014.

[113] M. Peng, Y. Liu, D. Wei, W. Wang, and H.-H. Chen, "Hierarchical cooperative relay based heterogeneous networks," IEEE Wireless Communications, vol. 18, no. 3, 2011.

[114] B. Zhou, H. Hu, S.-Q. Huang, and H.-H. Chen, "Intracluster deviceto-device relay algorithm with optimal resource utilization," IEEE transactions on vehicular technology, vol. 62 , no. 5, pp. 2315-2326, 2013.

[115] J. N. Laneman and G. W. Wornell, "Energy-efficient antenna sharing and relaying for wireless networks," in Wireless Communications and Networking Confernce, 2000. WCNC. 2000 IEEE, vol. 1. IEEE, 2000, pp. 7-12.

[116] J. N. Laneman, D. N. Tse, and G. W. Wornell, "Cooperative diversity in wireless networks: Efficient protocols and outage behavior," IEEE Transactions on Information theory, vol. 50, no. 12, pp. 3062-3080, 2004

[117] H. Chen, Y. Li, J. L. Rebelatto, B. F. Uchôa Filho, and B. Vucetic, "Harvest-then-cooperate: Wireless-powered cooperative communications." IEEE Trans. Signal Processing, vol. 63, no. 7, pp. 1700-1711, 2015

[118] A. Rajaram, D. N. K. Jayakody, and V. Skachek, "Store-then-cooperate: Energy harvesting scheme in cooperative relay networks," in Wireless Communication Systems (ISWCS), 2016 International Symposium on. IEEE, 2016, pp. 445-450.

[119] W. Huang, H. Chen, Y. Li, and B. Vucetic, "On the performance of multi-antenna wireless-powered communications with energy beamforming," IEEE Transactions on Vehicular Technology, vol. 65, no. 3 , pp. 1801-1808, 2016.

[120] I. Krikidis, G. Zheng, and B. Ottersten, "Harvest-use cooperative networks with half/full-duplex relaying," in Wireless Communications and Networking Conference (WCNC), 2013 IEEE. IEEE, 2013, pp. 4256-4260.

[121] A. A. Nasir, X. Zhou, S. Durrani, and R. A. Kennedy, "Relaying protocols for wireless energy harvesting and information processing," IEEE Transactions on Wireless Communications, vol. 12, no. 7, pp 3622-3636, 2013.

[122] Z. Ding, I. Krikidis, B. Sharif, and H. V. Poor, "Wireless information and power transfer in cooperative networks with spatially random relays," IEEE Transactions on Wireless Communications, vol. 13, no. 8, pp. 4440-4453, 2014.
[123] S. R. Islam, N. Avazov, O. A. Dobre, and K.-S. Kwak, "Power-domain non-orthogonal multiple access (noma) in $5 \mathrm{~g}$ systems: potentials and challenges," IEEE Communications Surveys \& Tutorials, 2017.

[124] Z. Ding, X. Lei, G. K. Karagiannidis, R. Schober, J. Yuan, and V. Bhargava, "A survey on non-orthogonal multiple access for $5 \mathrm{~g}$ networks: Research challenges and future trends," IEEE Journal on Selected Areas in Communications, 2017.

[125] Z. Ding, M. Peng, and H. V. Poor, "Cooperative non-orthogonal multiple access in 5g systems," IEEE Communications Letters, vol. 19, no. 8, pp. 1462-1465, 2015.

[126] Y. Liu, Z. Ding, M. Elkashlan, and H. V. Poor, "Cooperative nonorthogonal multiple access with simultaneous wireless information and power transfer," IEEE Journal on Selected Areas in Communications, vol. 34, no. 4, pp. 938-953, 2016.

[127] Z. Chen, Z. Ding, X. Dai, and G. K. Karagiannidis, "On the application of quasi-degradation to miso-noma downlink," IEEE Transactions on Signal Processing, vol. 64, no. 23, pp. 6174-6189, 2016.

[128] Z. Chen, Z. Ding, P. Xu, and X. Dai, "Optimal precoding for a qos optimization problem in two-user miso-noma downlink," IEEE Communications Letters, vol. 20, no. 6, pp. 1263-1266, 2016.

[129] N. T. Do, D. B. da Costa, T. Q. Duong, and B. An, "Transmit antenna selection schemes for miso-noma cooperative downlink transmissions with hybrid swipt protocol," in Communications (ICC), 2017 IEEE International Conference on. IEEE, 2017, pp. 1-6.

[130] H. Xing, K.-K. Wong, A. Nallanathan, and R. Zhang, "Wireless powered cooperative jamming for secrecy multi-af relaying networks," IEEE Transactions on Wireless Communications, vol. 15, no. 12, pp. 7971-7984, 2016.

[131] L. Dong, Z. Han, A. P. Petropulu, and H. V. Poor, "Improving wireless physical layer security via cooperating relays," IEEE transactions on signal processing, vol. 58, no. 3, pp. 1875-1888, 2010.

[132] J. Li, A. P. Petropulu, and S. Weber, "On cooperative relaying schemes for wireless physical layer security," IEEE Transactions on Signal Processing, vol. 59, no. 10, pp. 4985-4997, 2011.

[133] D. W. K. Ng, E. S. Lo, and R. Schober, "Robust beamforming for secure communication in systems with wireless information and power transfer," IEEE Transactions on Wireless Communications, vol. 13, no. 8, pp. 4599-4615, 2014

[134] M. Zhang and Y. Liu, "Energy harvesting for physical-layer security in ofdma networks," IEEE Transactions on Information Forensics and Security, vol. 11, no. 1, pp. 154-162, 2016

[135] M. Zhang, Y. Liu, and R. Zhang, "Artificial noise aided secrecy information and power transfer in ofdma systems," IEEE Transactions on Wireless Communications, vol. 15, no. 4, pp. 3085-3096, 2016.

[136] G. Pan, C. Tang, T. Li, and Y. Chen, "Secrecy performance analysis for simo simultaneous wireless information and power transfer systems," IEEE Transactions on Communications, vol. 63, no. 9, pp. 3423-3433, 2015.

[137] G. Pan, H. Lei, Y. Deng, L. Fan, J. Yang, Y. Chen, and Z. Ding, "On secrecy performance of miso swipt systems with tas and imperfect csi," IEEE Transactions on Communications, vol. 64, no. 9, pp. 3831-3843, 2016.

[138] Y. Yang, Q. Li, W.-K. Ma, J. Ge, and P. Ching, "Cooperative secure beamforming for af relay networks with multiple eavesdroppers," IEEE Signal Processing Letters, vol. 20, no. 1, pp. 35-38, 2013.

[139] H. Xing, L. Liu, and R. Zhang, "Secrecy wireless information and power transfer in fading wiretap channel," IEEE Transactions on Vehicular Technology, vol. 65, no. 1, pp. 180-190, 2016.

[140] Y. Liu, "Wireless information and power transfer for multirelay-assisted cooperative communication," IEEE Communications Letters, vol. 20 , no. 4, pp. 784-787, 2016.

[141] M. Marcus and B. Pattan, "Millimeter wave propagation: spectrum management implications," IEEE Microwave Magazine, vol. 6, no. 2 , pp. 54-62, 2005 .

[142] A. Al-Hourani, S. Chandrasekharan, and S. Kandeepan, "Path loss study for millimeter wave device-to-device communications in urban environment," in Communications Workshops (ICC), 2014 IEEE International Conference on. IEEE, 2014, pp. 102-107.

[143] J. Lillibridge, R. Scharroo, S. Abdalla, and D. Vandemark, "One-and two-dimensional wind speed models for ka-band altimetry," Journal of Atmospheric and Oceanic Technology, vol. 31, no. 3, pp. 630-638, 2014.

[144] L. Wang, M. Elkashlan, R. W. Heath, M. Di Renzo, and K.-K. Wong, "Millimeter wave power transfer and information transmission," in Global Communications Conference (GLOBECOM), 2015 IEEE. IEEE, 2015, pp. 1-6. 
[145] S. Ladan, A. B. Guntupalli, and K. Wu, "A high-efficiency 24 ghz rectenna development towards millimeter-wave energy harvesting and wireless power transmission," IEEE Transactions on Circuits and Systems I: Regular Papers, vol. 61, no. 12, pp. 3358-3366, 2014.

[146] A. Nesic, Z. Micic, S. Jovanovic, and I. Radnovic, "Millimeter wave printed antenna array with high side lobe suppression," in Antennas and Propagation Society International Symposium 2006, IEEE. IEEE, 2006, pp. 3051-3054.

[147] M. Peer, N. Jain, and V. A. Bohara, "A hybrid spectrum sharing protocol for energy harvesting wireless sensor nodes," in Signal Processing Advances in Wireless Communications (SPAWC), 2016 IEEE 17th International Workshop on. IEEE, 2016, pp. 1-6.

[148] I. F. Akyildiz, T. Melodia, and K. R. Chowdhury, "A survey on wireless multimedia sensor networks," Computer networks, vol. 51, no. 4, pp. 921-960, 2007.

[149] B. Tong, Z. Li, G. Wang, and W. Zhang, "How wireless power charging technology affects sensor network deployment and routing," in Distributed Computing Systems (ICDCS), 2010 IEEE 30th International Conference on. IEEE, 2010, pp. 438-447.

[150] Z. Popović, E. A. Falkenstein, D. Costinett, and R. Zane, "Low-power far-field wireless powering for wireless sensors," Proceedings of the IEEE, vol. 101, no. 6, pp. 1397-1409, 2013.

[151] S. Guo, C. Wang, and Y. Yang, "Mobile data gathering with wireless energy replenishment in rechargeable sensor networks," in INFOCOM, 2013 Proceedings IEEE. IEEE, 2013, pp. 1932-1940.

[152] S. Guo, Wang.U, and Y. Yang, "Joint mobile data gathering and energy provisioning in wireless rechargeable sensor networks," IEEE Transactions on Mobile Computing, vol. 13, no. 12, pp. 2836-2852, 2014.

[153] C. Wang, J. Li, F. Ye, and Y. Yang, "Netwrap: An ndn based realtimewireless recharging framework for wireless sensor networks," IEEE Transactions on Mobile Computing, vol. 13, no. 6, pp. 1283-1297, 2014

[154] S. Zhang, J. Wu, and S. Lu, "Collaborative mobile charging for sensor networks," in Mobile Adhoc and Sensor Systems (MASS), 2012 IEEE 9th International Conference on. IEEE, 2012, pp. 84-92.

[155] J. Johnson, E. Basha, and C. Detweiler, "Charge selection algorithms for maximizing sensor network life with uav-based limited wireless recharging," in Intelligent Sensors, Sensor Networks and Information Processing, 2013 IEEE Eighth International Conference on. IEEE, 2013, pp. 159-164.

[156] M. Y. Naderi, K. R. Chowdhury, S. Basagni, W. Heinzelman, S. De, and S. Jana, "Experimental study of concurrent data and wireless energy transfer for sensor networks," in Global Communications Conference (GLOBECOM), 2014 IEEE. IEEE, 2014, pp. 2543-2549.

[157] G. Amarasuriya and H. V. Poor, "Wireless information and power transfer in multi-way relay networks with massive mimo," in Global Communications Conference (GLOBECOM), 2015 IEEE. IEEE, 2015, pp. 1-7.

[158] P. Phunchongharn, E. Hossain, and D. I. Kim, "Resource allocation for device-to-device communications underlaying lte-advanced networks," IEEE Wireless Communications, vol. 20, no. 4, pp. 91-100, 2013.

[159] S. S. Nam, D. I. Kim, and H.-C. Yang, "Modified dynamic df for type2 ue relays," in Wireless Communications and Networking Conference (WCNC), 2012 IEEE. IEEE, 2012, pp. 1392-1397.

[160] H. H. Yang, J. Lee, and T. Q. Quek, "Green device-to-device communication with harvesting energy in cellular networks," in Wireless Communications and Signal Processing (WCSP), 2014 Sixth International Conference on. IEEE, 2014, pp. 1-6.

[161] Y. Liu, L. Wang, S. A. R. Zaidi, M. Elkashlan, and T. Q. Duong, "Secure d2d communication in large-scale cognitive cellular networks: A wireless power transfer model," IEEE Transactions on Communications, vol. 64, no. 1, pp. 329-342, 2016.

[162] S. Lee, R. Zhang, and K. Huang, "Opportunistic wireless energy harvesting in cognitive radio networks," IEEE Transactions on Wireless Communications, vol. 12, no. 9, pp. 4788-4799, 2013.

[163] Z. Zhang, K. Long, A. V. Vasilakos, and L. Hanzo, "Full-duplex wireless communications: challenges, solutions, and future research directions," Proceedings of the IEEE, vol. 104, no. 7, pp. 1369-1409, 2016.

[164] T. Riihonen and R. Wichman, "Energy detection in full-duplex cognitive radios under residual self-interference," in Cognitive Radio Oriented Wireless Networks and Communications (CROWNCOM), 2014 9th International Conference on. IEEE, 2014, pp. 57-60.

[165] G. Zheng, I. Krikidis, J. Li, A. P. Petropulu, and B. Ottersten, "Improving physical layer secrecy using full-duplex jamming receivers," IEEE
Transactions on Signal Processing, vol. 61, no. 20, pp. 4962-4974, 2013.

[166] S. K. Sharma, T. E. Bogale, L. B. Le, S. Chatzinotas, X. Wang, and B. Ottersten, "Two-phase concurrent sensing and transmission scheme for full duplex cognitive radio," in Vehicular Technology Conference (VTC-Fall), 2016 IEEE 84th. IEEE, 2016, pp. 1-5.

[167] S. Hu, Z. Ding, and Q. Ni, "Beamforming optimisation in energy harvesting cooperative full-duplex networks with self-energy recycling protocol," IET Communications, vol. 10, no. 7, pp. 848-853, 2016.

[168] Z. Wen, X. Liu, N. C. Beaulieu, R. Wang, and S. Wang, "Joint source and relay beamforming design for full-duplex mimo af relay swipt systems," IEEE Communications Letters, vol. 20, no. 2, pp. 320-323, 2016.

[169] D. N. Jayakody and M. F. Flanagan, "A soft decode-compress-forward relaying scheme for cooperative wireless networks," IEEE Transactions on Vehicular Technology, vol. 65, no. 5, pp. 3033-3041, 2016.

[170] D. N. Jayakody, J. Li, and M. F. Flanagan, "A soft-network-coded multilevel forwarding scheme for multiple-access relay systems," IEEE Transactions on Vehicular Technology, vol. 65, no. 5, pp. 3430-3439, 2016.

[171] O. Taghizadeh, A. Zamani, and R. Mathar, "Physical-layer security for simultaneous information and power transfer in full-duplex multi-user networks," in Smart Antennas (WSA 2016); Proceedings of the 20th International ITG Workshop on. VDE, 2016, pp. 1-8.

[172] F. Zhu, F. Gao, and M. Yao, "Zero-forcing beamforming for physical layer security of energy harvesting wireless communications," EURASIP Journal on Wireless Communications and Networking, vol. 2015, no. 1, p. 58, 2015.

[173] A. Salem, K. A. Hamdi, and K. M. Rabie, "Physical layer security with rf energy harvesting in af multi-antenna relaying networks," IEEE Transactions on Communications, vol. 64, no. 7, pp. 3025-3038, 2016.

[174] L. Liu, R. Zhang, and K.-C. Chua, "Secrecy wireless information and power transfer with miso beamforming," in Global Communications Conference (GLOBECOM), 2013 IEEE. IEEE, 2013, pp. 1831-1836.

[175] S. Guo, F. Wang, Y. Yang, and B. Xiao, "Energy-efficient cooperative tfor simultaneous wireless information and power transfer in clustered wireless sensor networks," IEEE Transactions on Communications, vol. 63, no. 11, pp. 4405-4417, 2015.

[176] Q. Shi, W. Xu, J. Wu, E. Song, and Y. Wang, "Secure beamforming for mimo broadcasting with wireless information and power transfer," IEEE Transactions on Wireless Communications, vol. 14, no. 5, pp. 2841-2853, 2015.

[177] E. Boshkovska, R. Morsi, D. W. K. Ng, and R. Schober, "Power allocation and scheduling for swipt systems with non-linear energy harvesting model," Communications (ICC), IEEE International Conference, pp. $1-6,2016$.

[178] D. K. Nguyen, D. N. Jayakody, S. Chatzinotas, J. Thompson, and J. Li, "Wireless energy harvesting assisted two-way cognitive relay networks: Protocol design and performance analysis," IEEE Access, 2017.

[179] S. Q. Nguyen, H. Y. Kong et al., "Performance analysis of energyharvesting relay selection systems with multiple antennas in presence of transmit hardware impairments," in Advanced Technologies for Communications (ATC), 2016 International Conference on. IEEE, 2016, pp. 126-130.

[180] I. Tal and A. Vardy, "How to construct polar codes," IEEE Transactions on Information Theory, vol. 59, no. 10, pp. 6562-6582, 2013.

[181] H. Kong, C. Xing, S. Zhao, and P. Shi, "Cooperative coding scheme using polar codes," in Computer Science and Network Technology (ICCSNT), 2012 2nd International Conference on. IEEE, 2012, pp. 602-606.

[182] B. Yuan and K. K. Parhi, "Early stopping criteria for energy-efficient low-latency belief-propagation polar code decoders," IEEE Transactions on Signal Processing, vol. 62, no. 24, pp. 6496-6506, 2014.

[183] R. Blasco-Serrano, R. Thobaben, M. Andersson, V. Rathi, and M. Skoglund, "Polar codes for cooperative relaying," IEEE Transactions on Communications, vol. 60, no. 11, pp. 3263-3273, 2012.

[184] K. He, J. Sha, L. Li, and Z. Wang, "Low power decoder design for qc-ldpc codes," in Circuits and Systems (ISCAS), Proceedings of 2010 IEEE International Symposium on. IEEE, 2010, pp. 3937-3940.

[185] Y. S. Park, D. Blaauw, D. Sylvester, and Z. Zhang, "Low-power highthroughput ldpc decoder using non-refresh embedded dram," IEEE Journal of Solid-State Circuits, vol. 49, no. 3, pp. 783-794, 2014.

[186] J. Kaza and C. Chakrabarti, "Design and implementation of low-energy turbo decoders," IEEE Transactions on Very Large Scale Integration (VLSI) Systems, vol. 12, no. 9, pp. 968-977, 2004.

[187] R. G. Maunder, "The 5g channel code contenders," AccelerComm White Paper, pp. 1-13, 2016. 
[188] S. K. Sharma, T. E. Bogale, S. Chatzinotas, X. Wang, and L. B. Le, "Physical layer aspects of wireless iot," in Wireless Communication Systems (ISWCS), 2016 International Symposium on. IEEE, 2016, pp. 304-308.

[189] G. Reiter, "Wireless connectivity for the internet of things," Europe, vol. 433, p. $868 \mathrm{MHz}, 2014$.

[190] S. Zhou, T. Chen, W. Chen, and Z. Niu, "Outage minimization for a fading wireless link with energy harvesting transmitter and receiver," IEEE Journal on Selected Areas in Communications, vol. 33, no. 3, pp. 496-511, 2015.

[191] B. W. Khoueiry and M. R. Soleymani, "A novel machine to machine communication strategy using rateless coding for the internet of things," in Beyond the Internet of Things. Springer, 2017, pp. 85-118.

[192] H. Matsumoto and K. Hashimoto, "Report of the ursi inter-commission working group on sps and appendices," in White Paper on Solar Power Satellite (SPS) Systems for the General Assembly and Scientific Symp. of Int. Union of Radio Science; http://www. ursi. org 6 , URSI, Ghent, Belgium, 2006. 